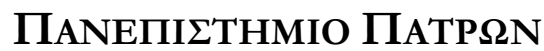

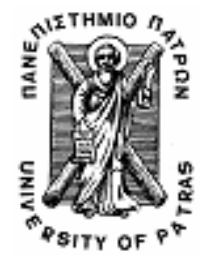

TMHMA IATPIKH $\Sigma$

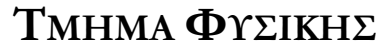

DIATMHМАTIKO ПРОГРАММА

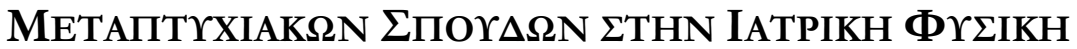

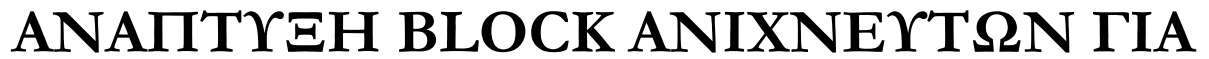 TOMОГРАФО ЕКПОМПНГ ПОZITPONISN (PET)}

\author{
MAPIA-E $\Lambda E N H ~ N I K O \Lambda A O Y$

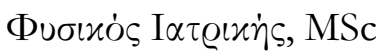

$\Delta \mathrm{I} \Delta$ AKTOPIKH $\triangle \mathrm{IATPIBH}$

ПАТРА 2008 



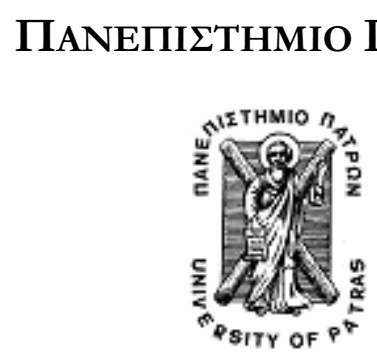

TMHMA IATPIKH $\Sigma$

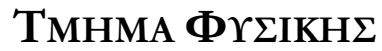

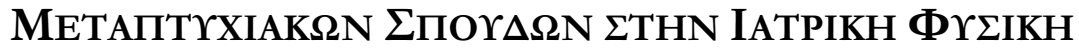

\section{ANAחTYEH BLOCK ANIXNEYTSN ГIA

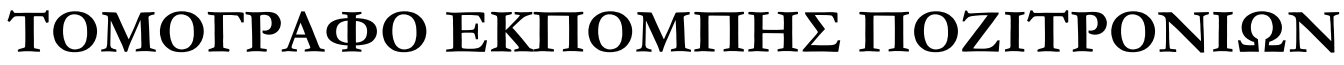 (PET)}
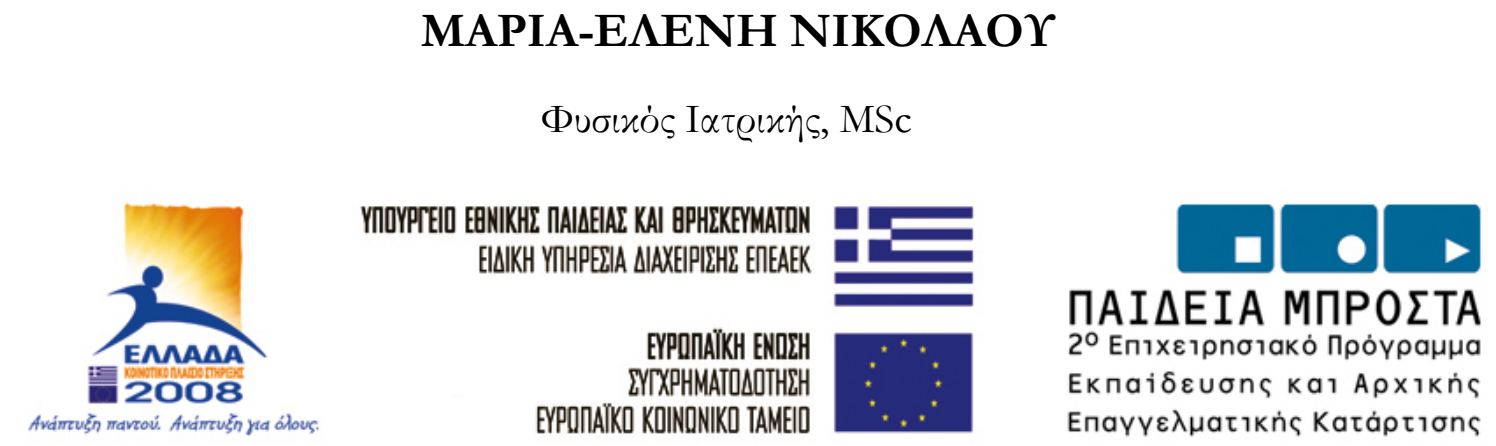

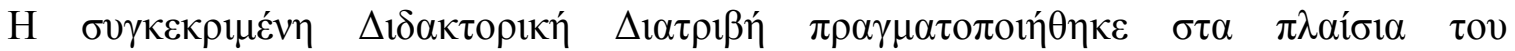

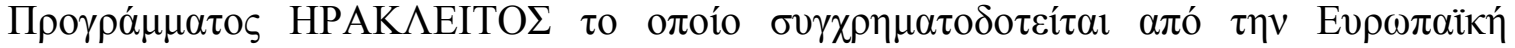

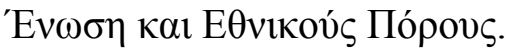

\section{$\Delta \mathrm{I} \Delta$ AKTOPIKH $\Delta \mathrm{IATPIBH}$}

ПАТРА 2008 

University OF Patras

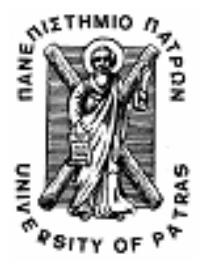

SCHOOL OF MEDICINE

Department of Physics

\author{
INTERDEPARTMENTAL Program
}

of Postgraduate Studies in Medical Physics

\title{
DEVELOPMENT OF BLOCK DETECTORS
}

\section{FOR POSITRON EMISSION TOMOGRAPHY (PET)}

MARIA-ELENI NIKOLAOU

Medical Physicist, MSc

PHILOSOPHY DOCTORATE THESIS

PATRAS, 2008 



\section{THE THREE MEMBER ADVISORY COMMITTEE:}

Georgios Panayiotakis, Professor

Georgios Tzanakos, Professor

Pavlos Vassilakos, Professor
Supervisor

Member of the Advisory Committee

Member of the AdvisoryCommittee

\section{THE SEVEN MEMBER EXAMINATION COMMITTEE:}

Georgios Panayiotakis, Professor

Georgios Tzanakos, Professor

Pavlos Vassilakos, Professor

Georgios Nikiforidis, Professor

Nikolaos Pallikarakis, Professor

Dimitrios Apostolopoulos, Assistant Professor

Eleni Costaridou, Assistant Professor
Supervisor

Member of the Examination Committee Member of the Examination Committee Member of the Examination Committee Member of the Examination Committee Member of the Examination Committee Member of the Examination Committee 


\section{Acknowledgements}

I would like to thank Professor Georgios Panayiotakis, Professor Georgios Tzanakos and Professor Pavlos Vassilakos for supervising my thesis.

Especially, I would like to express my deepest gratitude to Professor Georgios Panayiotakis for everything that he did for me during these years, for his support, patience, advice and guidance. I feel honored to be one of his students.

This thesis wouldn't have come to an end without the continuous support from my husband, Panayotis. He really believed in me and was always there to support me in hard times, to give me some good advice whenever I needed it, assist me in many scientific issues and take care of our two daughters, even though he had to set aside his own obligations, while I was working on my thesis, among all other things. I'm really grateful to God for his presence in my life. Thank you for everything that you do and you are for me... Our two daughters, Despina and Athena, although very young and unaware of what their 'mama' was doing all this time, were/are always a source of joy with their smiles, laughs, mumbling, kisses and hugs. Even though some of my choices were very difficult for them, it was very important for me to know that they would be beside me....

Also, I would like to thank Professor Georgios Tzanakos for giving me the opportunity to work in the HEPA laboratory. In this laboratory I met two good friends and collaborators, Evagelos Logaras and Miltiadis Zois. Thank you very much for all your help, 
for being there in good and hard times, and for all the laughs we had together. It was really 'refreshing' having you around.

Many thanks to Dr. George Spyrou for all his help and support from the days I was an undergraduate student, until now that this doctorate thesis is completed. His own scientific trajectory was inspiring for me.

My sincere thanks to Dr. Spyros Skiadopoulos for being always available whenever I asked for his help, for his patience and for his prompt response.

Last but not least, I would like to thank my parents, Georgios and Athena, my beloved sisters Kiki, Olympia and Dina and their families, my husband's parents, my cousin Christina, my aunt Stathoula and all my friends for their love, caring, help and support. 


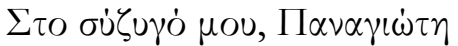

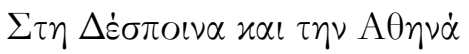




\section{Table of Contents}

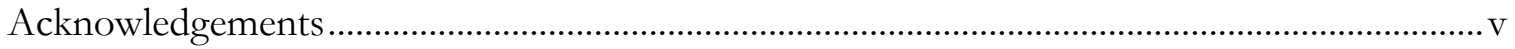

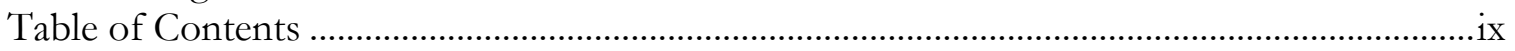

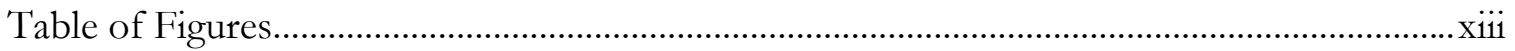

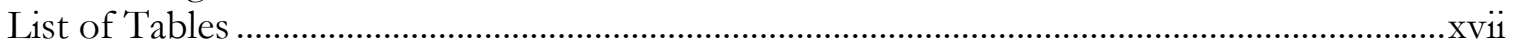

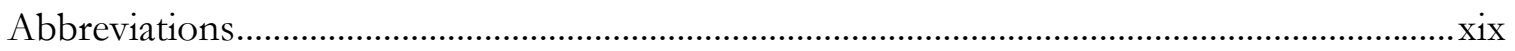

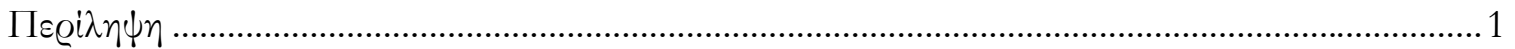

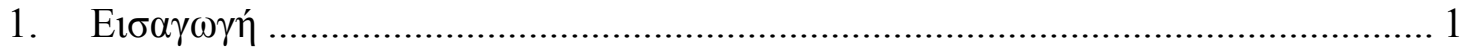

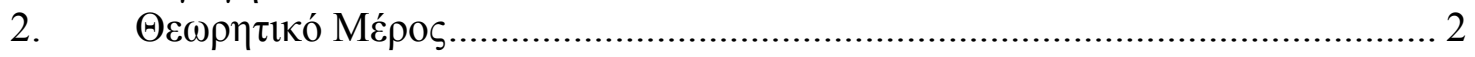

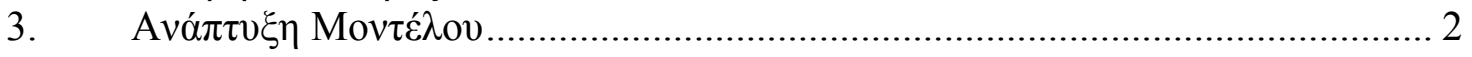

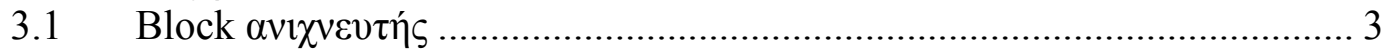

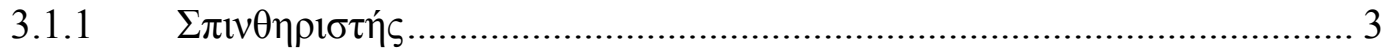

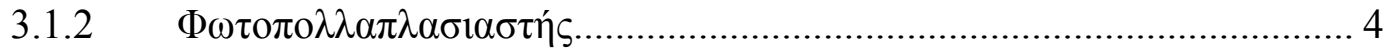

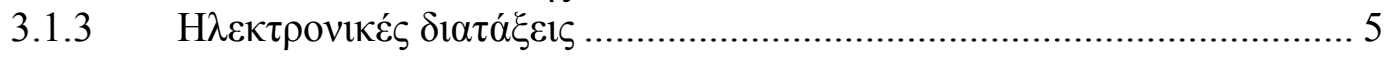

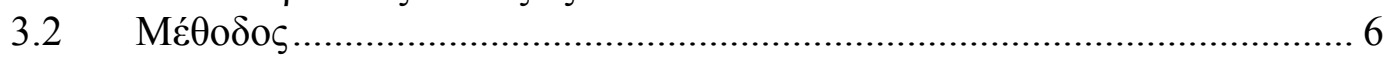

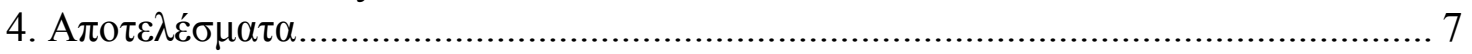

4.1 Ev

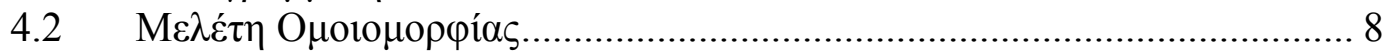

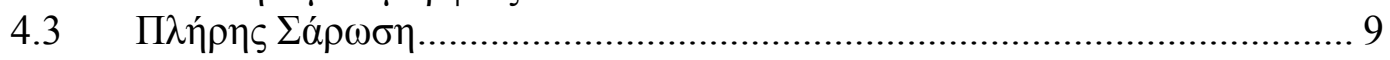

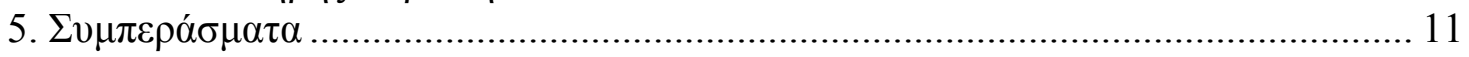

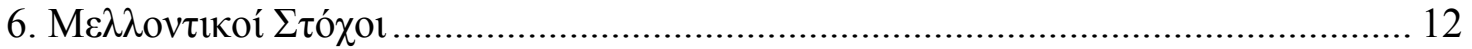

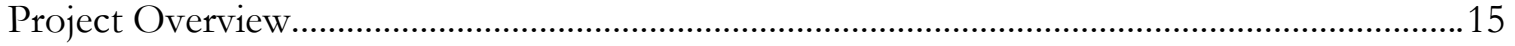

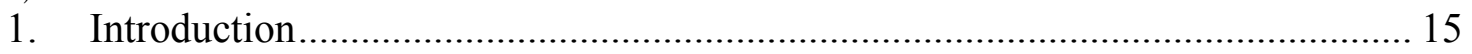

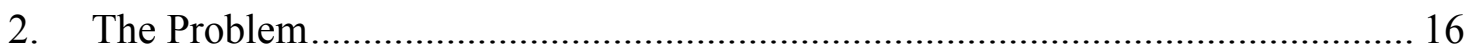

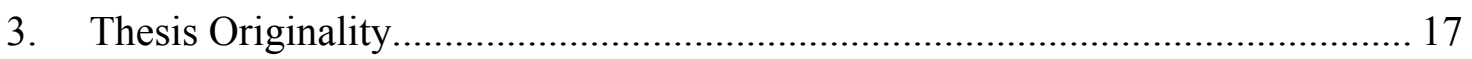

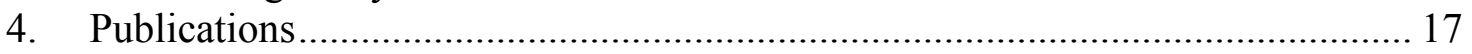

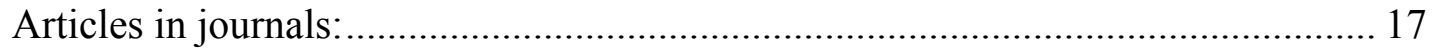

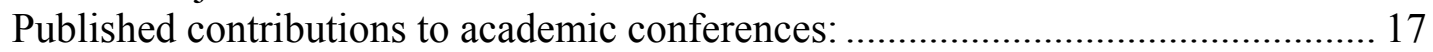

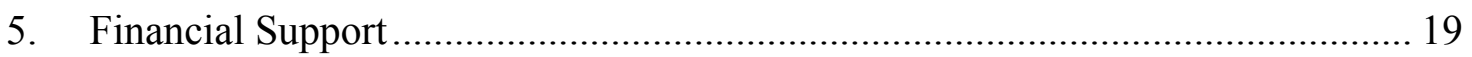

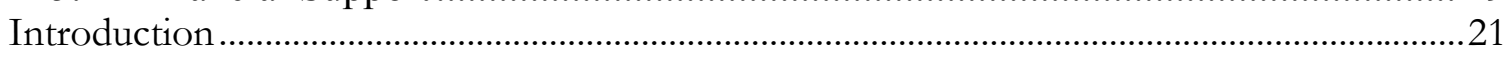

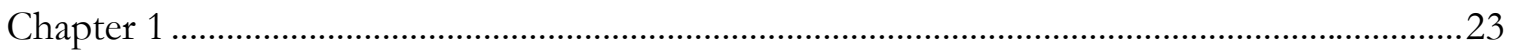




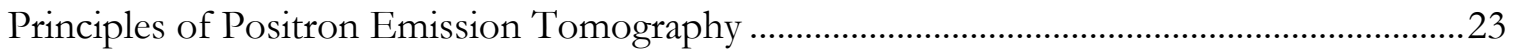

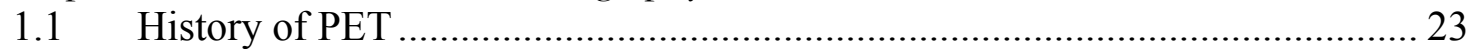

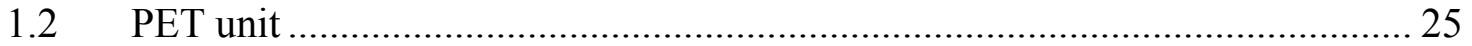

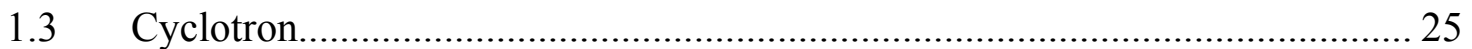

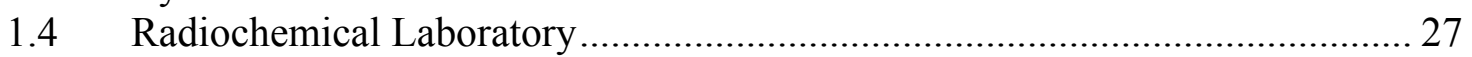

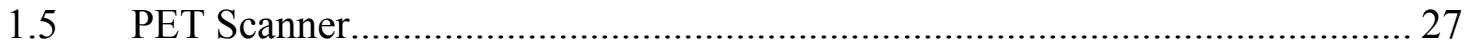

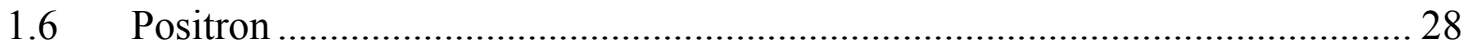

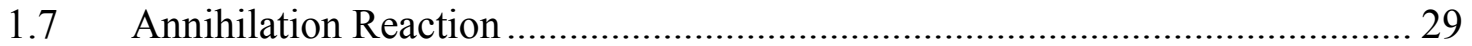

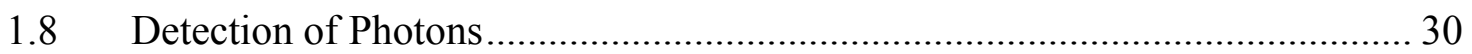

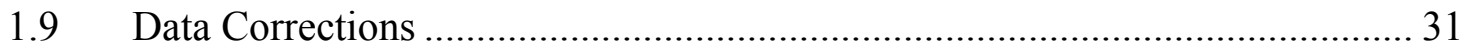

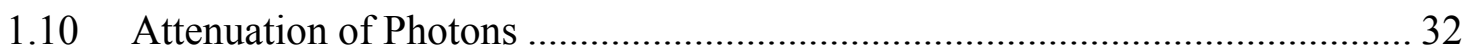

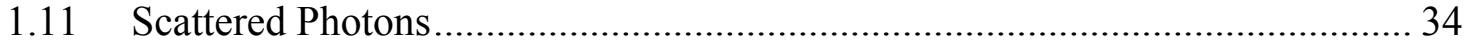

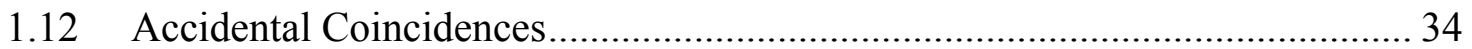

1.13 Image Reconstruction ............................................................................ 35

1.14 Clinical Applications of PET ................................................................... 36

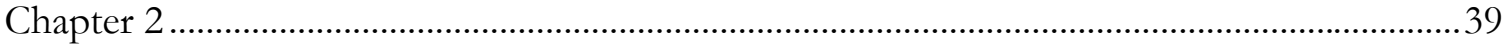

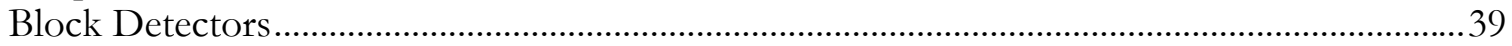

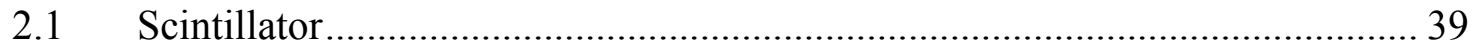

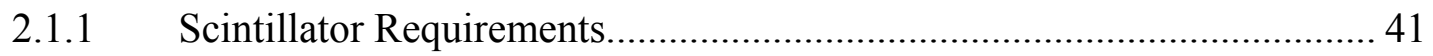

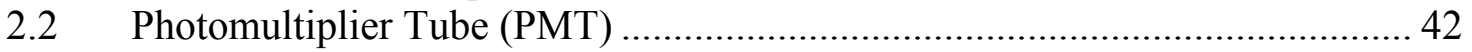

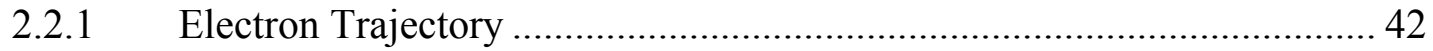

2.2.2 Characteristics of Photomultiplier Tubes............................................. 44

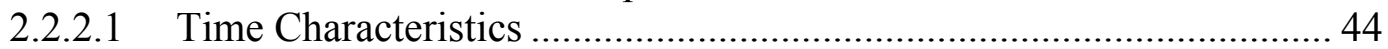

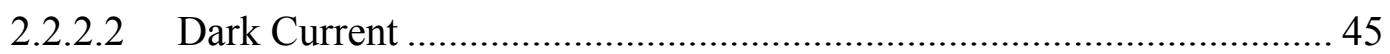

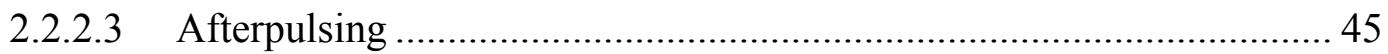

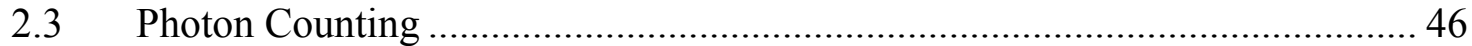

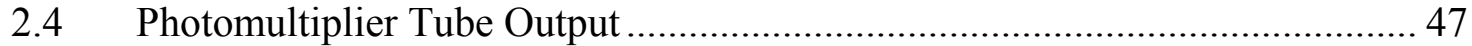

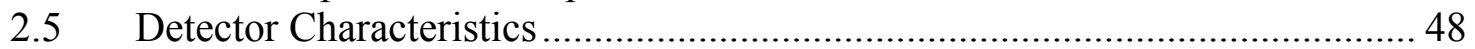

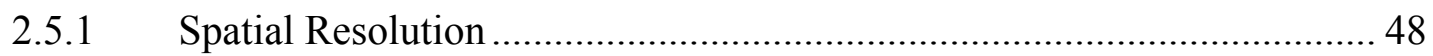

2.5.2 Energy Resolution........................................................................... 49

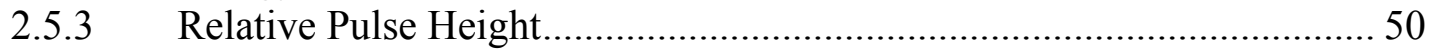

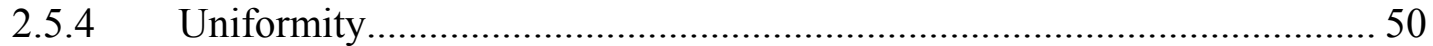

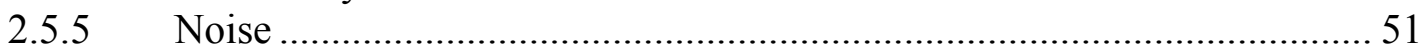

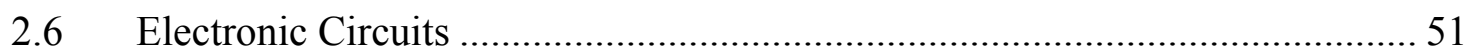

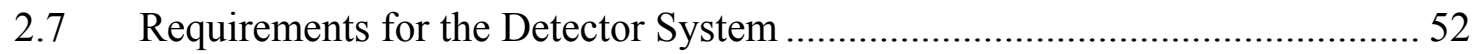

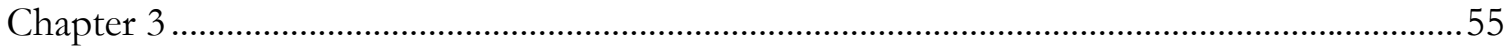

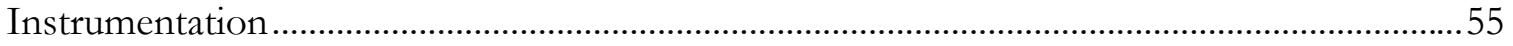

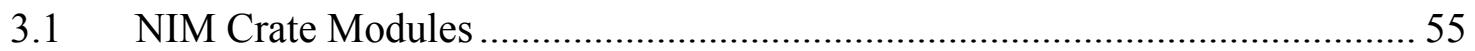

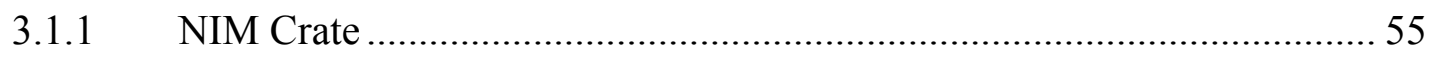

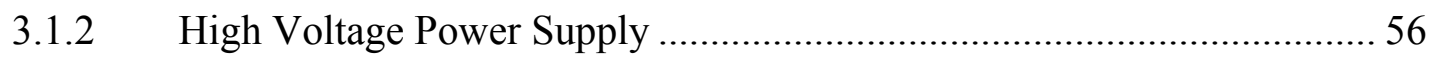

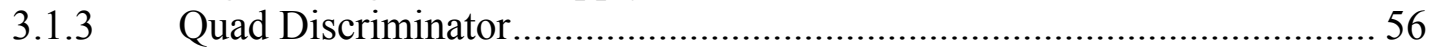

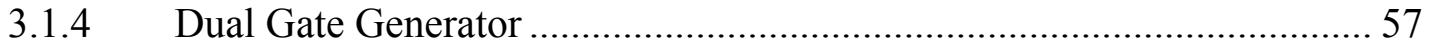

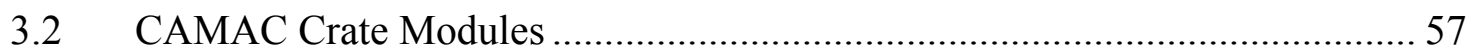

3.2.1 CAMAC Crate ................................................................................... 57

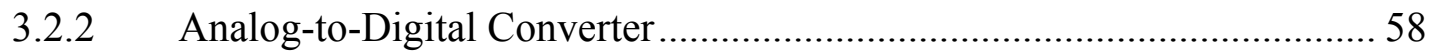

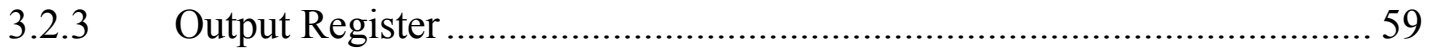

3.2.4 Crate Controller ...................................................................................... 59 


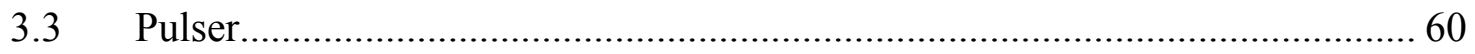

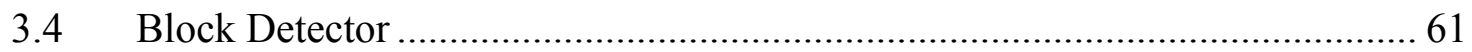

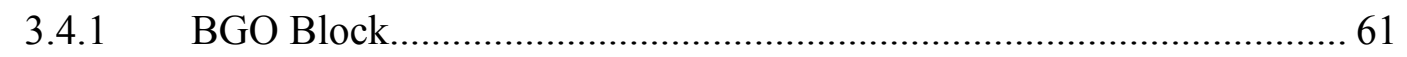

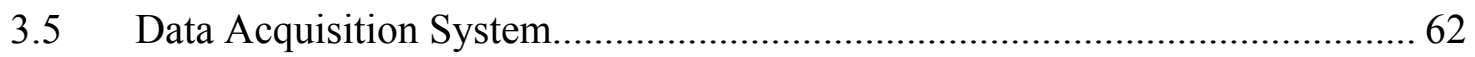

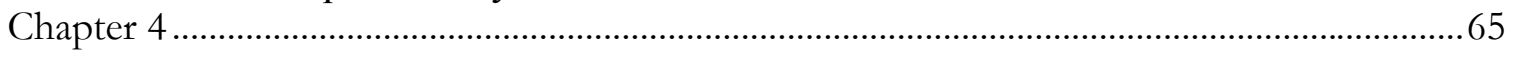

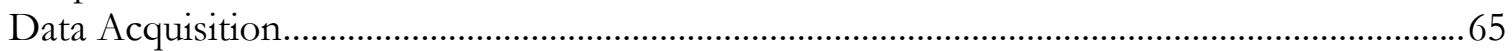

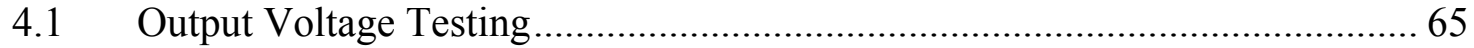

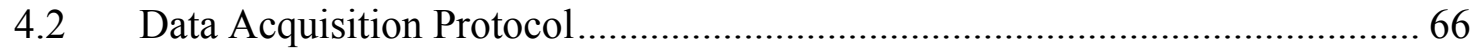

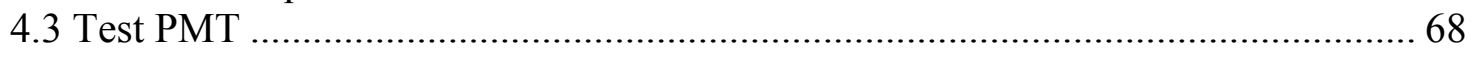

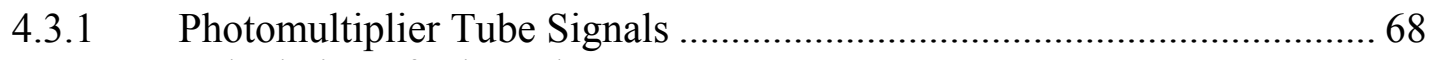

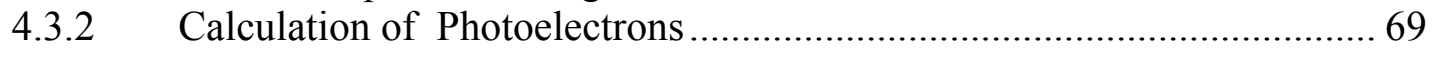

4.3.3 Gain Calculation ............................................................................... 71

4.3.4 Dependence of ADC Counts on High Voltage ......................................... 71

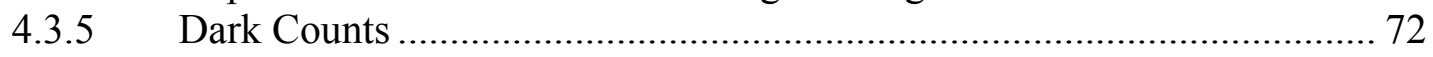

4.4 System Photomultiplier Tubes Study ……………....................................... 73

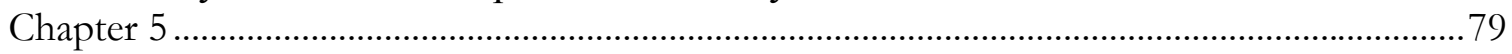

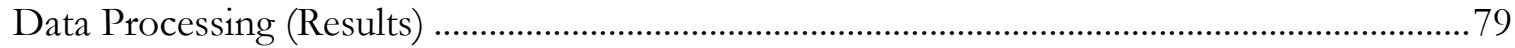

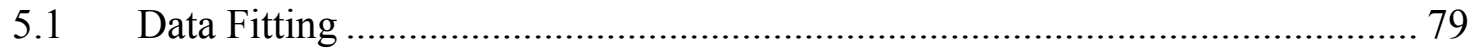

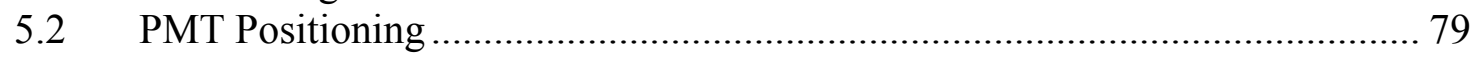

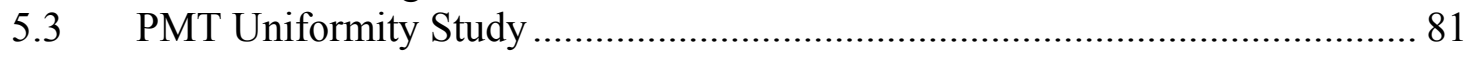

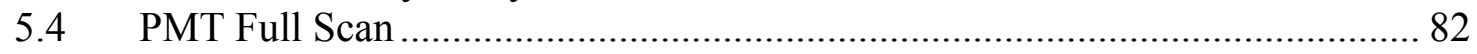

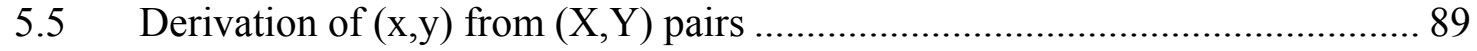

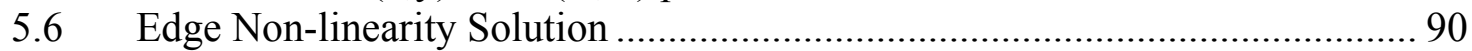

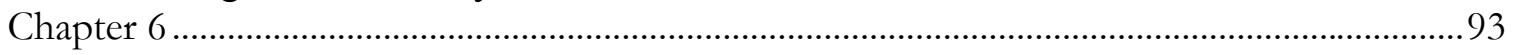

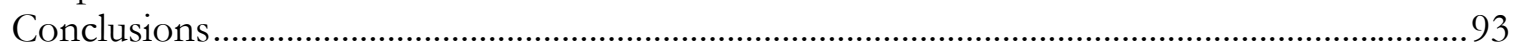

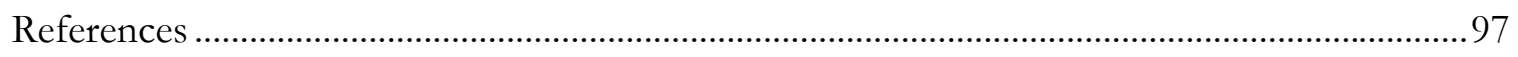




\section{Table of Figures}

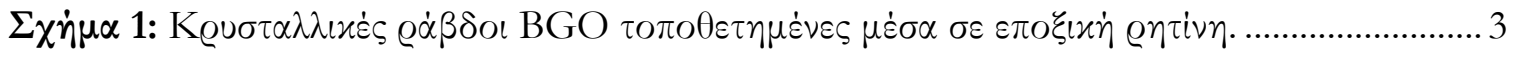

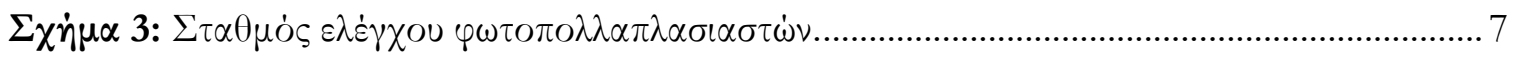

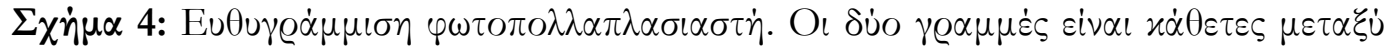

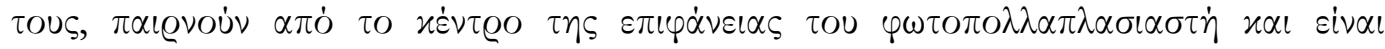

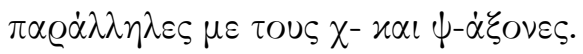

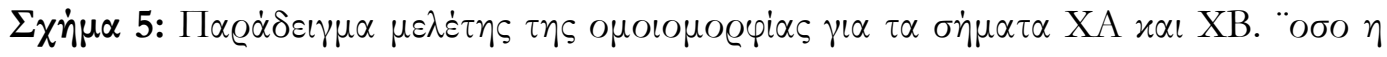

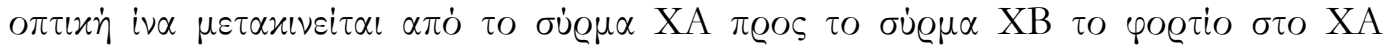

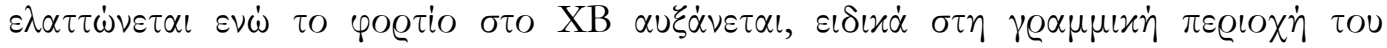
$\varphi \omega \tau 0 \pi \circ \lambda \lambda \alpha \pi \lambda \alpha \sigma \iota \alpha \sigma \tau \dot{\eta}$

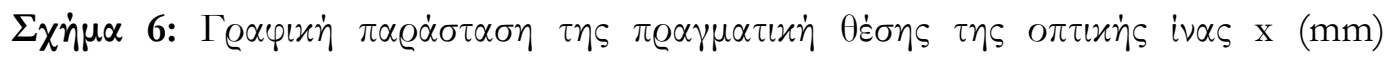

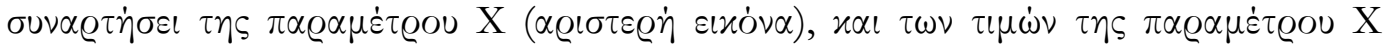

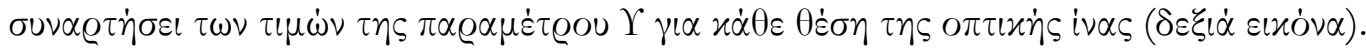

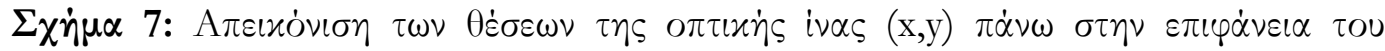
$\varphi \omega \tau O \pi \circ \lambda \lambda \alpha \pi \lambda \alpha \sigma \iota \alpha \sigma \tau \dot{\eta}$

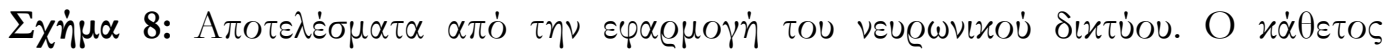

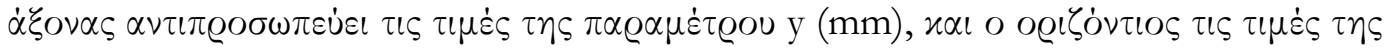

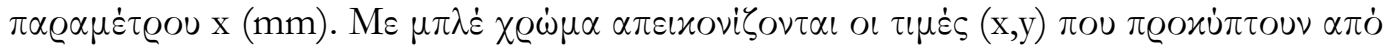

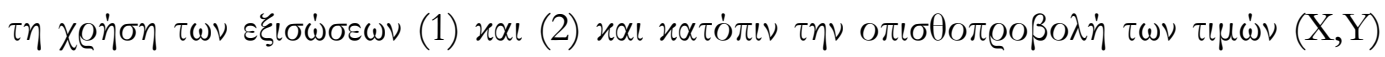




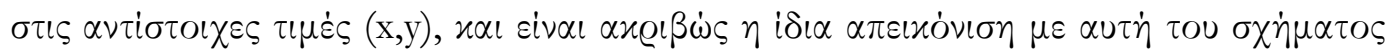

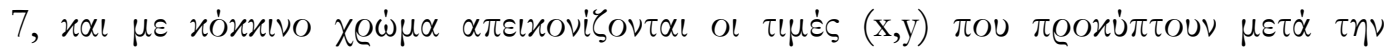

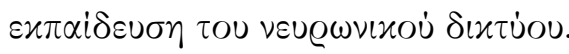

Figure 1.1: Positron emission followed by an annihilation reaction. The annihilation reaction is shown to the right in a Feynman diagram with space-time vectors.

Figure 1.2: Coincidence detection in an attenuating object. 33

Figure 1.3: Diagram of a true event, a scattered event and an accidental coincidence.

The dots represent the annihilation event; the arrows represent the gamma rays, and the dashed lines show the corresponding lines of response.

Figure 2.1: Cross-section of a head-on type photomultiplier tube. [Hamamatsu] ...................42

Figure 2.2: Schematic of rise, fall and electron transit time.................................................... 44

Figure 2.3: Gamma-ray absorption characteristics of $\mathrm{NaI}(\mathrm{Tl})$ scintillator. ............................... 46

Figure 2.4: Gamma-ray detection using a scintillator and a photomultiplier tube. ................ 47

Figure 2.3: Definition of the energy resolution using the pulse height spectrum...................49

Figure 3.1: BGO crystal boules encapsulated in epoxy resin.

Figure 3.2: Data Acquisition system photographs. The first photograph shows the NIM and CAMAC crates along with their modules, and also the digital oscilloscope for the display of the output signals. The second photograph shows the DAQ and the analysis personal computers and also the dark box where the photomultiplier tubes are placed for testing......

Figure 3.3: Schematic diagram of the data acquisition system

Figure 4.1: Plot of the polymeter indications as a function of the high voltage power supply indications. As it can be noticed the two indications are linearly related.......

Figure 4.2: Photomultiplier tube output pulse height as a function of the applied high voltage.

Figure 4.3: Discriminator output signal displayed together with the photomultiplier tube signal.

Figure 4.4: Photomultiplier tube output signal (above) and its corresponding pedestal (below).

Figure 4.5: ADC counts (the ADC counts of the signal have been subtracted from the sPMT signal ADC counts) as a function of the applied high voltage. 72

Figure 4.6: Dark counts measurement with a preset HV of $1500 \mathrm{~V}$. 
Figure 4.7: The Hamamatsu R2486-05 SELECT series photomultiplier tube. 74

Figure 4.8: Crossed-wire anode construction......

Figure 4.9: Dark box contents. The photomultiplier tube, $x-y$ stage system, and the optical fiber that extends to the photomultiplier tube surface.

Figure 5.1: X- and Y-Axis of R2486 PMT.

Figure 5.2: PMT A cross-like scan to determine the positioning accuracy.

Figure 5.3: PMT B cross-like scan to determine the positioning accuracy.

Figure 5.4: Digitized signals $\mathrm{XA}$ and $\mathrm{XB}$, and $\mathrm{XA}+\mathrm{XB}$ (with subtracted pedestals)

plotted versus the real position of the optical fiber along $\mathrm{x}$-axis.

Figure 5.5: Positioning variable $\mathrm{X}$ versus the true fiber position $\mathrm{x}$ for PMT A

Figure 5.6: Positioning variable $X$ versus the true fiber position $x$ for PMT B.

Figure 5.7: $\mathrm{X}$ versus $\mathrm{Y}$ plot in a full PMT A scan.

Figure 5.8: $\mathrm{X}$ versus $\mathrm{Y}$ plot in a full PMT A scan. In this case, several points have been omitted using specific criteria concerning the $\chi$-square, the number of degrees of freedom and the mean value of each digitized spectrum. 86

Figure 5.9: $\mathrm{X}$ versus $\mathrm{Y}$ plot in a full PMT B scan.

Figure 5.10: $\mathrm{X}$ versus $\mathrm{Y}$ plot in a full PMT B scan. In this case, several points have been omitted using specific criteria concerning the $\chi$-square, the number of degrees of freedom and the mean value of each digitized spectrum. 87

Figure 5.11: True positions $(\mathrm{x}, \mathrm{y})$ of the optical fiber derived by the calculated $(\mathrm{X}, \mathrm{Y})$ pairs for PMT A.

Figure 5.12: True positions $(\mathrm{x}, \mathrm{y})$ of the optical fiber derived by the calculated $(\mathrm{X}, \mathrm{Y})$ pairs for PMT B.

Figure 5.13: 2-dimensional image obtained before and after the application of a neural network on the data points $(\mathrm{x}, \mathrm{y})$. The vertical axis represents the values of the parameter $y$, while the horizontal axis represents the values of the parameter $\mathrm{x}$. The blue points are the ones calculated by the digitized signals $\mathrm{XA}, \mathrm{XB}, \mathrm{YC}$ and $\mathrm{YD}$ with backprojection to the actual optical fiber position $(x, y)$, while the red points correspond to the points $(\mathrm{x}, \mathrm{y})$ resulting from the trained neural network. 


\section{List of Tables}

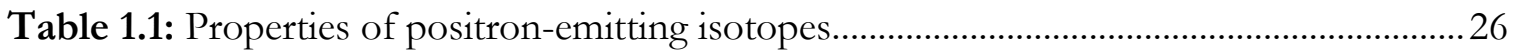

Table 2.1: Properties of the most frequently used inorganic scintillators. ................................ 40

Table 3.1: Specification data for N471 high voltage power supply..........................................56

Table 3.2: CAMAC Model 2249W Analog-to-Digital Converter specifications...................... 58

Table 4.1: Characteristics of PMT A (Hamamatsu R2486-05 Serial No NA0420)..................75

Table 4.2: Characteristics of PMT B (Hamamatsu R2486-05 Serial No NA0406)................. 75 
xviii 


\section{Abbreviations}

$\begin{array}{ll}\text { ACB } & \text { Auxiliary Control Bus } \\ \text { ADC } & \text { Analog-to-Digital Converter } \\ \text { AEN } & \text { Asynchronous Event Notification } \\ \mathrm{BaF}_{2} & \text { Barium Fluoride } \\ \text { BGO } & \text { Bi }_{4} \mathrm{Ge}_{3} \mathrm{O}_{12}-\text { Bismuth Germanate Oxide } \\ \text { C } & \text { Carbon } \\ \text { CAMAC } & \text { Computer Automated Measurement and Control } \\ \text { CT } & \text { Computerized Tomography } \\ \text { DAQ } & \text { Data Acquisition } \\ \text { ISA } & \text { Industry Standard Architecture } \\ \text { F } & \text { Fluoride } \\ \text { FOV } & \text { Field-Of-View } \\ \text { FPGA } & \text { Field Programmable Gate Array } \\ \text { FWHM } & \text { Full Width at Half Maximum } \\ \text { GSO } & \text { Gd }{ }_{2} \text { SiO }{ }_{5}-\text { Gadolinium Orthosilicate } \\ \text { LAM } & \text { Look-At-Me }\end{array}$




\begin{tabular}{|c|c|}
\hline LED & Light Emitting Diode \\
\hline LLD & Lower energy-level Discriminator \\
\hline LSO & $\mathrm{Lu}_{2} \mathrm{SiO}_{5}$ - Lutetium oxyorthosilicate \\
\hline LOR & Line-Of-Response \\
\hline $\mathrm{N}$ & Nitrogen \\
\hline $\mathrm{NaI}(\mathrm{Tl})$ & Thallium activated Sodium Iodide \\
\hline NIM & Nuclear Instrument Module \\
\hline $\mathrm{O}$ & Oxygen \\
\hline PC & Personal Computer \\
\hline PET & Positron Emission Tomography \\
\hline PHA & Pulse Height Analyzer \\
\hline PLD & Programmable Logic Device \\
\hline PMT & Photomultiplier Tube \\
\hline PSPMT & Position-Sensitive Photomultiplier Tube \\
\hline $\mathrm{RPH}$ & Relative Pulse Height \\
\hline SCSI & Small Computer System Interface \\
\hline TOF & Time-Of-Flight \\
\hline T'TL & Transistor-Transistor Logic \\
\hline TTS & Transit Time Spread \\
\hline ULD & Upper energy-level Discriminator \\
\hline Z & Atomic Number \\
\hline
\end{tabular}




\section{$\Pi \varepsilon \varrho i \lambda \eta \psi \eta$}

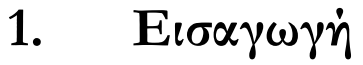

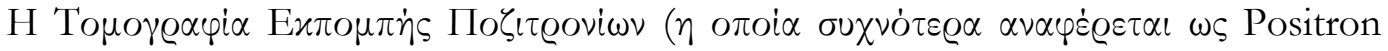

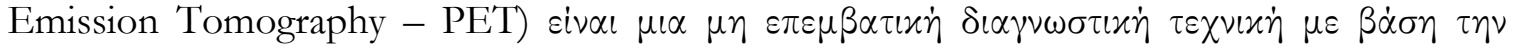

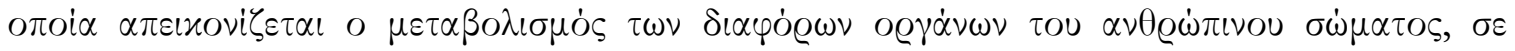

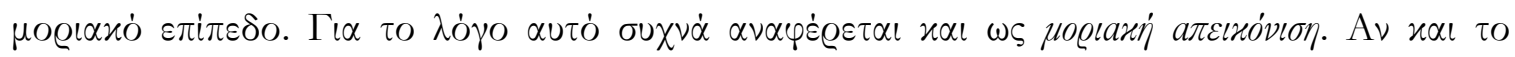

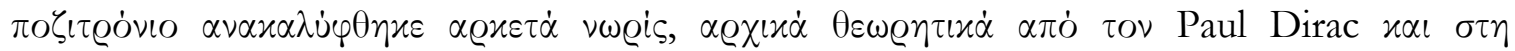

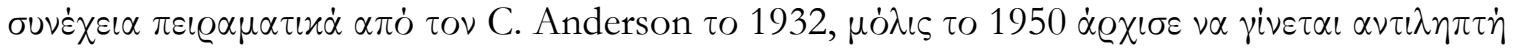

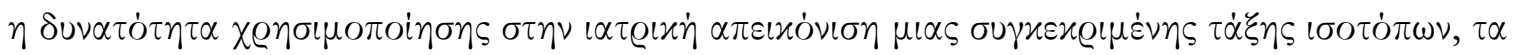

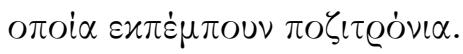

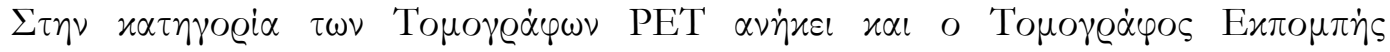

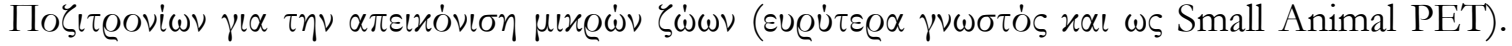

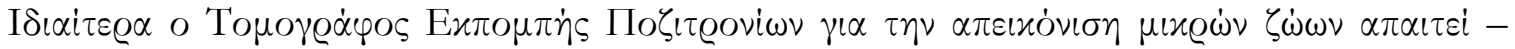

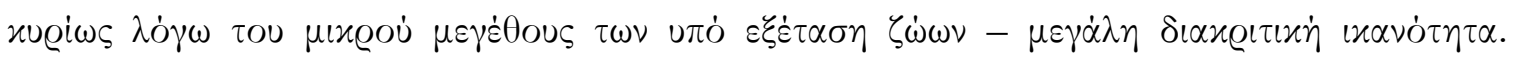

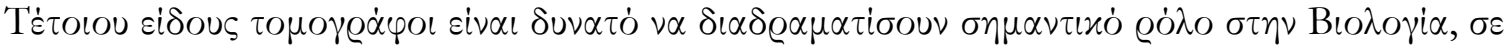

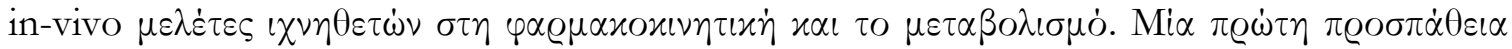




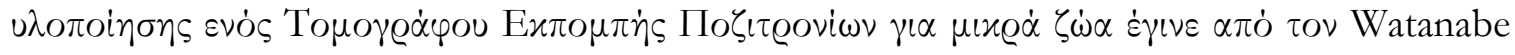

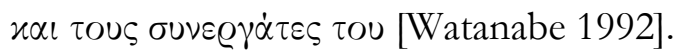

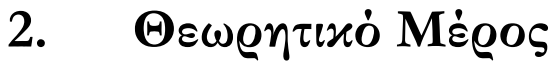

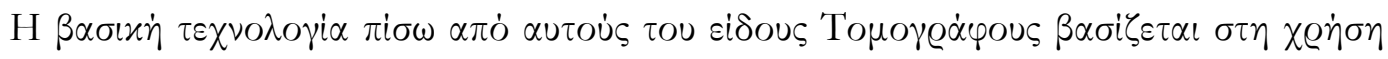

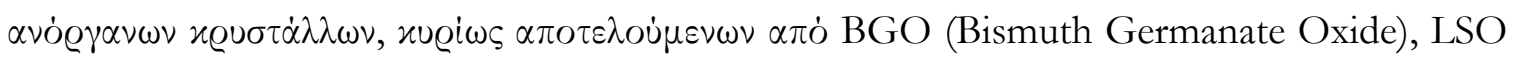
(Lutetium Oxyorthosilicate) $x \propto \iota$ GSO (Gadolimuim Oxyorthosilicate), o о олоio

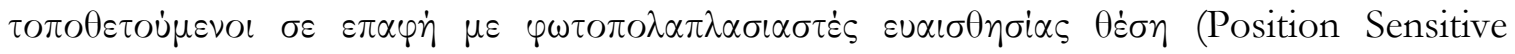

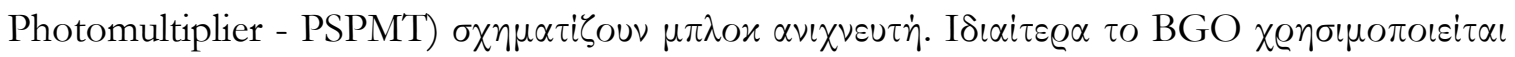

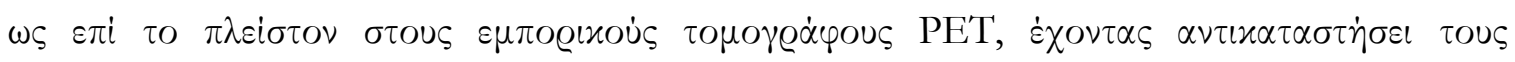

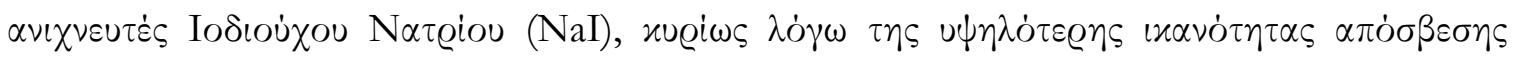

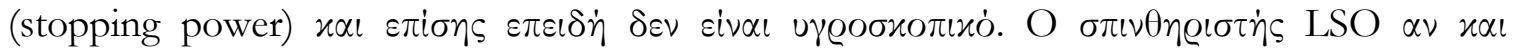

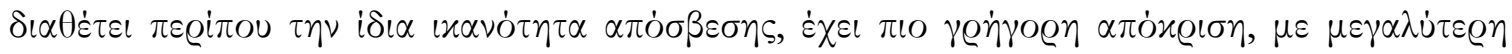

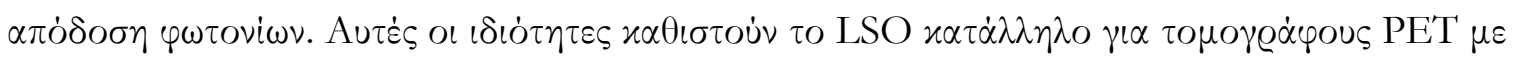

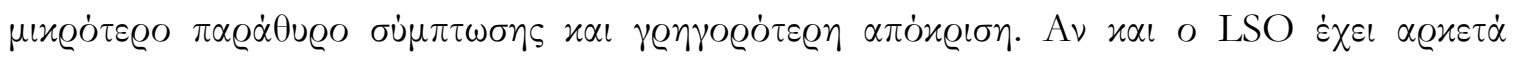

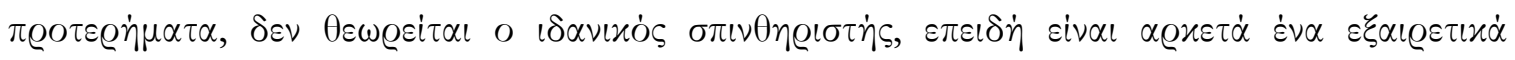

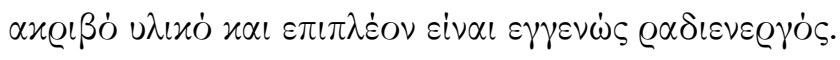

\section{A $\quad \dot{\alpha} \pi \tau v \xi \eta \eta$}

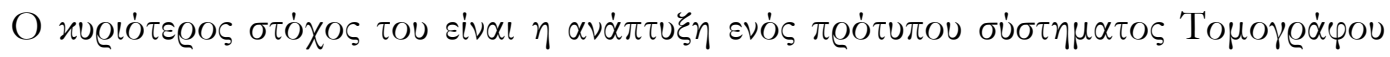

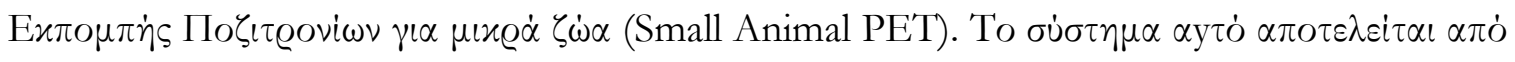

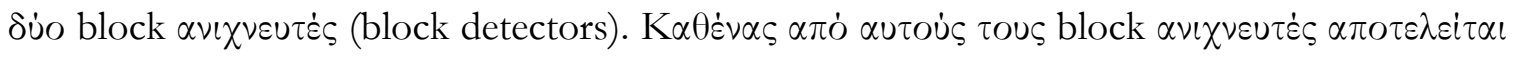

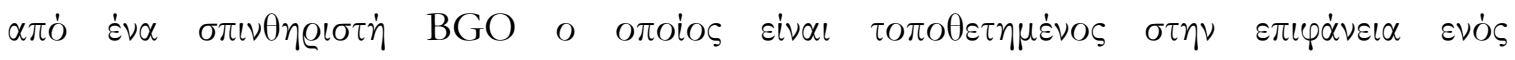

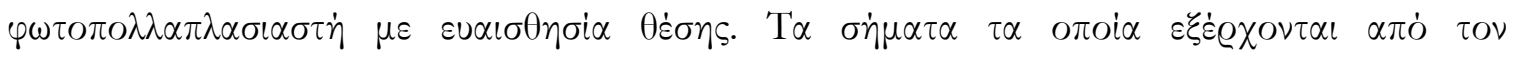

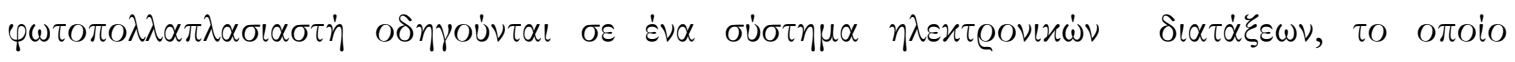

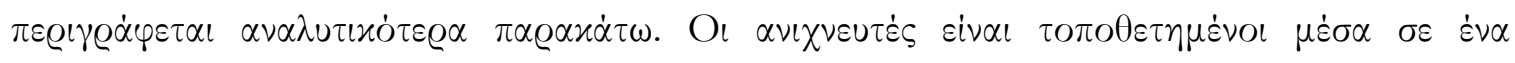

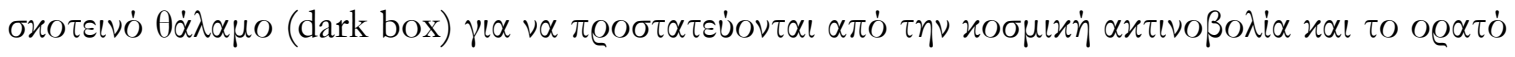
$\varphi \omega \varsigma$. 


\subsection{Block $\alpha \nu \iota \chi \nu \varepsilon u \tau \dot{\eta} s$}

\subsection{1 $\Sigma \pi \iota \nu \theta \eta \varrho \iota \tau \dot{\eta} \varsigma$}

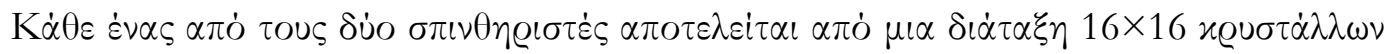

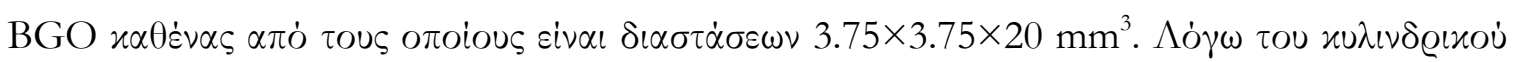

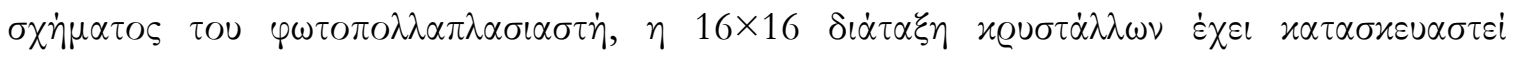

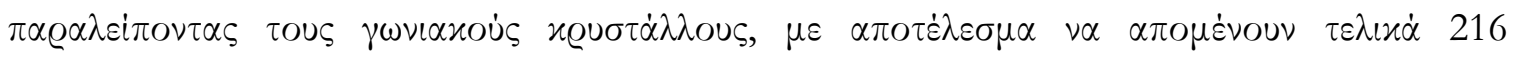

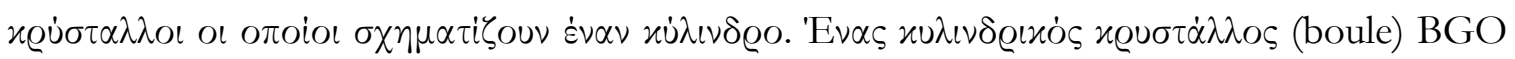

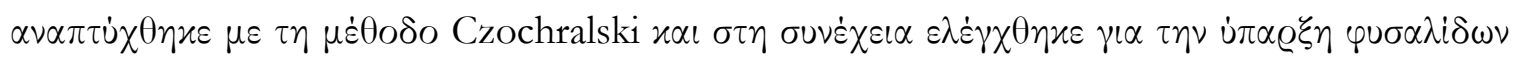

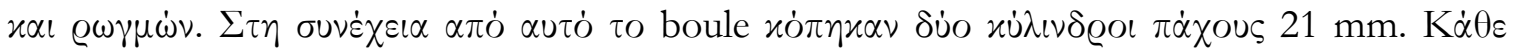

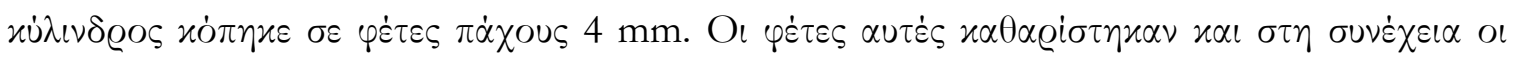

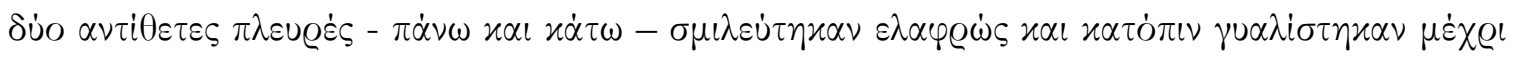

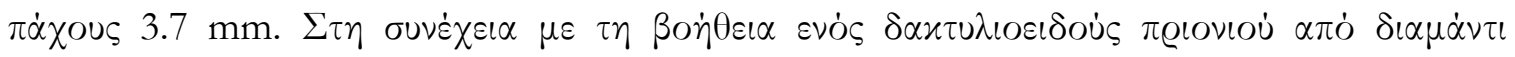

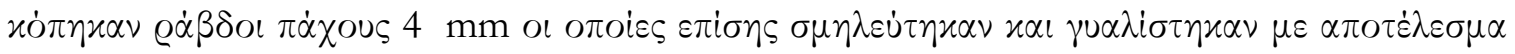

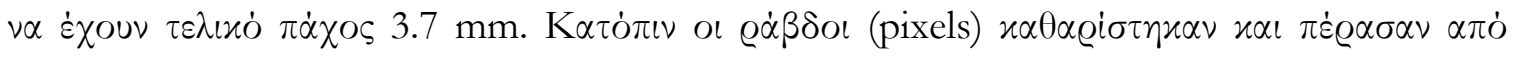

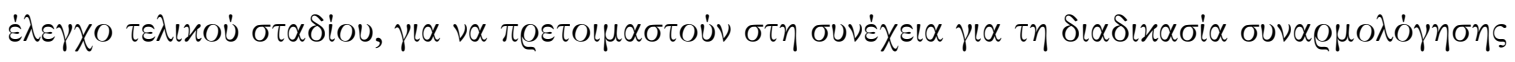

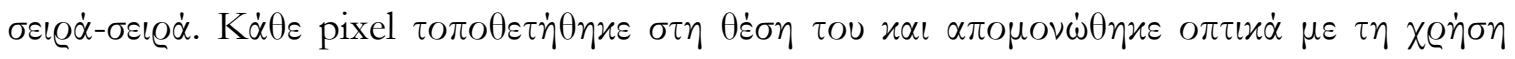

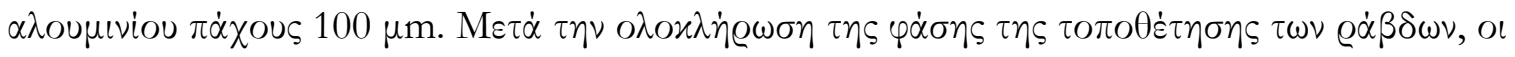

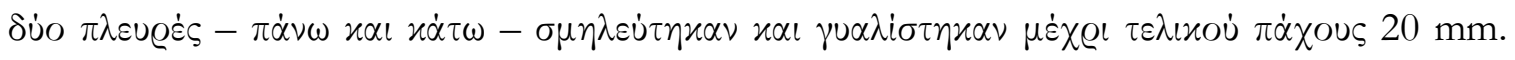

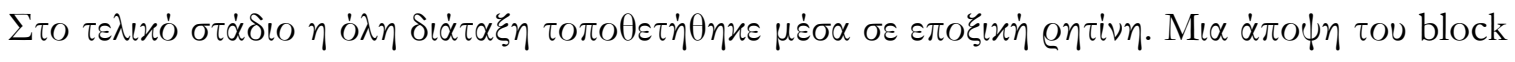
$\alpha \pi \dot{B}$ BGO $\varphi \alpha i v \varepsilon \tau \alpha \iota \sigma \tau O \sigma \chi \dot{\eta} \mu \alpha 1$.

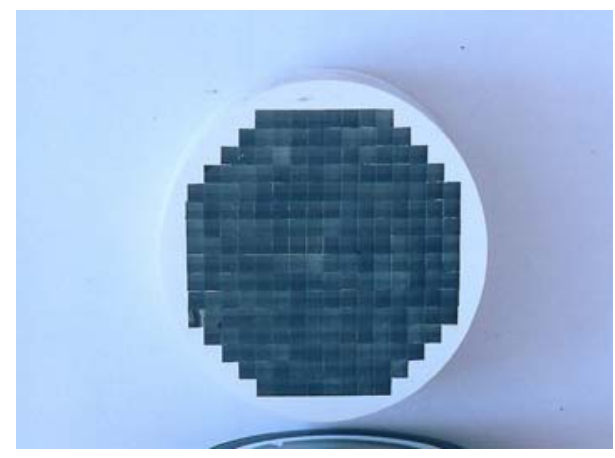

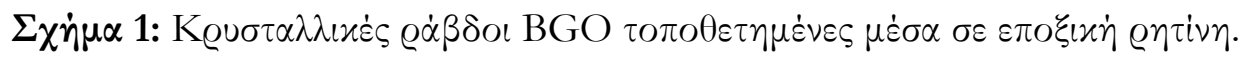




\subsection{2 $\Phi \omega \tau o \pi \circ \lambda \lambda \alpha \pi \lambda \alpha \sigma \iota \sigma \tau \dot{\eta} \varsigma$}

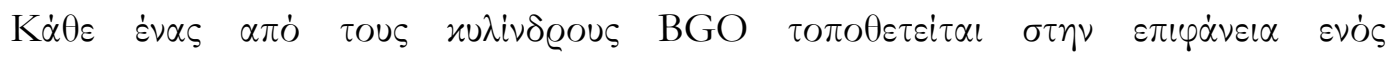

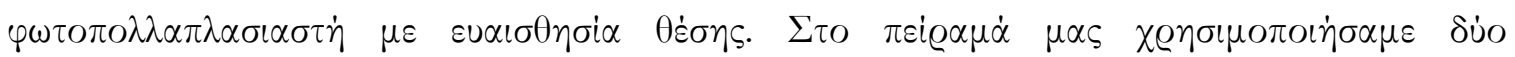

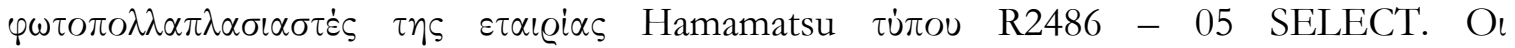

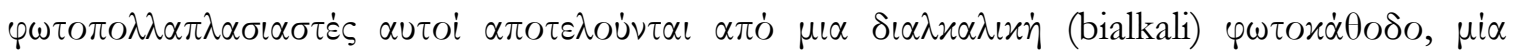

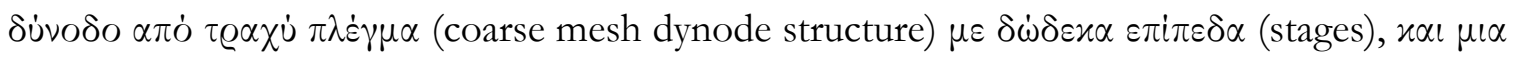

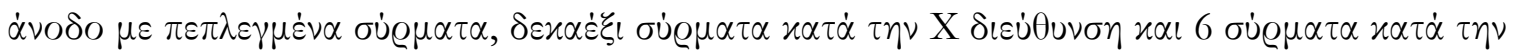

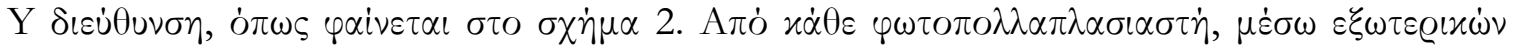

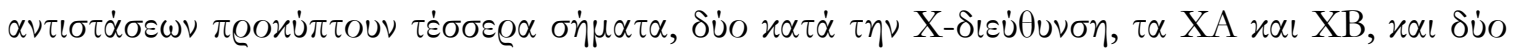

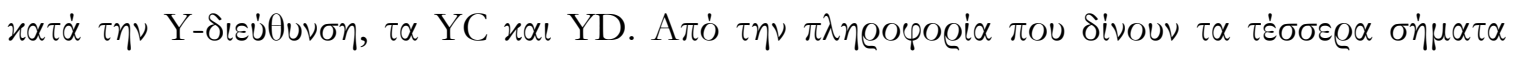

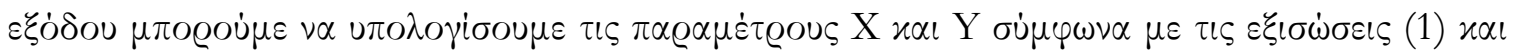
(2).

$$
\begin{aligned}
& X=\frac{X_{A}-X_{B}}{X_{A}+X_{B}} \\
& Y=\frac{Y_{C}-Y_{D}}{Y_{C}+Y_{D}}
\end{aligned}
$$

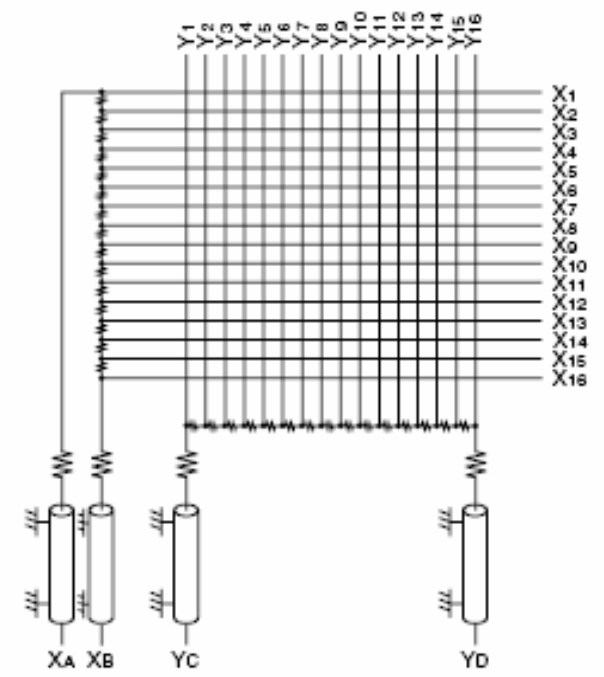

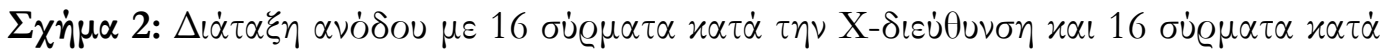

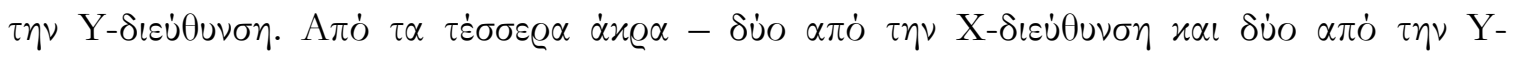




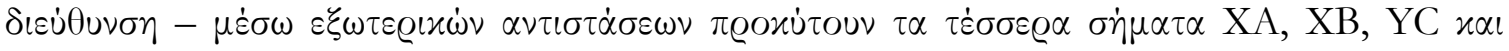
YD.

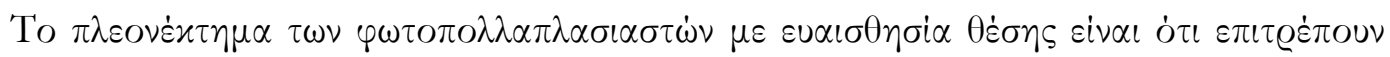

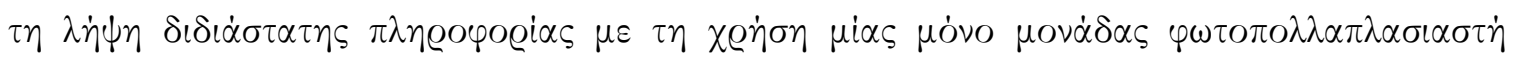

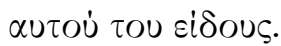

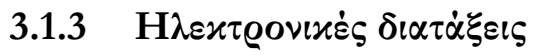

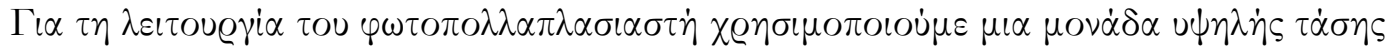

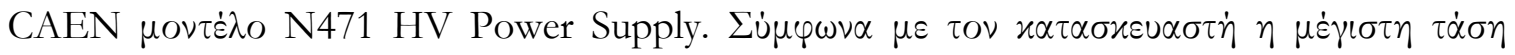

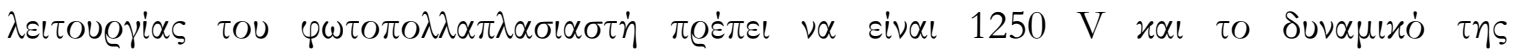

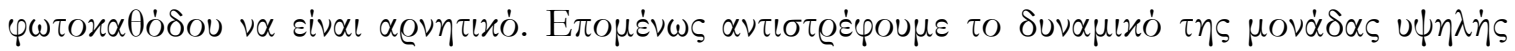

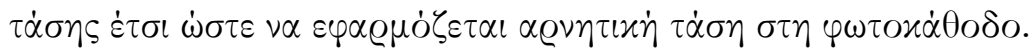

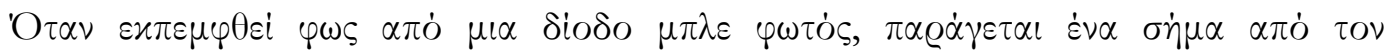

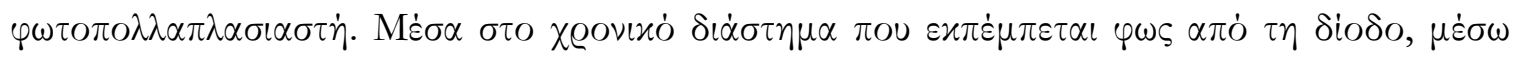

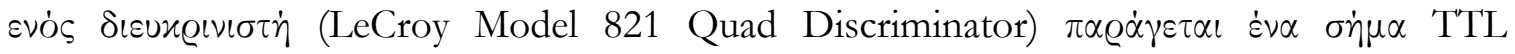

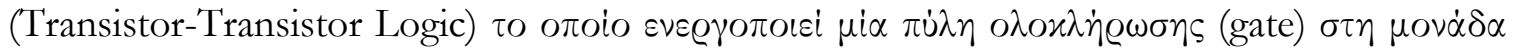

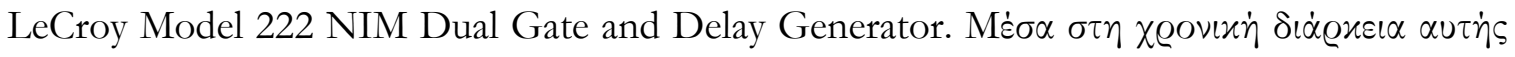

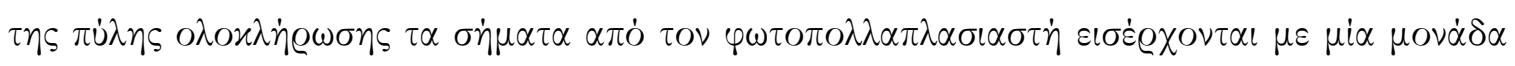

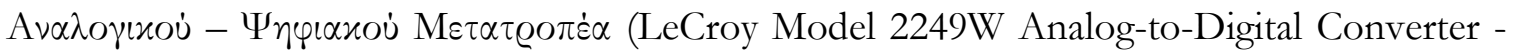

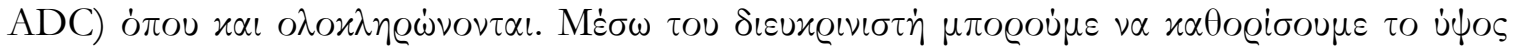

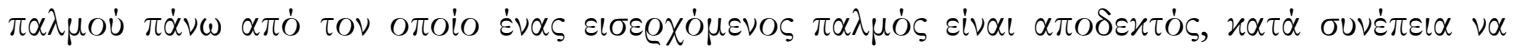

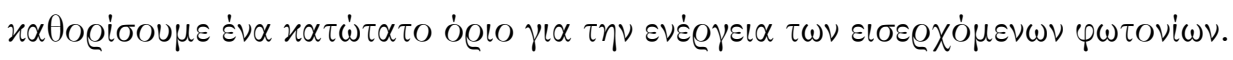

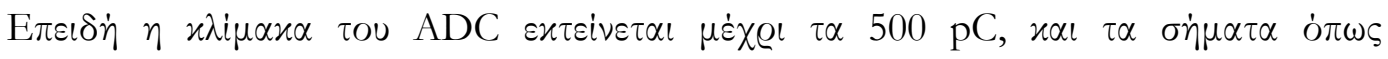

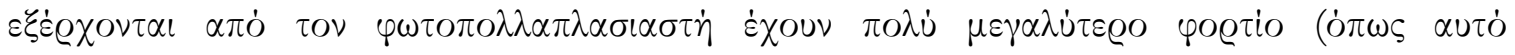

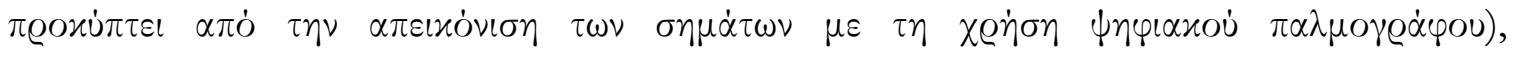

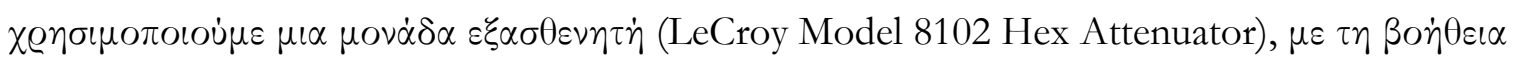

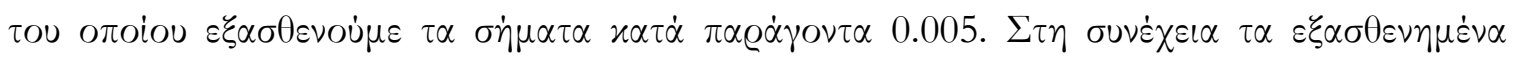

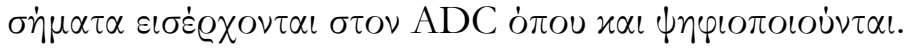

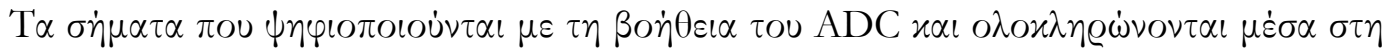

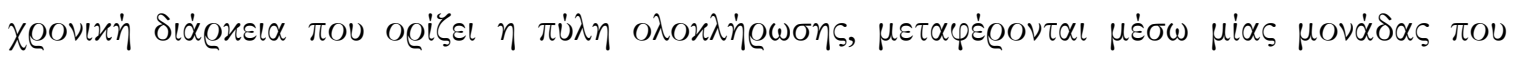




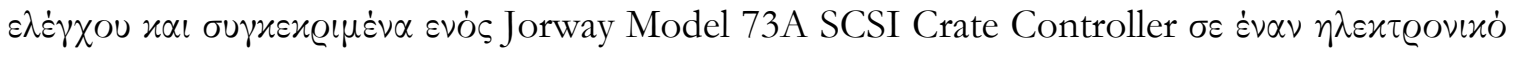

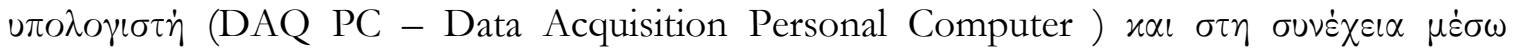

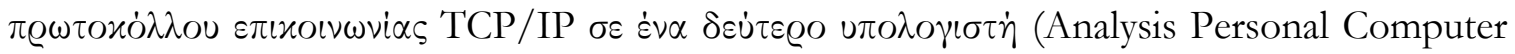

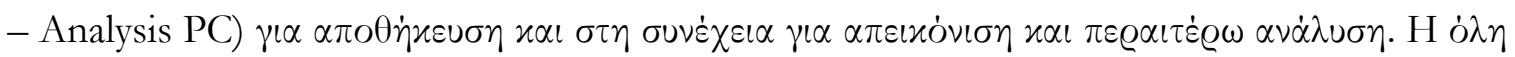

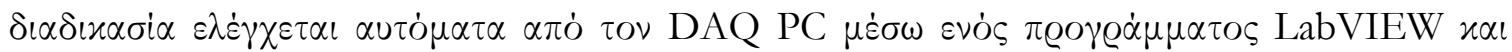

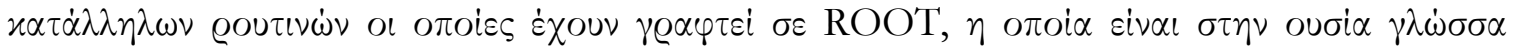

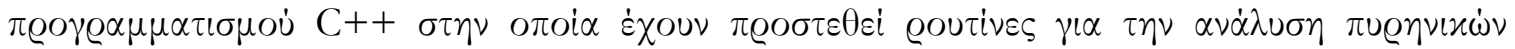
$\delta \varepsilon \delta о \mu \dot{\varepsilon} \nu \omega \nu$.

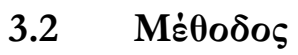

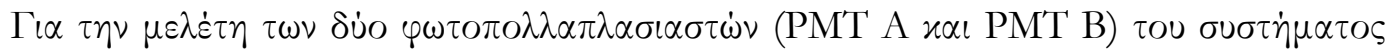
$\mu \alpha \varsigma, \chi \varrho \eta \sigma \mu \sigma_{0}$,

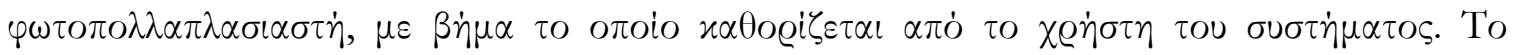

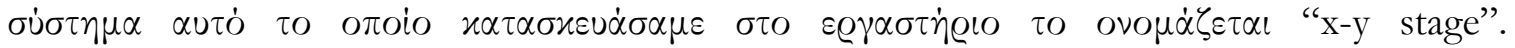

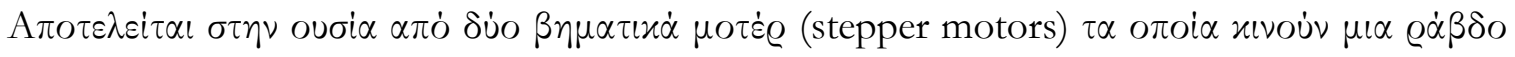

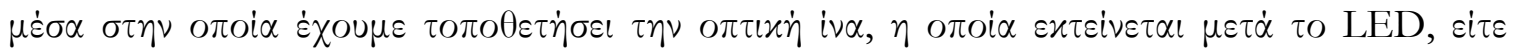

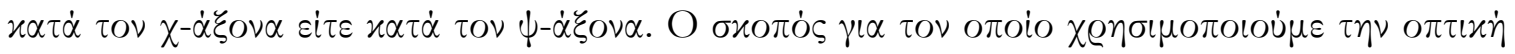

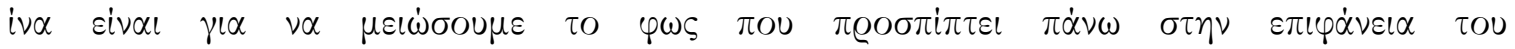

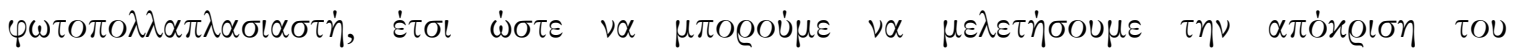

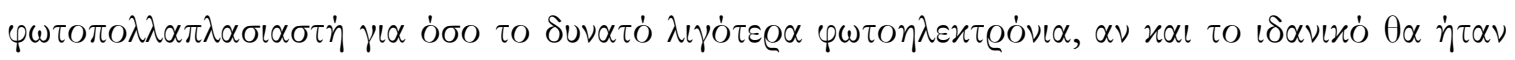

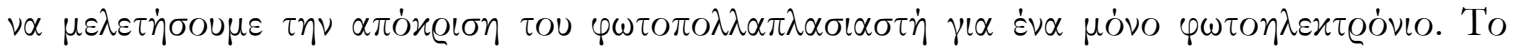

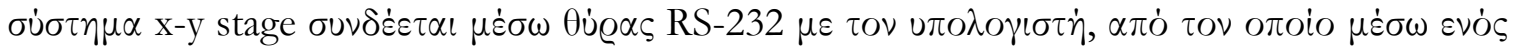

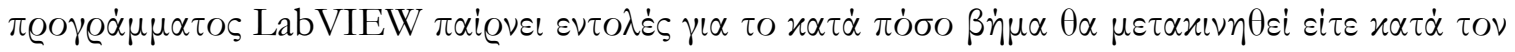
$\chi-\varepsilon i \tau \varepsilon \varkappa \alpha \tau \dot{\alpha} \tau O \nu \psi-\dot{\alpha} \xi o v \alpha$.

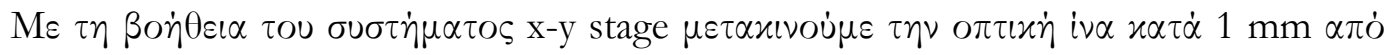

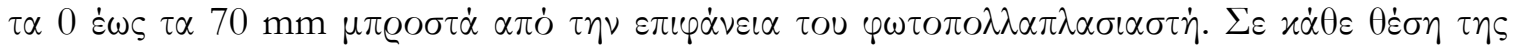

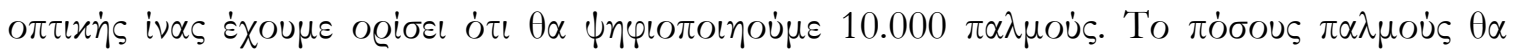

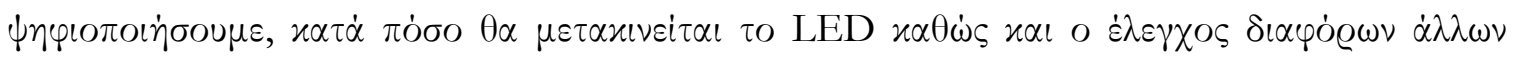

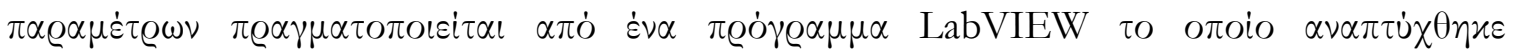

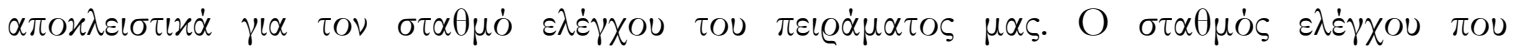

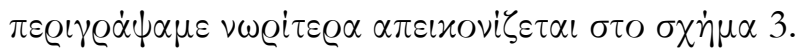




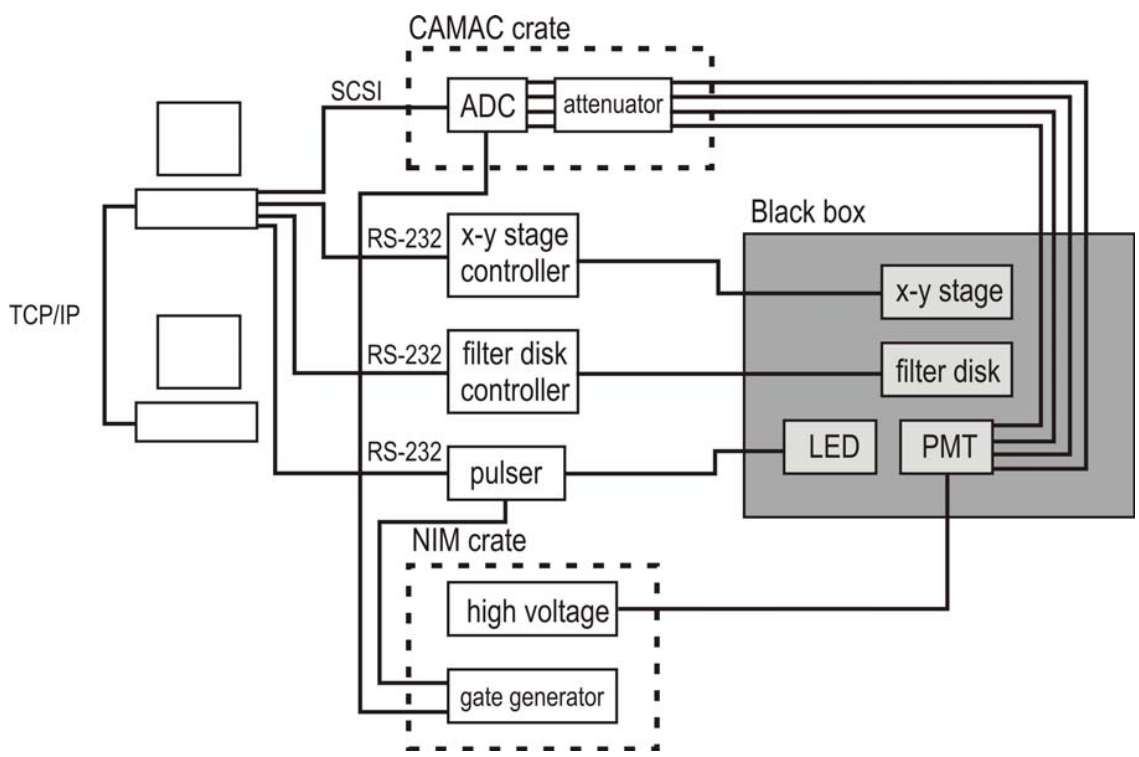

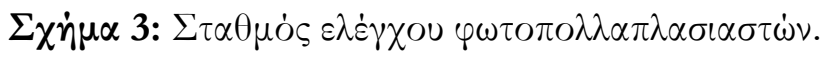

\section{A $\pi 0 \tau \varepsilon \lambda \dot{\varepsilon} \sigma \mu \alpha \tau \alpha$}

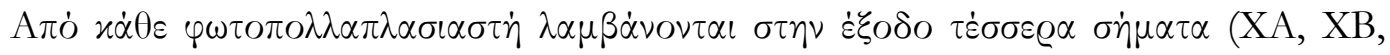

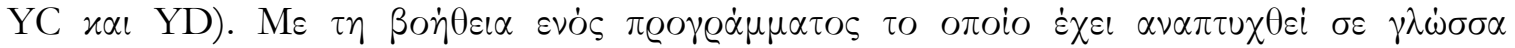

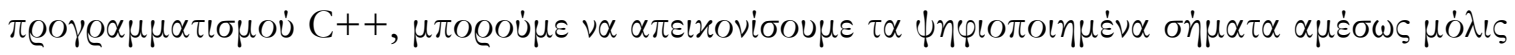

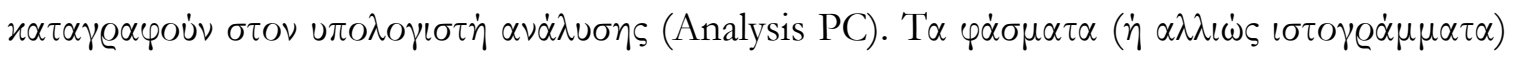

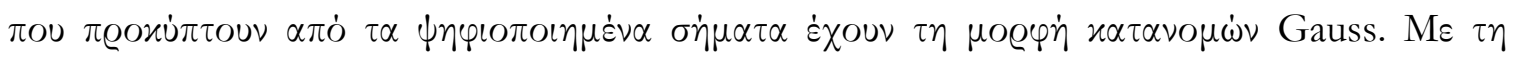

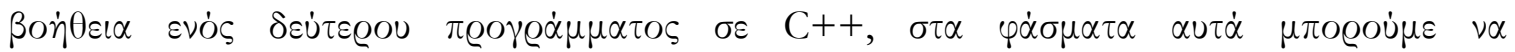

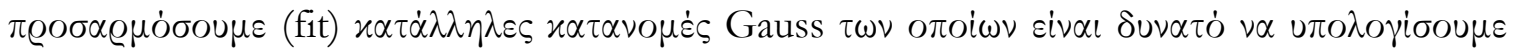

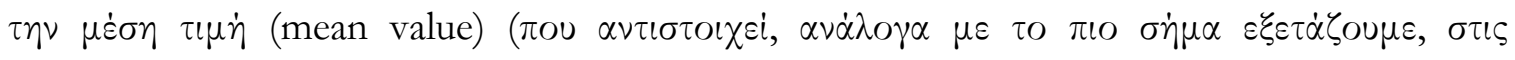
$\pi \alpha \varrho \alpha \mu \dot{\varepsilon} \tau \varrho O \nu \varsigma \mathrm{XA}, \mathrm{XB}, \mathrm{YC} \varkappa \alpha \iota \mathrm{YD}) x \alpha \iota \tau \eta \nu \tau u \pi \iota \dot{\eta} \alpha \pi \dot{\partial} x \lambda \iota \sigma \eta \sigma$.

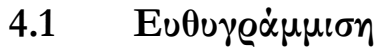

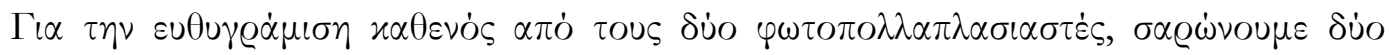

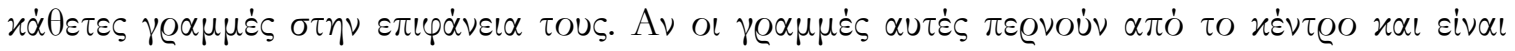

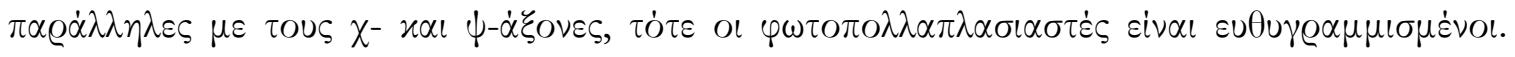

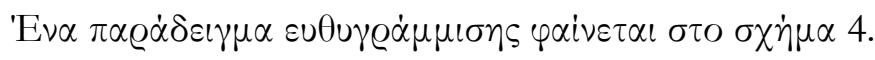




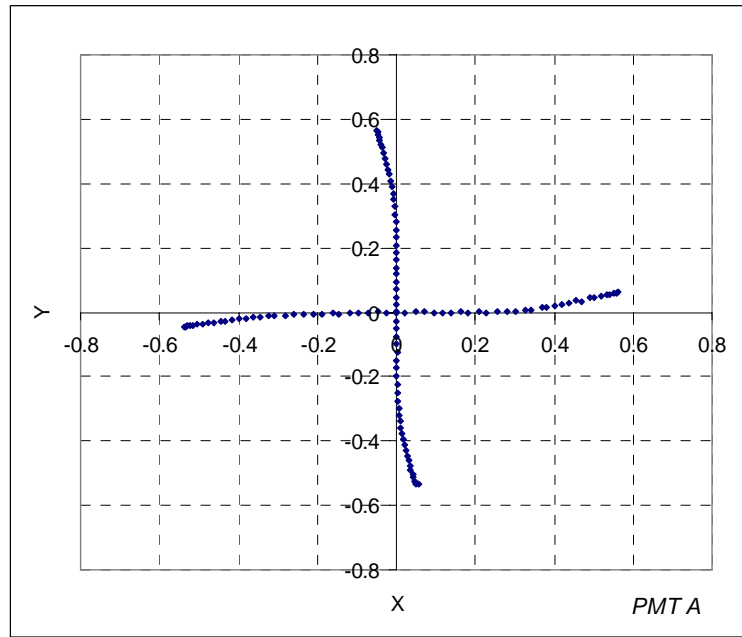

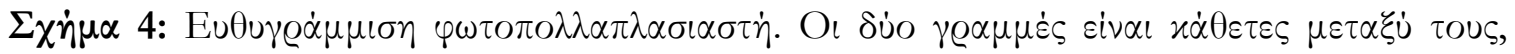

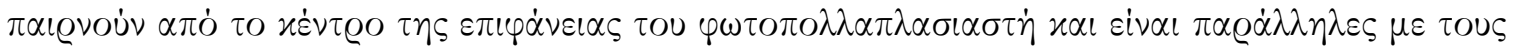
$\chi-x \alpha \iota \psi-\dot{\alpha} \xi o v \varepsilon \varsigma$.

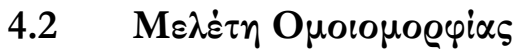

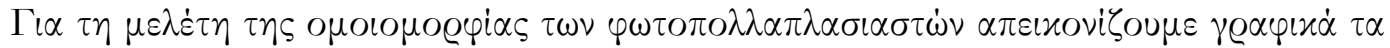

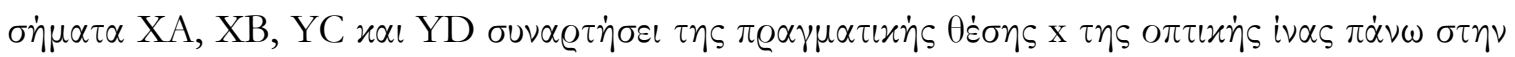

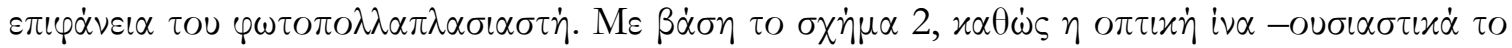

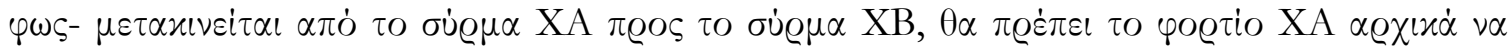

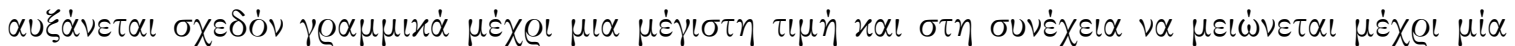

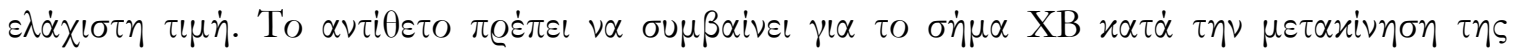

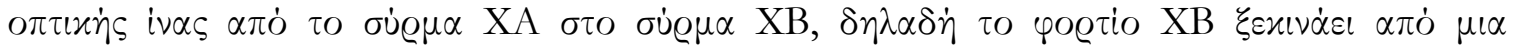

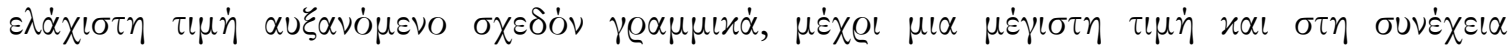

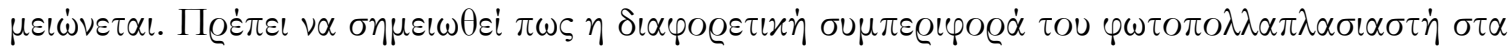

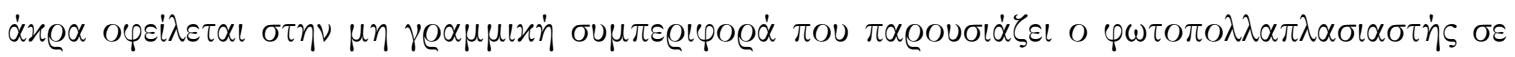

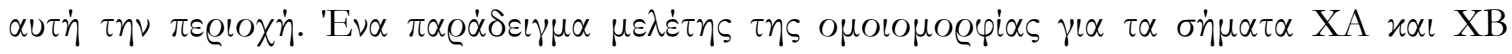

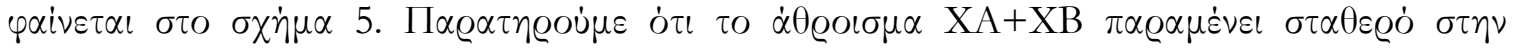

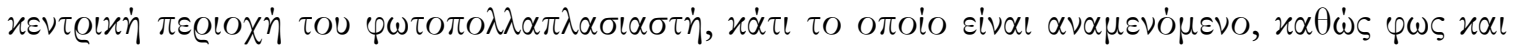

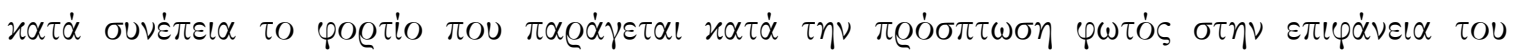

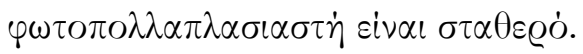




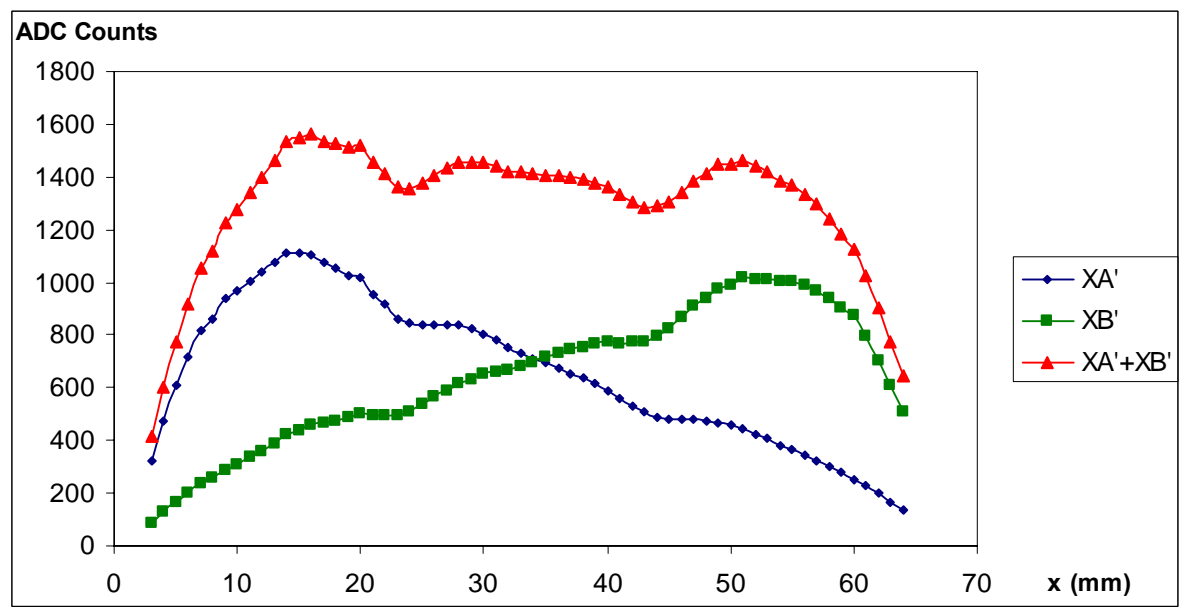

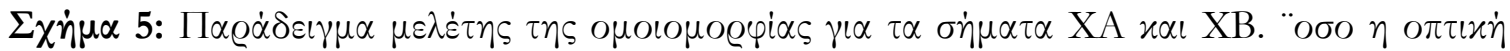

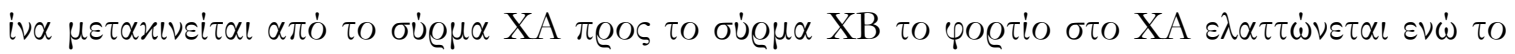

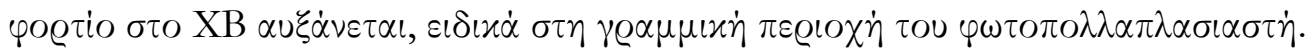

\section{3 $\Pi \lambda \dot{\eta} \varrho \eta s \Sigma \dot{\alpha} \varrho \omega \sigma \eta$}

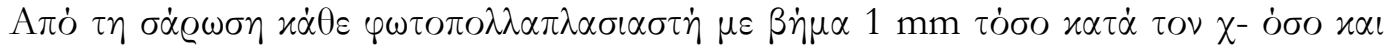

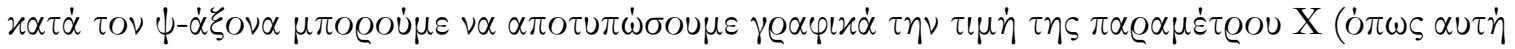

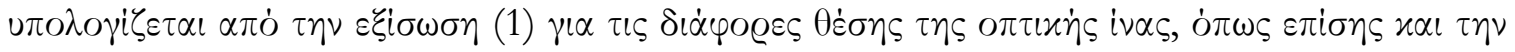

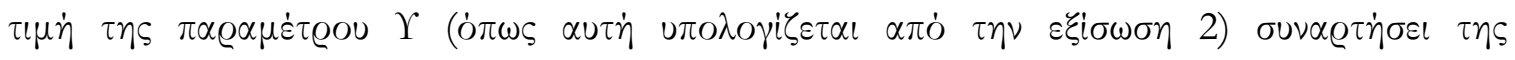

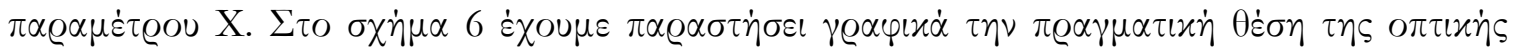

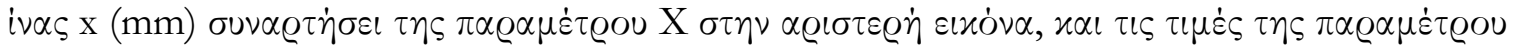

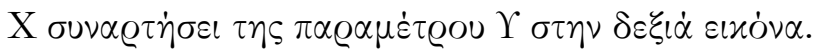
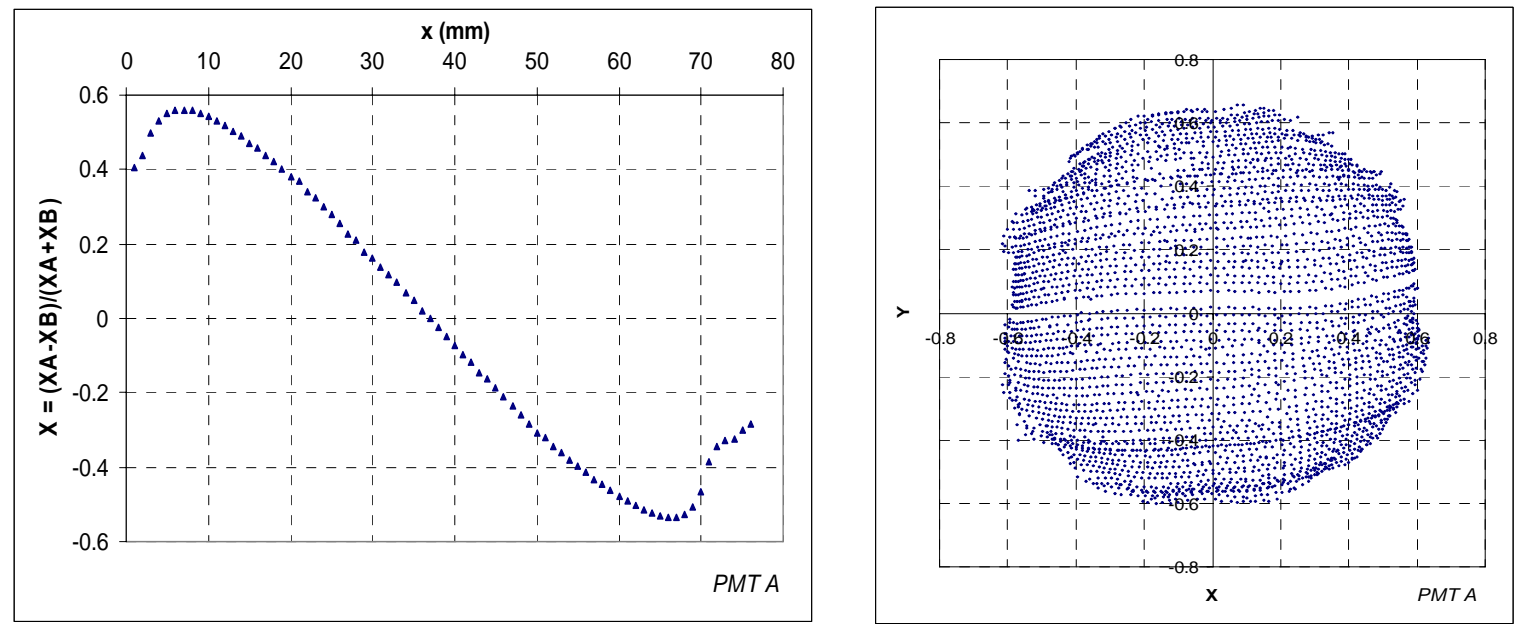


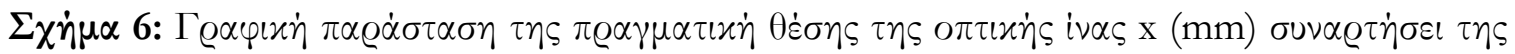

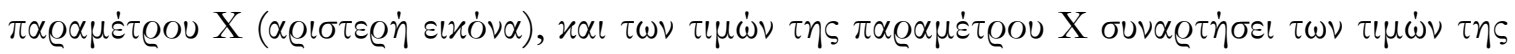

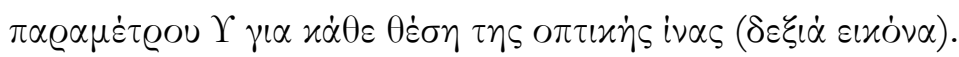

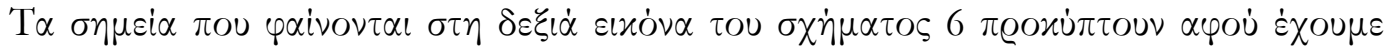

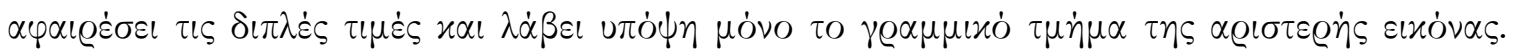

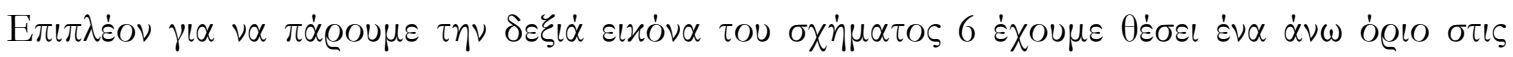

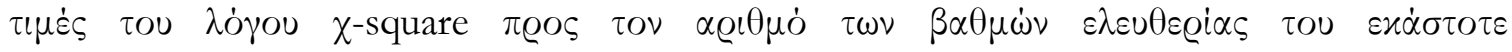

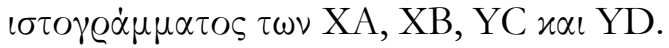

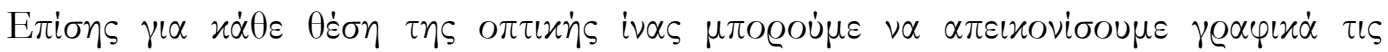

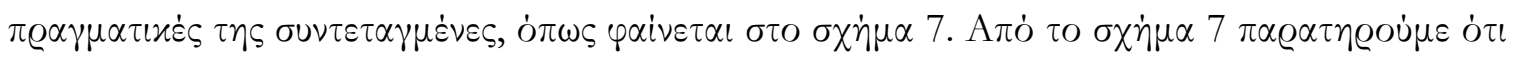

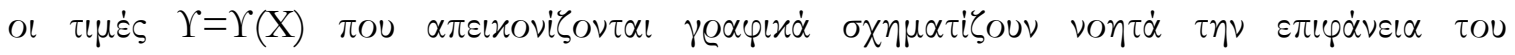

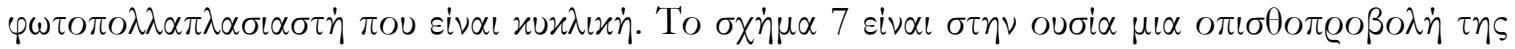

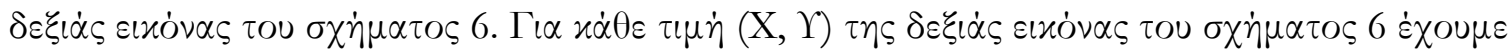

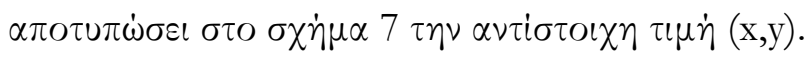

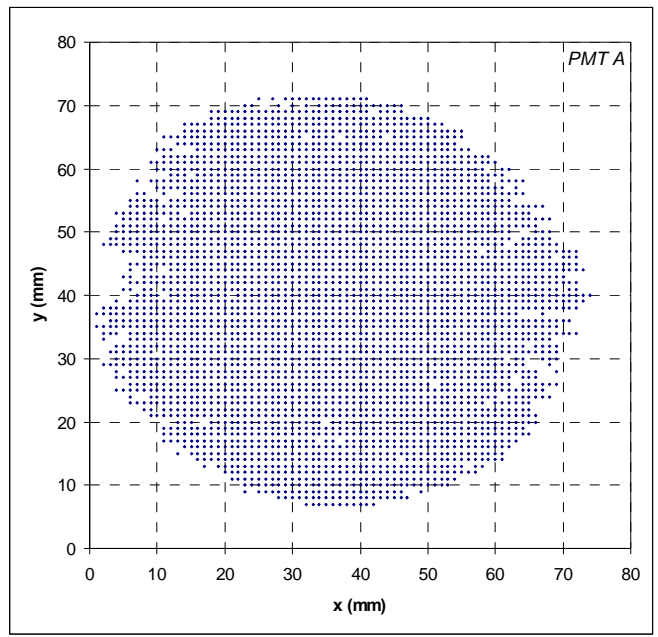

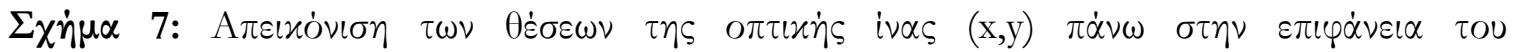
$\varphi \omega \tau O \pi \circ \lambda \lambda \alpha \pi \lambda \alpha \sigma \iota \alpha \sigma \tau \dot{\eta}$.

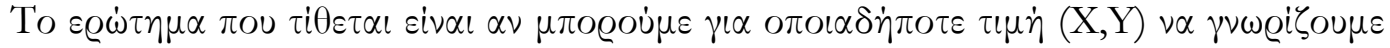

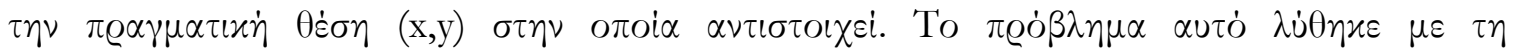

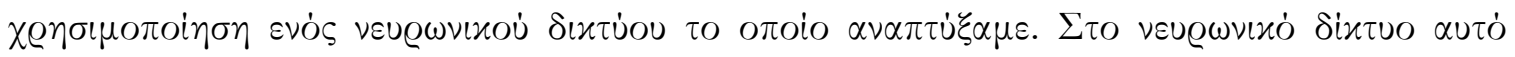




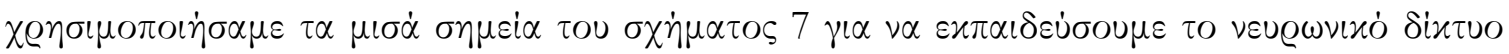

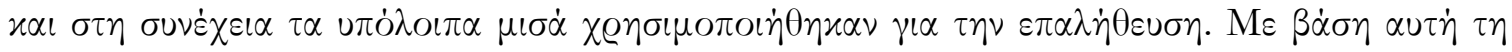

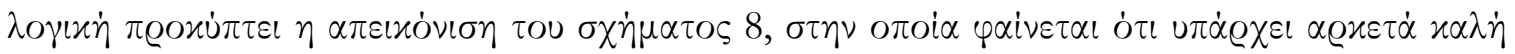

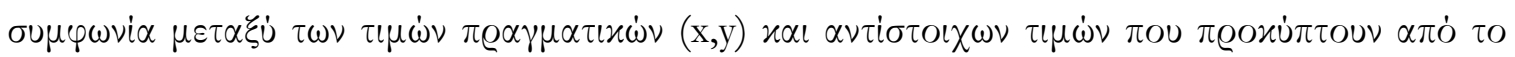

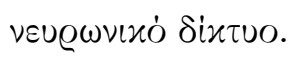

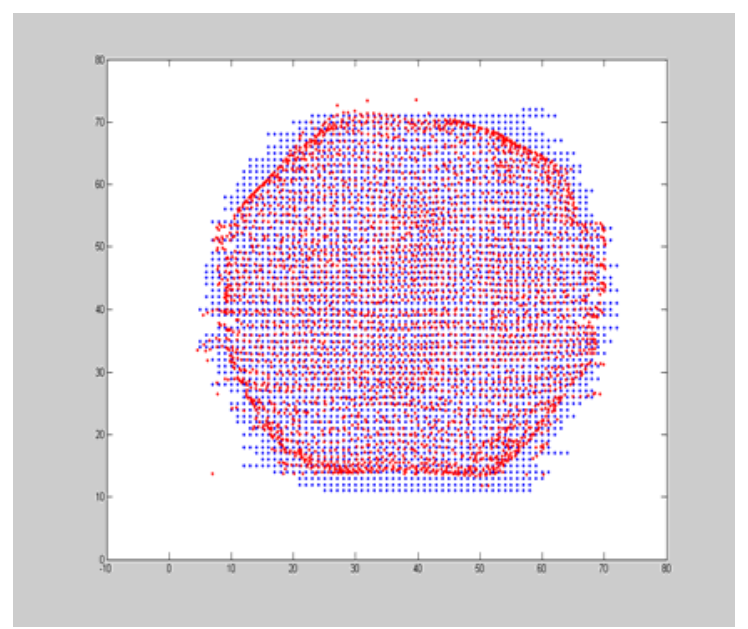

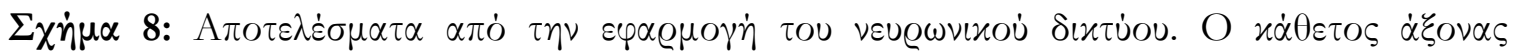

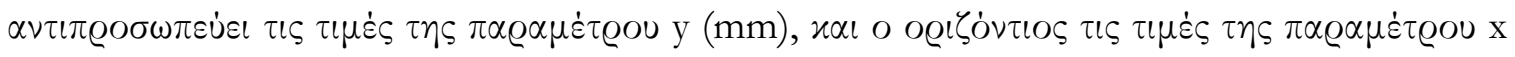

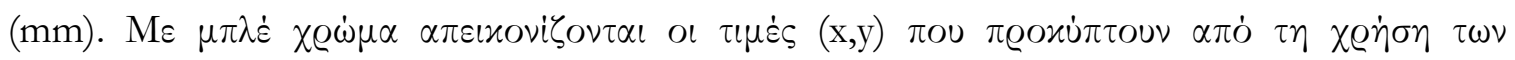

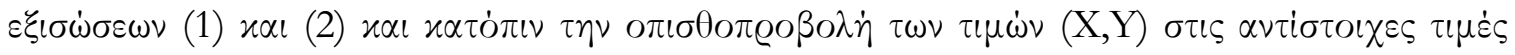

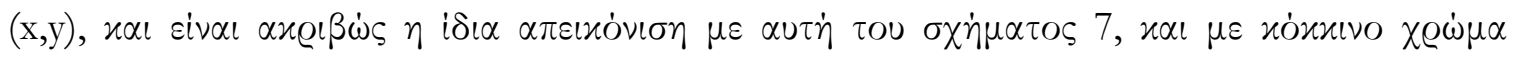

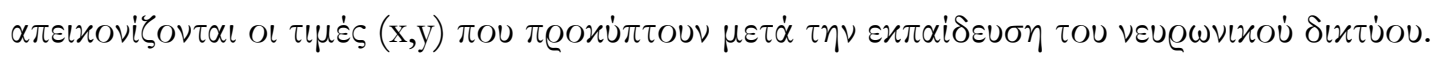

\section{5. $\Sigma \cup \mu \pi \varepsilon \varrho \dot{\alpha} \sigma \mu \alpha \tau \alpha$}

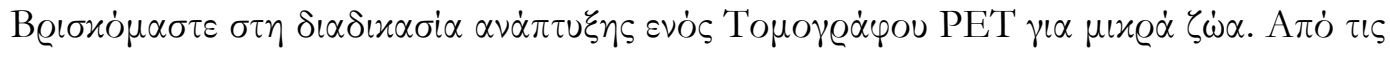

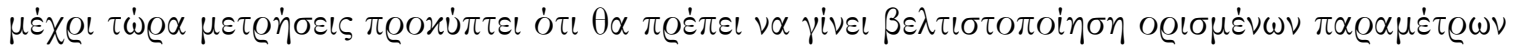

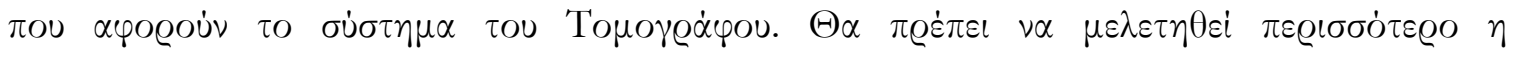

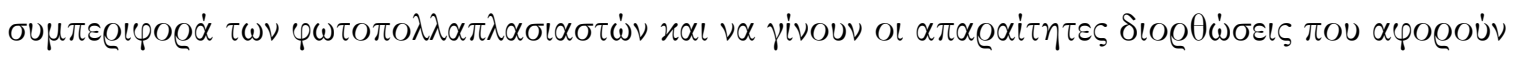

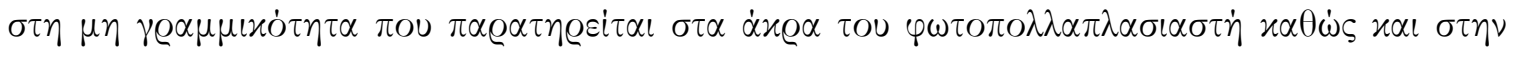

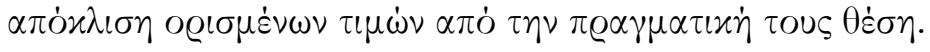

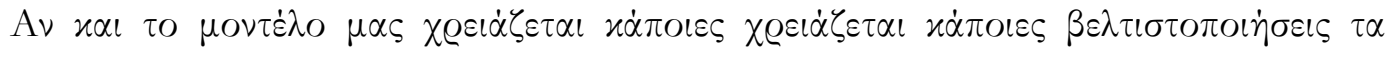

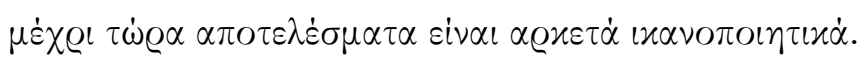




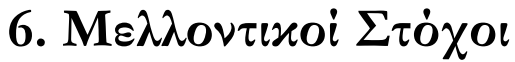

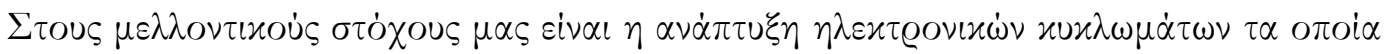

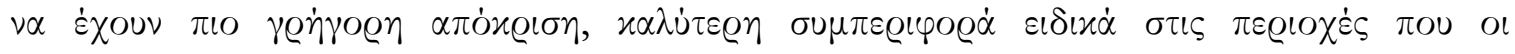

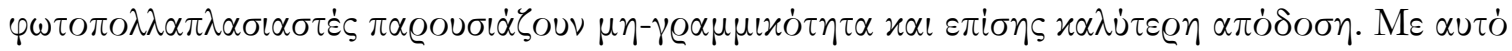

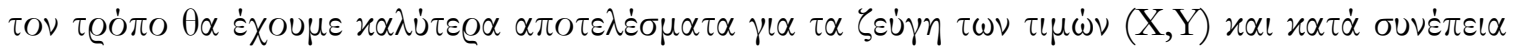

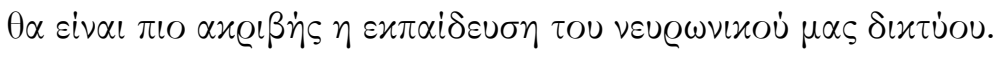

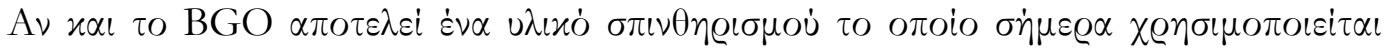

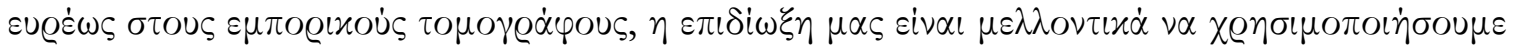

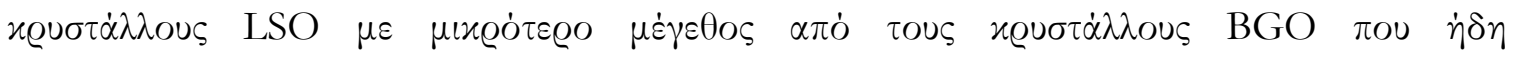

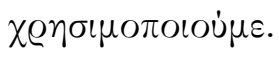

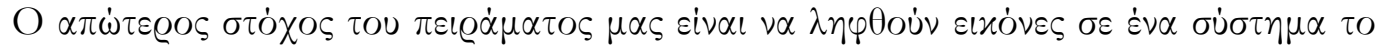

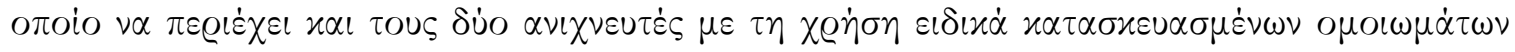

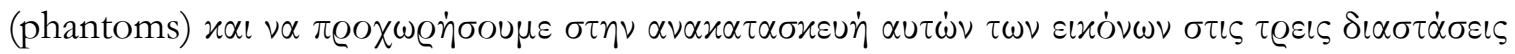

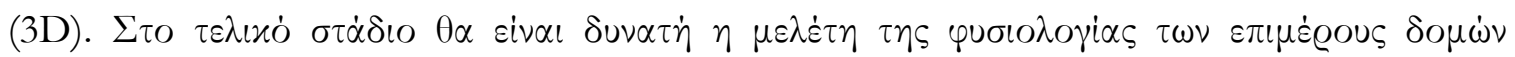

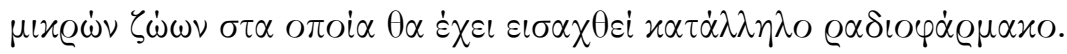




\section{Project Overview}

\section{Introduction}

Positron Emission Tomography, often referred to by its acronym, PET, is an emerging radiologic modality that yields transverse tomographic images of functioning organs in the human body. Although the positron has already been first theoretically described by Paul Dirac and then discovered in 1932 by C. Anderson, it was not until early 1950s that the medical imaging possibilities of a particular class of positron-emitting radioisotopes were first discovered. PET enables the metabolic imaging of organs (as opposed to the anatomic imaging provided by techniques such as the X-ray imaging or the Computerized Tomography (CT)), in molecular level, and this is the reason why it is characterized as molecular imaging.

Especially, small animal PET tomographs, require high spatial resolution and can play an important role in biology [Myers 2001, Herschman 2003] and studies of in vivo tracer pharmacokinetics and metabolism [Wang 2005, Sossi 2005, Toyama 2005, Kenanova 2005]. A first realization of a small animal PET scanner by Watanabe et al. was published in Ref. [Watanabe 1992]. In the ensuing years several designs of small animal PET systems 
were realized. Various implementations have been reported in the literature using a variety of detector and design technologies.

\section{The Problem}

We have been developing a small animal PET prototype, in order to study specific design characteristics and measure its performance. The basic block detector design consists of a $16 \times 16$ array of $3.75 \times 3.75 \times 20 \mathrm{~mm}^{3}$ individual BGO crystals coupled to a Hamamatsu R2486 PS-PMT. Since this PMT is cylindrical, the $16 \times 16$ array is being built omitting the corner crystals, becoming thus an array of 216 crystals in a cylindrical configuration. Measurements of the individual detector performance as well as measurements of the PSPMTs have been performed.

In order to study the individual components (photomultiplier tube and then the whole block detector) we have developed a test stand and the appropriate software to perform the required tests automatically.

The first problem that was posed was the study of the response of the two photomultiplier tubes of the system when light was incident on their surface. In order to solve that problem we have developed a test stand where the photomultiplier tubes were mounted to the appropriate electronic circuits. The whole process of study is automatically controlled by a set of computers and their response is registered in digital form.

The next problem that was posed was how to use the digitized data to interpret the response of the photomultiplier tubes. We had to display the data in an appropriate form and then develop the appropriate software to do the analysis of these data.

Another problem that was posed was what protocol to apply for the testing of the photomultiplier tubes. The need to test the photomultiplier tubes as extensively as possible led to the development of a system that moves the light source in front of the photomultiplier tube step-wise. 


\section{Thesis Originality}

The problem of the development of a test station that would allow us to test the individual components of the Small Animal PET led to the development of a test stand.

In order to test the photomultiplier tubes we have developed a mechanical system to immobilize each photomultiplier tube while being tested and we have also developed the $x-y$ stage which is the system used to move the light source (in our case the optical fiber which is connected to the LED). The software used to move the stepper motors of the $x-y$ stage in preset steps is controlled by a program we have developed in LabVIEW. Moreover the program that automatically controls the data acquisition process was developed also using LabVIEW. The data at the final stage of the data acquisition process are displayed with a program that was developed using $\mathrm{C}++$ with ROOT routines. $\mathrm{C}++$ was also used to do the fitting of the digitized signals with Gauss distributions.

One quite innovative feature in our project is the use of artificial neural networks to determine the real position of the optical fiber $(\mathrm{x}, \mathrm{y})$ when applying as input the values of the parameters $(\mathrm{X}, \mathrm{Y})$, as explained in Chapter 5.

\section{Publications}

\section{Articles in journals:}

G. Tzanakos, M. Nikolaou, D. Drakoulakos, D. Karamitros, G. Kontaxakis, E. Logaras, G. Panayotakis, S. Pavlopoulos, M. Skiadas, G. Spyrou, T. Thireou, and D. Vamvakas, "Design Considerations and Construction of a Small Animal PET Prototype”, In Press, Corrected Proof, Nuclear Instruments and Methods in Physics Research Section A.

\section{Published contributions to academic conferences:}

1. G. Tzanakos, N. Apostolou, X. Benetou, G. Karmaniolas, G. Kontaxakis, M. Nikolaou, G. Panayotakis, S. Pavlopoulos, M. Skiadas, G. Spyrou, T. Thireou, "Development of a Small Animal PET Prototype", 2000 IEEE Medical Imaging Conference, Conference Record, 3: 21_53-21_56, October 2000. 
2. M.E. Nikolaou, G. Spyrou, G. Panayiotakis, G. Tzanakos, "Design studies of collimated gamma ray sources”, 2000 IEEE Medical Imaging Conference, Conference Record, 3: 20_100 - 20_104, October 2000.

3. G. Tzanakos, D. Georgakakis, G. Kontaxakis, M. Skiadas, N Apostolou, S. Pavlopoulos, T. Thireou, M. Nikolaou, G. Panayiotakis and G. Spyrou, "Design of a PET tomograph for small animal research", 2001 IEEE Medical Imaging Conference, Conference Record (in CD-ROM, M9A-2), October 2001.

4. M. Skiadas, G. Kontaxakis, M. Nikolaou, G. Panayotakis, S. Pavlopoulos, G. Spyrou and T. Thireou, "Design of a prototype PET tomograph", 8th Biannual PanHellenic Nuclear Medicine Congress, Conference Record, p. 53, April 2002.

5. G. Tzanakos, G. Kontaxakis, M. Nikolaou, G. Panayotakis, S. Pavlopoulos, M. Skiadas, G. Spyrou and T. Thireou, "Design and Construction of a Small Animal PET Prototype," Conference Record of the 2002 IEEE Nuclear Science and Medical Imaging Conference (in CD-ROM), Norfolk, Virginia, USA, November 2002.

6. S. Pavlopoulos, D. Drakoulakos, D. Karamitros, G. Kontaxakis, E. Logaras, M. Nikolaou, G. Panayotakis, M. Skiadas, G. Spyrou , T. Thireou, G. Tzanakos and D. Vamvakas, "Design Considerations and Initial Evaluation Results of a Small Animal PET Prototype", Book of Abstacts, p. 164 (M5-122), 2004 IEEE Medical Imaging Conference, Rome, Italy, October 2004.

7. M. Nikolaou, D. Drakoulakos, D. Karamitros, G. Kontaxakis, E. Logaras, G. Panayotakis, S. Pavlopoulos, M. Skiadas, G. Spyrou, T. Thireou, G. Tzanakos and D. Vamvakas, "Tests and Measurements for Characteristics Optimization of Photomultiplier Tubes used in a Positron Emission Tomography System" Book of Abstacts, p. 217 (M10-148), 2004 IEEE Medical Imaging Conference, Rome, Italy, October 2004.

8. D. Drakoulakos, D. Karamitros, G. Kontaxakis, E. Logaras, M. Nikolaou, G. Panayotakis, S. Pavlopoulos, M. Skiadas, G. Spyrou, T. Thireou, D. Vamvakas and G. Tzanakos, "Design Considerations and Construction of a Small Animal PET Prototype," 3rd International Conference on Imaging Technologies in Biomedical Sciences, Milos, Greece, September 2005. 


\section{Financial Support}

This program was partially funded by the European Social Fund (ESF), Operational Program for Educational and Vocational Training II (EPEAEK II), and particularly by the Program HERAKLEITOS.
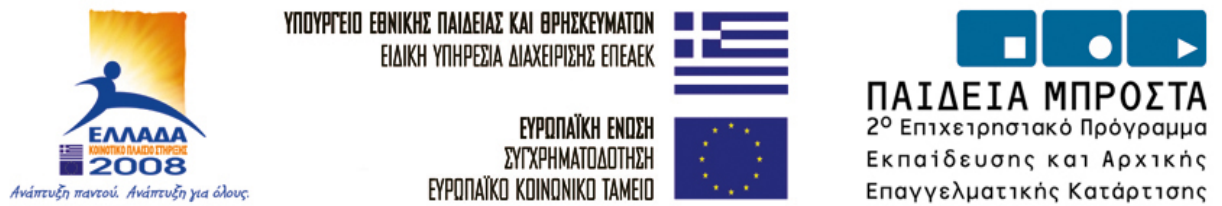


\section{Introduction}

Positron Emission Tomography, often referred to by its acronym, PET, is an emerging radiologic modality that yields transverse tomographic images of functioning organs in the human body. Although the positron has already been first theoretically described by Paul Dirac and then discovered in 1932 by C. Anderson, it was not until early 1950s that the medical imaging possibilities of a particular class of positron-emitting radioisotopes were first discovered. PET enables the metabolic imaging of organs (as opposed to the anatomic imaging provided by techniques such as the X-ray imaging or the Computerized Tomography (CT)), in molecular level, and this is the reason why it is characterized as molecular imaging.

Especially, small animal PET tomographs, require high spatial resolution and can play an important role in biology [Myers 2001, Herschman 2003] and studies of in vivo tracer pharmacokinetics and metabolism [Wang 2005, Sossi 2005, Toyama 2005, Kenanoca 2005]. A first realization of a small animal PET scanner by Watanabe et al. was published in Ref. [Watanabe 1992]. In the ensuing years several designs of small animal PET systems 
were realized. Various implementations have been reported in the literature using a variety of detector and design technologies [Lecomte 1996, Bloomfield 1997, Bruyndoncky 1997, Cherry 1997, Fries 2997, Del Guerra 1998, Chatziioannou 1999, Huber 1999, Jeavons 1999 , Siegel 1999, Weber 1999, Myers 2002, Chatziioannou 2002, Stickel 2005, Tai 2005].

The basic technology for these scanners is based on small inorganic crystals, mainly from BGO, GSO, and LSO, forming detector blocks read out by position sensitive and multianode PMTs. BGO is the material used in a lot of commercial scanners, having replaced NaI, mainly because BGO has higher stopping power and it is not hygroscopic. LSO has more or less the same stopping power but it is much faster, with a higher photon yield. These properties make it appropriate for smaller coincidence window and faster scanners. On the negative side, LSO is much more expensive than BGO and has some inherent radioactivity.

We have been developing a small animal PET prototype, in order to study specific design characteristics and measure its performance. The basic block detector design consists of a $16 \times 16$ array of $3.75 \times 3.75 \times 20 \mathrm{~mm}^{3}$ individual BGO crystals coupled to a Hamamatsu R2486 PSPMT. Since this PMT is cylindrical, the $16 \times 16$ array is being built omitting the corner crystals, becoming thus an array of 216 crystals in a cylindrical configuration. We should note that in our initial design [Pavlopoulos 1992, Pavlopoulos 1996, Tzanakos 1993] we used the square R2487 PSPMT, the production of which is now stopped. Measurements of the individual detector performance as well as measurements of the PSPMTs have been performed. The two detector blocks which will form the basic tomographic unit have been assembled. We note that using non-square blocks will introduce some difficulties in the image reconstruction which are currently under study. 


\section{Chapter 1}

\section{Principles of Positron Emission Tomography}

\subsection{History of PET}

The potential of positron imaging and the value of eliminating the collimator was recognized by the early developers of nuclear medicine instrumentation, long before the launch of reconstruction algorithms which could allow the generation of transverse sections from data covering a large number of angles. G. Brownell and his group developed a family of instruments over several decades that demonstrate the evolution of PET first from dual planar detectors [Brownell et al 1969] working in coincidence and providing longitudinal tomographic images, progressing from there to rotating detectors and using transverse reconstruction algorithms to obtain transverse sections and finally to complete circular stationary detector arrangements, first with a single slice (Burnham et al 1983) and subsequently using an increased number of transverse slices (Burnham et al 1985). Hal Anger also worked on positron imaging almost as soon as he developed the single photon Anger camera [Anger 1966]. His dual detector PET scanner was made commercially 
available for a brief period by Nuclear Chicago Corporation, but early clinical data quickly pointed out the main problem with PET imaging: while the elimination of the collimator increased the photon flux hitting the detectors by more than an order of magnitude relative to single photon imaging, the fraction of event which are found in coincidence is of the order of $1 \%$, therefore requiring a high singles count rate capability to achieve an acceptable coincidence rate.

It is also interesting to note that time-of-flight (TOF) imaging was a gleam in the eyes of these early investigators [Brownell 1969], leading Brownell to conclude in 1969 that with that time state-of-the-art scintillators and photomultiplier tubes, TOF-which increases in benefit as the object size increases-was useful for large objects, but not yet for humans. TOF then went through a period of revival in the 1980s and is only now being revived for a third time, as will be described in more detail below.

The instrumentation efforts before about 1975 suffered not only from a lack of knowledge about transverse section reconstruction methods, but also from a severe lack of computing power. Longitudinal tomographic images were often obtained with dedicated electronic processing methods instead of superposition of data using digital techniques, since even a single image with a $64 \times 64$ matrix was considered to require a large amount of storage space. At the time the primary potential clinical application was bone scanning with F-18 and some of the early positron bone scans were of stunning quality by the standards of that time, demonstrating the ability of PET to combine excellent spatial resolution with high sensitivity.

Early efforts to achieve transverse section images in nuclear medicine relied on iterative methods [Muehllehner 1971, Kuhl 1973], but this changed rapidly with the introduction of x-ray computed tomography and the filtered backprojection technique. This allowed a series of PET scanners to be developed at a number of academic institutions [TerPogossian 1978, Phelps 1976, Burnham 1983, Bohm 1978, Senda 1985, Cho 1983, Derenzo 1981, Wong 1984], using first $\mathrm{NaI}(\mathrm{Tl})$ in a hexagonal arrangement [Phelps 1976], a 1-to-1 crystal-to-photomultiplier tube (PMT) coupling and involving transverse scanning and wobbling motions, finally leading to the use of BGO in a circular arrangement operating in stationary mode [Thompson 1979, Cho 1977]. It also changed the approach of the efforts made, starting with multiple longitudinal planar images to the reconstruction of transverse section using dual rotating detectors, culminating in the development of a fully 3D 
acquisition [Muehllehner 1976] and reconstruction [Colsher 1980] in the late 1970s. After the introduction of transverse section reconstruction using filtered backprojection, PET imaging entered a period of rapid advancement and increased its use in a number of leading research institutions, particularly because this also coincided with the development of FDG [Reivich 1979] which created an opportunity to study brain function in a way that was impossible up to that point.

Today, there are over 400 PET scanners worldwide.

\subsection{PET unit}

A positron emission tomography unit contains the following equipment:

A cyclotron, where the appropriate radionuclides are produced while bombarding nuclei with protons and other particles, resulting in $(\mathrm{p}, \mathrm{n})$ or $(\mathrm{p}, \mathrm{a})$ type interactions with the synchronous production of radionuclides which emit the positrons used in PET. Several types of cyclotrons exist, one of the most convenient is the baby cyclotron which has the advantage that it operates close to the PET system for the immediate use of the radionuclides obtained in clinical applications.

A radiochemical laboratory, for the synthesis and quality control of radiopharmaceuticals.

A PET scanner for data acquisition, and powerful computers to register and process the acquired data and perform the image reconstruction.

\subsection{Cyclotron}

A cyclotron consists of two D-shaped electrodes known as dees. The dees exist between the poles of a powerful electromagnet. The cyclotron is an accelerator of subatomic particles. It produces a large quantity of protons (heavy particles with an electrical positive charge) and gets them moving at an accelerated rate along a circular orbit, inside a chamber controlled by powerful alternating electromagnetic fields. Thus, the particles gain energy and are ejected against a target at nearly the speed of light. The atoms of a substance placed in this target are transformed by this bombardment into radioactive, unstable isotopes, by means of a nuclear reaction. 
There are many radioactive isotopes that can be produced in the cyclotron. In order to use them in a PET apparatus for imaging the body, they must:

be capable of emitting positrons when they decay radioactively, i.e., when they transform from an unstable isotope into another, stable one,

have a short period of instability, i.e., it will take a few minutes for any given atom of this isotope to emit a positron and become a stable element. The average measure of this instability is called half-life, which is the time it takes to decrease by half the number of radioactive isotopes of that element,

be readily incorporated into an useful radiopharmaceutical, by chemical synthesis. Since carbon, nitrogen and oxygen exist in practically all naturally occurring substances of the body, many different molecules can be synthesized and used for specific imaging tasks.

This is why most of the PET installations in the world have the cyclotrons close to the PET system. It is a race against the clock, once the radioactive isotope is produced, to synthesize the radiopharmaceutical and get injected into the patient, so the PET scanner and the cyclotron should be a few minutes away from each other.

A list of the most commonly used radioisotopes for PET applications and their characteristics can be found in table 1.1.

Table 1.1: Properties of positron-emitting radionuclides.

\begin{tabular}{||l|c|c|c|c||}
\hline \hline Isotope & Half-life & $\begin{array}{c}\text { Maximum } \\
\text { positron energy } \\
\mathbf{( M e V})\end{array}$ & $\begin{array}{c}\text { Positron range } \\
\mathbf{( m m})\end{array}$ & Production method \\
\hline${ }^{11} \mathrm{C}$ & $20.3 \mathrm{~min}$ & 0.96 & 0.4 & cyclotron \\
\hline${ }^{13} \mathrm{~N}$ & $9.97 \mathrm{~min}$ & 1.19 & 0.7 & cyclotron \\
\hline${ }^{15} \mathrm{O}$ & $2.03 \mathrm{~min}$ & 1.74 & 1.1 & cyclotron \\
\hline${ }^{18} \mathrm{~F}$ & $109.8 \mathrm{~min}$ & 0.64 & 0.3 & ${ }^{62} \mathrm{Zn} /{ }^{62} \mathrm{Cu}$ generator \\
\hline${ }^{62} \mathrm{Cu}$ & $9.74 \mathrm{~min}$ & 2.93 & 2.7 & Reactor, cyclotron \\
\hline${ }^{64} \mathrm{Cu}$ & $12.7 \mathrm{hr}$ & 0.65 & 1.2 & generator \\
\hline${ }^{68} \mathrm{Ga}$ & $67.8 \mathrm{~min}$ & 1.89 & 1.2 & Reactor, cyclotron \\
\hline${ }^{76} \mathrm{Br}$ & $16.1 \mathrm{hr}$ & 1.90 & \multicolumn{2}{c}{} \\
\hline
\end{tabular}




\begin{tabular}{||l|c|c|c|c||}
\hline${ }^{124} \mathrm{I}$ & 4.17 days & 1.50 & 0.9 & Reactor, cyclotron \\
\hline${ }^{82} \mathrm{Rb}$ & $1.26 \mathrm{~min}$ & 3.15 & 2.8 & generator \\
\hline
\end{tabular}

The need for an on-site cyclotron is apparent; in addition, the short half-life requires a very rapid conversion from the isotope to the appropriate radiopharmaceutical, which is often difficult to achieve, and also requires a scheduling accuracy (availability and readiness of both patient and imaging instrument) which places an additional burden on achieving a successful study. The generator-produced isotopes listed in table 1 have so far not yielded a clinical procedure which fulfils the requirement listed above for a radiopharmaceutical and a patient population which provides significant diagnostic information not readily available with other techniques. This leaves F-18 as the most likely candidate for any widely used radiopharmaceutical; indeed dozens of F-18 labelled tracers are being tested for research studies [Stoecklin 1995].

\subsection{Radiochemical Laboratory}

In the radiochemical laboratory, takes place the synthesis of the used radiopharmaceuticals. Commonly used radiopharmaceuticals like ${ }^{18} \mathrm{~F}-\mathrm{FDG}$ and ${ }^{13} \mathrm{~N}-\mathrm{NH}$ are produced with automated techniques, commercially available. It is clear that the finally produced radiopharmaceutical must be submitted to quality control procedures prior to being injected to the patient. The quality control procedure includes a radiochemical, radioisotopic and full microbiological control.

\subsection{PET Scanner}

There are several types of PET scanners:

Full ring PET scanner, where the scintillation detectors cover a full 360o around the volume to be imaged. The advantages offered by this particular configuration are (a) optimal system sensitivity, which is necessary to obtain high counting statistics to achieve the needed physical detector resolution; (b) reduction of image artifacts due to tracer, organ 
or patient motion; and (c) absence of moving components (rotating heads), which would require further calibrations and would introduced additional variables to be considered.

partial ring PET scanner, which compared with full-ring systems, are characterized by lower overall sensitivity.

- hexagonal scanner with $\mathrm{NaI}(\mathrm{Tl})$ crystals,

- dual head $\gamma$-camera for coincidence measurements (without collimators), and, - $\quad \gamma$-camera with $511 \mathrm{keV}$ collimator.

All types of PET scanners have inferior technical characteristics (in terms of sensitivity and spatial resolution) and are also cheaper than the full ring PET scanner. The spatial resolution (FWHM) is approximately $4.5 \mathrm{~mm}$. The lower limit of spatial resolution is determined by the factors mentioned below:

The path of the positron inside the tissue until interacting with an electron. This path is not a straight line (it is actually a crooked one), but is considered to be a straight line for simplification purposes.

The two annihilation photons are not always emitted in exactly opposite directions (forming an $180^{\circ}$ angle). This results in a loss of spatial resolution by about $2 \mathrm{~mm}$.

If the two above factors are summed up, then the minimum spatial resolution is approximately $3 \mathrm{~mm}$, which is slightly less than that obtained in practice.

\subsection{Positron}

The positron was first predicted by Paul Dirac ("On the quantum theory of electron":1928). First experimental indications of an unknown particle were found in cloudchamber photographs of cosmic rays (Anderson 1932). This particle was identified later as the positron, which was thus the first antiparticle in physics. Positrons are the antiparticles of electrons. The major difference from the electrons is that positrons have a positive charge. Positrons are formed during the decay of nuclides that have an excess of protons in their nucleus compared to the number of neutrons. When decaying, these radionuclides emit a neutron $\left(\mathrm{n}^{0}\right)$, a positron $\left(\mathrm{e}^{+}\right)$, and a neutrino $\left(\nu_{\mathrm{e}}\right)$ as shown in the following equation:

$$
p^{+} \rightarrow n^{0}+e^{+}+v_{e}
$$


In this case, the daughter isotope has an atomic number one less than the parent, as shown in equation 1.2.

$$
(\mathrm{Z}, \mathrm{A}) \rightarrow(\mathrm{Z}-1, \mathrm{~A})+e^{+}+v_{e}
$$

\subsection{Annihilation Reaction}

While the neutrino escapes without interacting with the surrounding material, the positron interacts with an electron. During this process which is called annihilation, the masses of the positron and the electron are converted into two photons that travel apart in almost opposite directions (the angle of deviation is approximately $\pm 0.5^{\circ}$ ), as shown in equation 1.3 and figure 1.1.

$$
e^{+}+e^{-} \rightarrow \gamma+\gamma
$$

Since the entire masses are being transformed, each photon has now $511 \mathrm{keV}$ of energy.

In the early days of PET, annihilation radiation was considered to be less optimal for imaging because of its relatively high energy. However, later it has been demonstrated that the two annihilation photons traveling nearly co-linearly offer substantial advantages in the collimation of radiation. This property of the annihilation radiation is considered particularly effective in 3-dimensional (3D) tomographic imaging of the distribution of positron emitters.
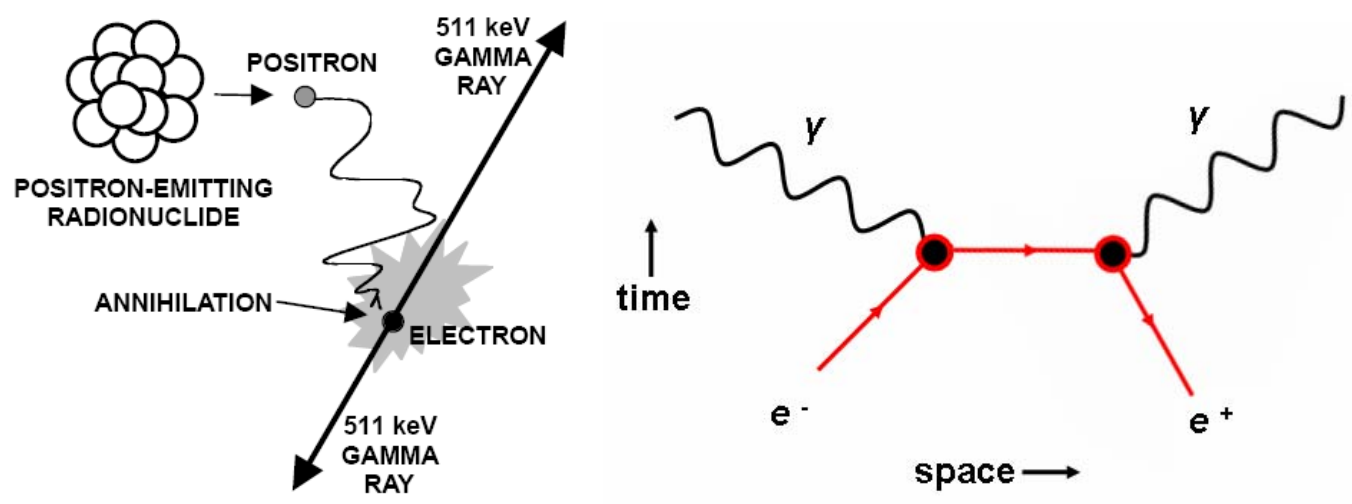

Figure 1.1: Positron emission followed by an annihilation reaction. The annihilation reaction is shown to the right in a Feynman diagram with space-time vectors. 
The annihilation of positrons with electrons can, according to quantum theory, produce high energy photons, the gamma quanta. Annihilation might take place directly or via the formation of positronium, a state in which an electron and a positron form a "light hydrogen atom" bound by Coulomb interaction. In the lowest energy states, the total spin of the system (the total sum of the two intrinsic spins) is 0 or 1 , depending on whether the spins are parallel or anti-parallel. If the annihilation occurs in the spin 0 state, the process is very fast $(<100 \mathrm{ps})$ and two gamma photons are produced and emitted in 180 degrees, each with an energy corresponding to the mass of the electron, i.e. $511 \mathrm{keV}$. The emission directions are due to the conservation of momentum. Before annihilation both the linear momentum and angular momentum are zero (if the beta particles are considered to beat rest) so the total linear momentum and angular momentum after annihilation must both be zero. This is accomplished by emission of two spin 1 photons in opposite directions. At annihilation in the spin 1 state three gamma photons are created and the available energy is shared between the three photons. Fortunately, this is a less probable process and of no interest in PET applications, since the three photons cannot easily be used to reconstruct the annihilation point.

\subsection{Detection of Photons}

The first step in order to acquire the final image is the injection of a metabolically active tracer, which is a biological molecule together with a positron-emitting isotope [Jones 1996], [Phelps 2000] (usually ${ }^{11} \mathrm{C},{ }^{13} \mathrm{~N},{ }^{15} \mathrm{O}$ or ${ }^{18} \mathrm{~F}$, although other isotopes are occasionally employed). Within a short period of time the isotope accumulates in the area of the body for which the molecule has an affinity. If for example glucose labeled with ${ }^{11} \mathrm{C}$ or a glucose analog labeled with ${ }^{18} \mathrm{~F}$ is injected into the body, then it will accumulate either in the brain or in tumors, where glucose is used as a primary source of energy. At this time the radioactive nuclei will decay by positron emission.

The ejected positron combines with an electron inside the body and these two particles annihilate. During this process the energy associated with the masses of the positron and the electron is divided equally between the two $511 \mathrm{keV}$ photons that are produced which travel in opposite directions at an $180^{\circ}$ angle. These high energy photons $(\gamma-$ 
rays) emerge from the body in opposite directions, and are detected by an array of detectors that surround the patient.

When the photons are detected simultaneously by a pair of detectors, the annihilation event that was registered to them must have occurred somewhere along the line connecting the detectors (which is called line of response (LOR)). In case that one of the photons is scattered, then the line of response will be incorrect.

\subsection{Data Corrections}

A number of corrections are typically performed on PET data both before and/or during reconstruction process. These include normalization, scatter correction, randoms correction and attenuation correction. Of these corrections, attenuation correction makes by far the biggest change in the quantitative values as well as the visual appearance of the images. This is due to the fact that attenuation can easily be a factor of 10 or greater through the center of the body. Without attenuation correction there is a significant variation in reconstructed image density across the field-of-view, with the lowest intensity in the center of the image.

Originally, attenuation correction was performed by inserting a thin, hollow cylinder of a positron emitting activity around the patient before the radiopharmaceutical was injected. This required the patient to be immobilized during the transmission scan, the radioisotope uptake period - typically $45 \mathrm{~min}$ - and the actual scan. This procedure was complicated enough to severely restrict the usefulness of the technique and most clinical whole-body scans were performed for over a decade without transmission imaging. The ability to perform post-projection transmission scans was a major step forward [Carson 1988]. This was achieved by replacing the ring of activity by a rotating line source. Since the location of the line source was known as it rotated, it was possible to differentiate between most of the emitted radiation and the transmitted radiation.

Nevertheless, the transmission scan still required as long time as the emission scan to acquire and the resulting images were quite noisy. This led to the conclusion that transmission corrected emission scans did not improve diagnostic accuracy [Bengel 1997]. 


\subsection{Attenuation of Photons}

The most important interactions which photons undergo in human tissue after positron annihilation are Compton scattering and photoelectric absorption.

In Compton scatter a proton interacts with an electron inside the tissue and during this process the kinetic energy of the electron increases while the photon changes direction. The energy of the photon after interaction is given by equation 1.4:

$$
E^{\prime}=\frac{E}{1+\left(\frac{E}{m_{0} c^{2}}\right)(1-\cos \theta)}
$$

where $E$ is the energy of the incident photon, $E$ ' is the energy of the scattered photon, $\mathrm{m}_{0} \mathrm{c}^{2}$ is the rest mass of the electron and $\theta$ is the scattering angle. Equation 1.4 implies that large deflections can occur with small energy loss - for example, for $511 \mathrm{keV}$ photons a Compton scattering event in which $10 \%$ of the photon energy is lost will result in a deflection of just over 25 degrees.

In photoelectric absorption a photon is absorbed by an atom and in the process an electron is ejected from one of its bound shells. The probability of photoelectric absorption increases as the photon energy increases. In water, the probability of photoelectric absorption decreases with roughly the $3^{\text {rd }}$ power of the photon energy and is negligible at $511 \mathrm{keV}$.

The total probability that a photon of a particular energy will undergo some kind of interaction with matter when traveling unit distance through a particular substance is called the linear attenuation coefficient $(\mu)$ of the substance. If $I_{0}$ is the initial intensity of a parallel beam of monoenergetic photons, then the intensity $I(x)$ at a distance $x$ through an attenuating object will be given by:

$$
I(x)=I_{0} e^{-\int_{0}^{x} \mu(x) d x}
$$

provided scattered photons are removed from the beam. This relation has important consequences for PET. Consider a small volume $v$ in an attenuating object, located at a distance $x$ ' along a LOR joining two detectors in the FOV of a PET camera, as shown in figure 1.2. Let the volume $v$ contain some positron emitting substance, so that there is a flux of $511 \mathrm{keV}$ photons along the line of response joining detector 1 and detector 2 . If the linear 
attenuation coefficient at a point $x$ along the LOR is $\mathrm{m}(\mathrm{x})$, and $a$ is the distance between detectors 1 and 2, the following can be stated:

Probability of a photon reaching detector 1 from $v$ is:

$$
P_{1}=e^{-\int_{0}^{x^{\prime}} \mu(x) d x}
$$

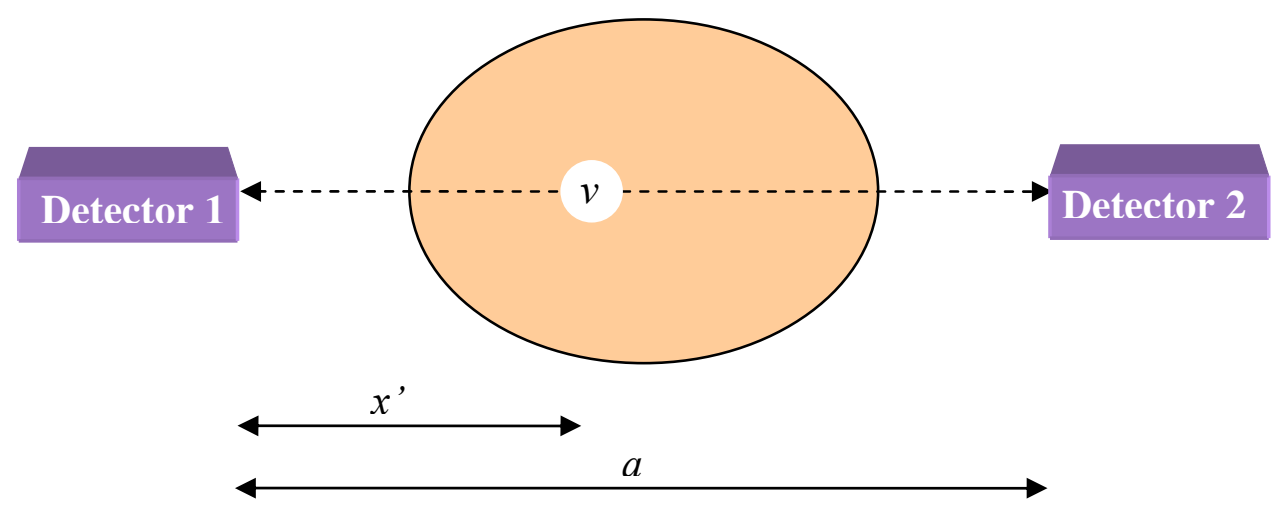

Figure 1.2: Coincidence detection in an attenuating object.

Probability of a photon reaching detector 2 from $v$ is:

$$
P_{1}=e^{-\int_{x^{\prime}}^{a} \mu(x) d x}
$$

The probabilities are independent of each other, and both photons must reach the detectors for a coincidence to be recorded. The probability of a coincidence $P_{c}$ is the product of $P_{1}$ and $P_{2}$

$$
P_{c}=P_{1} \cdot P_{2}=e^{-\int_{0}^{a} \mu(x) d x}
$$

So the quantity $1-P_{c}$ which is the attenuation factor of the photons traveling along the LOR from $\mathrm{v}$, is the same for any position along the line of response. This makes possible a simple pre-correction of data. By measuring the coincidence signal as a positron-emitting source is moved around the object within the FOV, it is possible to obtain attenuation factors for 
each LOR. In principle, this enables quantitative measurements of isotope distribution to be made.

\subsection{Scattered Photons}

Those annihilations for which one or both photons are scattered, but both are still detected, are known as scattered events. These events are incorrectly positioned because the photons' paths are not collinear. A relatively small $30^{\circ}$ scatter at the center of a typical scanner mispositions the event by $10 \mathrm{~cm}$. The overall effect is to add an error signal to the data at low spatial frequencies.

Since photons loose a fraction of their energy when they undergo a Compton interaction, they can be discriminated from unscattered photons by measuring the energy they deposit in the crystal. This can be estimated by the sum of the integrated PMT outputs. Although this measurement is only accurate to within approximately $\pm 10 \%$ on most scanners, it can be used with a simple threshold to reject a significant fraction of the scattered events.

\subsection{Accidental Coincidences}

Given the large number of scattered photons and the relatively small solid angle of the detector tin, it is apparent that for many annihilation events only one of the photons will be detected. These events are termed singles. If two singles arising from separate annihilations are detected within the same coincidence window, the will be recorded as one annihilation event. These events are termed accidental coincidences or randoms. The rate of accidental coincidences can be related to the singles rate by noting that for each single detected at detector $i$, on average $t \mathrm{R}_{j}$ singles occur at detector $\mathrm{j}$ during the coincidence timing window $\mathrm{t}$, where $R_{j}$ is the singles rate at detector $j$. Since each of these $t R_{j}$ singles results in a coincidence, there are $t R_{i} R_{j}$ coincidences per unit time for which the first detected photon is incident on the detector $i$. The total number of accidental coincidences is the sum of those for which the first photon is detected at the detector $i$ and those for which the first photon is detected at the detector $j$. Hence, the rate of random coincidences along the line-of-response connecting detectors $i$ and $j$ is given by: 


$$
R_{r}=2 t R_{i} R_{j}
$$

Equation 1.9 shows that reducing the coincidence timing window reduces the counting rate of accidental coincidences. However, timing inaccuracies, due to variations in the rise-time of the crystal light output require timing window of 10-15 ns for BGO. Since the incident singles rates are proportional to the amount of injected radioisotope, the accidental coincidence rate increases as the square amount of isotope in the field-of-view (for counting rates that do not saturate the detectors). This count rate limitation, along with the detector deadtime, determines the upper limit on the injected dose for many studies.

In figure 1.3 a true, scattered and random coincidence event are represented.
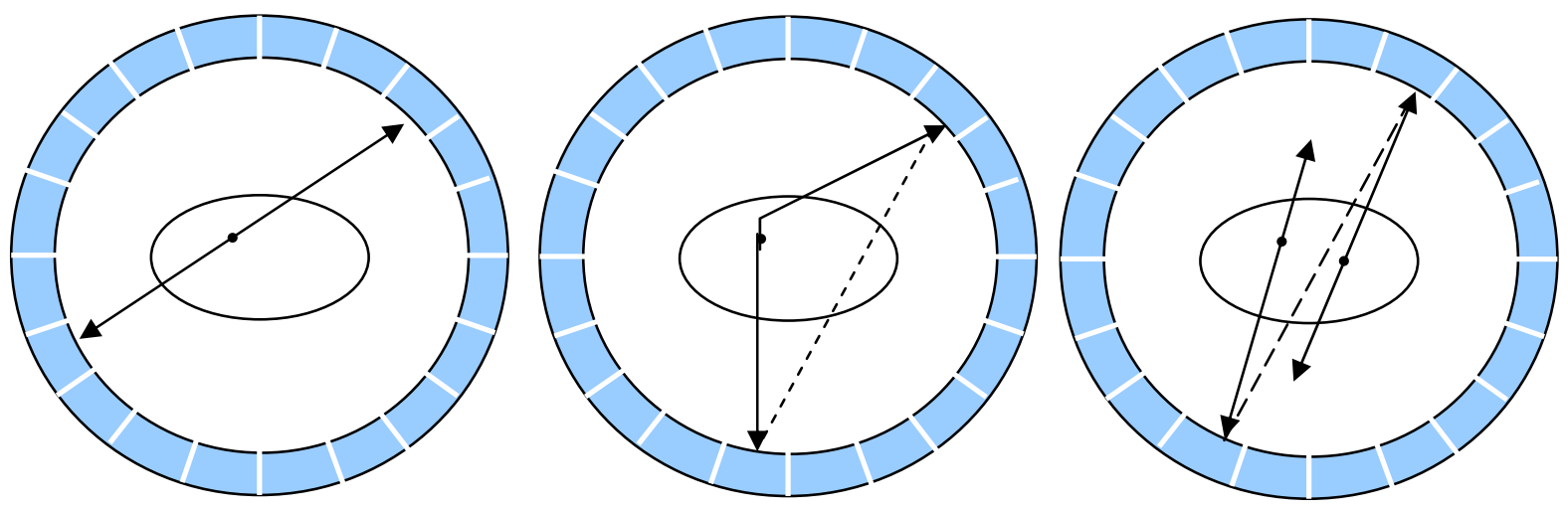

Figure 1.3: Diagram of a true event, a scattered event and an accidental coincidence. The dots represent the annihilation event; the arrows represent the gamma rays, and the dashed lines show the corresponding lines of response.

\subsection{Image Reconstruction}

After a large amount of annihilation events are detected, the distribution of the positron-emitting tracer is calculated by tomographic reconstruction procedures from the recorded projection data:

$$
P=e^{-\int a(s) d s} \int f(s) d s
$$

where $P$ is the projection data, $a(s)$ is the linear attenuation coefficient for $511 \mathrm{keV} \gamma$-rays, and $f(s)$ is the isotope distribution. The exponential factor in equation 1.10 describes the 
attenuation of the $\gamma$-rays inside the patient's body. The scattered radiation, although it might sometimes be significant is not inherent in this equation. Each two-dimensional image can be derived from the one-dimensional projections at different angles, while a threedimensional image can be derived from two-dimensional projections from multiple angles. After doing the appropriate corrections for the attenuation and scattering of $\gamma$-rays within the body, the resulting images give a quantitative measure of the isotope distribution. Therefore PET images can be used to qualitatively assess the site of unusual tracer accumulations (for example in tumors) and to quantitatively measure the tracer uptake for a more in-depth diagnosis or staging of the disease.

As the spatial resolution of PET scanners has improved, mostly by using more and smaller crystals, the number of pixels to represent the image has also increased; it is common practice to display transverse whole-body images in 128x128 format. If each slice has a thickness of $4 \mathrm{~mm}$, then a typical $100 \mathrm{~cm}$ long whole body scan has 128 x 28 x $250=4$ million pixels. Unfortunately, the number of pixels in the acquired data matrices is significantly larger (by up to two orders of magnitude), since the number of lines of response (LOR) increases proportionally to the square of the number of crystals. In fact, current clinical PET scanners typically have more LORs than the number of counts (100-200 million) acquired in a whole body scan. The reconstruction of a 3D image volume using such sparse data has been and remains a challenge.

\subsection{Clinical Applications of PET}

The clinical applications of PET include but are not limited to the following:

In Oncology, to detect recurrence of tumors earlier than with other diagnostic modalities, to detect the spread of cancer to other parts of the body from the original cancer site, to evaluate the effectiveness of cancer treatment, to assist in the management and treatment of lung cancer by staging lesions and following the progress of lesions after treatment, and to further identify lung lesions or masses detected on chest x-ray and/or chest CT.

In Cardiology, to evaluate the perfusion (blood flow) to the myocardium (heart muscle) as an aid in determining the usefulness of a therapeutic procedure to improve blood 
flow to the myocardium, to detect non-ischemic myocardiopathy, to assess the density and distribution of cardiac receptors, and to study the atherogenic risk factors.

In Neurology, to diagnose dementias (conditions that involve deterioration of mental function) such as Alzheimer's disease, as well as other neurological conditions such as:

- Parkinson's disease - a progressive disease of the nervous system in which a fine tremor, muscle weakness, and a peculiar type of gait are seen

- Huntington's disease - a hereditary disease of the nervous system which causes increasing dementia, bizarre involuntary movements, and abnormal posture,

- $\quad$ epilepsy - a brain disorder involving recurrent seizures,

- $\quad$ cerebrovascular accident (stroke).

In Psychiatry, to study schizophrenia, depression, chronic fatigue syndrome, and early deprivation.

In drug abuse research, PET scans are being used to identify the brain sites where drugs and naturally occurring neurotransmitters act, to show how quickly drugs reach and activate a neural receptor, and to determine how long drugs occupy these receptors and how long they take to leave the brain. PET is also being used to show brain changes following chronic drug abuse, during withdrawal from drugs, and while the research volunteer is experiencing drug craving. In addition, PET can be used to assess the brain effects of pharmacological and behavioral therapies for drug abuse.

In Pediatrics, for the diagnosis and staging of malignant neoplasms, assessment of epilepsy focal point, diagnosis of certain infections, and, evaluation of the therapeutic regime. 


\section{Chapter 2}

\section{Block Detectors}

\subsection{Scintillator}

A scintillator emits light when it is hit by nuclear radiation. The light is emitted from the ultraviolet to the infrared wavelength spectrum, i.e. between 100 and $800 \mathrm{~nm}$. There are several scintillating materials that are used today, such as organic crystals, organic liquids, plastics, inorganic crystals, liquids, gases and glasses. The most frequently used scintillators in PET scanners are constructed from inorganic materials. The advantage of the inorganic scintillators among all types of scintillating materials lies in their greater stopping power due to their higher density and higher atomic number Z. In addition, they have high light output, which means that a high number of photons are emitted in the visible range of wavelength. As a result of high light output, inorganic scintillators have better energy resolution, because according to statistics, the energy resolution is proportional to the square root of the number of photons. This characteristic makes them suitable for the detection of $\gamma$-ray photons. In 
table 2.1 the most frequently used inorganic scintillators are summarized, along with their properties (density, effective atomic number, etc) [Weber 2003].

Table 2.1: Properties of the most frequently used inorganic scintillators.

\begin{tabular}{|c|c|c|c|c|c|}
\hline & $\begin{array}{l}\text { Sodium } \\
\text { Iodide } \\
\text { (NaI) }\end{array}$ & $\begin{array}{l}\text { Barium } \\
\text { Fluoride } \\
\text { (BaF2) }\end{array}$ & $\begin{array}{l}\text { Bismuth } \\
\text { Germanate } \\
\text { (BGO) }\end{array}$ & $\begin{array}{l}\text { Lutetium } \\
\text { Orthosilicate } \\
\text { (LSO) }\end{array}$ & $\begin{array}{l}\text { Gadolinium } \\
\text { Silicate } \\
\text { (GSO) }\end{array}$ \\
\hline $\begin{array}{l}\text { Density } \\
\left(\mathrm{g} / \mathrm{cm}^{3}\right)\end{array}$ & 3.67 & 4.89 & 7.13 & 7.4 & 6.7 \\
\hline Effective Z & 51 & 54 & 74 & 66 & 59 \\
\hline $\begin{array}{l}\text { Relative } \\
\text { light yield }\end{array}$ & 100 & 5 & 15 & 75 & 41 \\
\hline $\begin{array}{l}\text { Decay } \\
\text { constant(ns) }\end{array}$ & 230 & 0.8 & 300 & 40 & 56 \\
\hline $\begin{array}{l}\text { Emission } \\
\text { wavelength } \\
(\mathrm{nm})\end{array}$ & 410 & 220 & 480 & 420 & 430 \\
\hline Fragile & Yes & Slight & $\mathrm{No}$ & No & $\mathrm{No}$ \\
\hline Hygroscopic & Yes & No & $\mathrm{No}$ & $\mathrm{No}$ & $\mathrm{No}$ \\
\hline
\end{tabular}

Some scintillators of table 2.1 require doping with an activator substance to obtain optimum scintillator properties. For example, $\mathrm{NaI}$ is activated by thallium (Tl), while LSO and GSO are both activated by cerium (Ce). $\mathrm{NaI}$ has been used extensively in SPECT and to a lesser extent in PET, though it is both hygroscopic and fragile. From the above table it is obvious that $\mathrm{BGO}$ has a greater stopping power compared to $\mathrm{BaF}_{2}$, which makes $\mathrm{BGO}$ more advantageous in terms of efficiency. On the other hand, $\mathrm{BaF}_{2}$ has a much faster time response which makes it more suitable for applications where good time resolution is necessary. Therefore BGO is the scintillator of choice in ordinary PET systems where there is no need for extremely good time resolution, but where the high stopping power makes it more efficient and cost effective. In time-of-flight PET (TOFPET) applications, the time 
resolution is crucial, and in this case $\mathrm{BaF} 2$ is used, which is, for the time being, the fastest known scintillator suitable for the detection of $511 \mathrm{keV}$ gamma rays. Cerium-dopped lutetium oxy-orthosilicate or LSO is a scintillator which has started to appear in the newer PET systems; however it is currently significantly more difficult to manufacture that BGO.

\subsubsection{Scintillator Requirements}

A good scintillator must comply with the following requirements:

$\square \quad$ have a large value of effective atomic number,

every $\gamma$-ray that strikes the scintillator surface should generate a large number of scintillator photons,

have a low self-absorption factor for the scintillation light,

have a refraction index close to that of glass (1.5), to improve the optical coupling between the scintillator and the photomultiplier tube.

The detectors that are used in PET applications operate at high count-rates, thus it is important for the scintillator to have a short decay time. Shorter decay times also allow faster timing signals for coincidence detection. Another factor that should also be considered is that the size of the scintillation crystal determines the intrinsic spatial resolution of the tomography. An undesirable characteristic in a scintillator is the existence of secondary scintillation components with long decay times, due to the fact that in high-count rate operation these can cause a background-light build-up. Finally, the scintillator should ideally be robust and easy to manufacture.

The emission of light after excitation and ionization in the scintillating crystal can often be expressed as:

$$
N(t)=\frac{N_{o}}{\tau_{d}} e^{-\frac{t}{\tau_{d}}}
$$

where $\mathrm{N}$ is the number of photons emitted in the visible spectrum at time t, No is the total amount of photons emitted, and $\tau \mathrm{d}$ the decay constant of emission. The rise-time has been considered to be negligible. 
One parameter that is quite important is the effect of the price of the scintillator in design. In most commercial PET scanners the cost of the scintillator material represents 30$50 \%$ of the material cost of the scanner. This severely impacts the amount of material that can be used without making the scanner prohibitively expensive. For example, $\mathrm{NaI}(\mathrm{Tl})$ is typically cheaper by a factor of 2 compared to BGO, which means that it is possible to use a $30 \mathrm{~mm}$ thick scintillator in a PET scanner with an axial field-of-view of $25 \mathrm{~cm}$, while in BGO scanners the field of view is limited up to $15-16 \mathrm{~cm}$.

\subsection{Photomultiplier Tube (PMT)}

A photomultiplier tube is a vacuum tube consisting of an input window, a photocathode, focusing electrodes, an electron multiplier and an anode usually sealed into an evacuated glass tube. In figure 2.1 the schematic of a photomultiplier tube is shown.

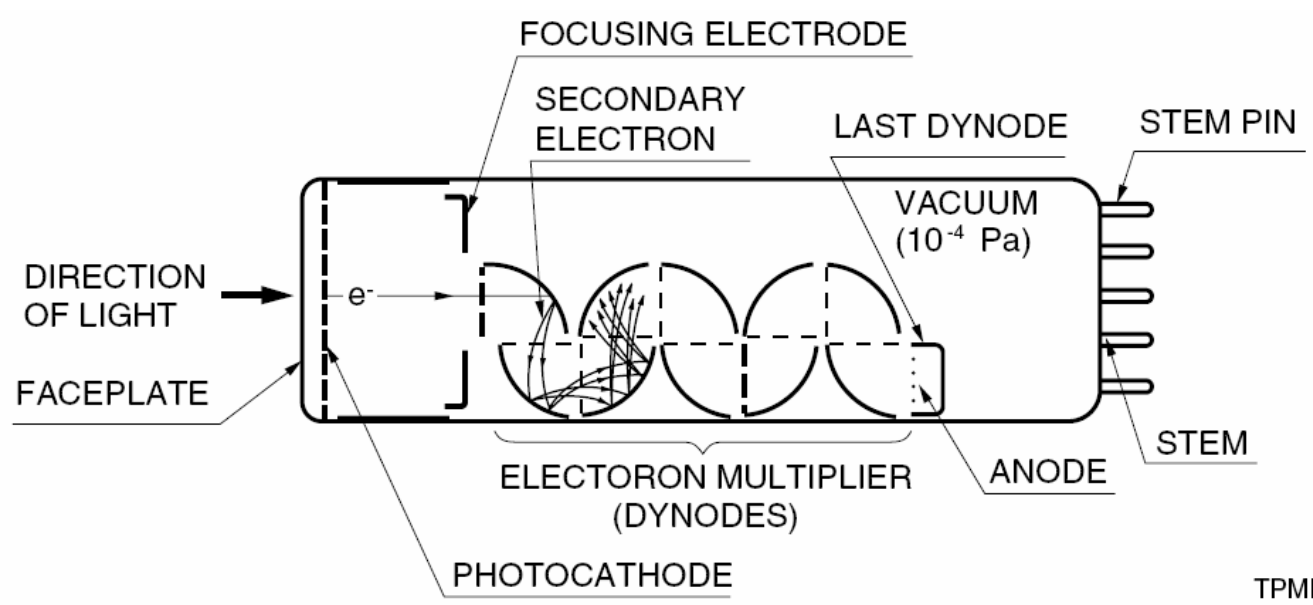

TPMHC0006EA

Figure 2.1: Cross-section of a head-on type photomultiplier tube. [Hamamatsu]

\subsubsection{Electron Trajectory}

From the moment a photon enters the photomultiplier tube until an output signal is obtained, the process is as follows:

1. A photon enters through the input window. 
2. The photon excites the electrons of the photocathode, which leads to the emission of photoelectrons into the vacuum, through photoelectric effect.

3. The emitted photoelectrons are accelerated and focused by the focusing electrode onto the first dynode where they are multiplied by means of secondary electron emission. The process of secondary electron emission is repeated in every dynode thereafter.

4. The multiplied secondary electrons emitted from the last dynode are finally collected by the anode.

The transfer of the electrons inside a photomultiplier tube is influenced by the electric field which is dominated by the electrode configuration, arrangement, and also the voltage applied to the electrode. During the process of designing a photomultiplier tube, the electron trajectory from the photocathode to the first dynode must be taken into account in order to determine the photocathode shape, which will have either a planar or a spherical window, the shape and arrangement of the focusing electrode and the supply voltage, so that the photoelectrons emitted from the photocathode are efficiently focused onto the first dynode. The collection efficiency of the first dynode is equal to the ratio of the number of electrons that are incident on the effective area of the first dynode to the number of emitted photoelectrons, which is usually better that 60 to 90\%. In PET applications where the electron transit time needs to be minimized, the electrode should be designed not only for optimum configuration but also for higher electric fields than usual.

The dynode selection is usually constructed from several to more than ten stages of secondary-emissive electrodes (dynodes) having a curved surface. To enhance the collection efficiency of each dynode and minimize the electron transit time spread, the optimum configuration and arrangement should be determined from an analysis of the electron trajectory. The arrangement of the dynodes must be designed in order to prevent ion or light feedback from the latter stages.

Of the photomultiplier tube has $n$ stages and the average number of electrons per primary electron is $\delta$ then the photoelectrons emitted from the photocathode are multiplied up to $\delta^{n}$ times and derived as a bunch of electrons from the anode. 


\subsubsection{Characteristics of Photomultiplier Tubes}

\subsubsection{Time Characteristics}

The photomultiplier tube is a photodetector that has an exceptionally fast time response. The time response is determined primarily by the transit time required for the photoelectrons emitted from the photocathode to reach the anode after being multiplied, and also from the transit time difference between each photoelectron. Thus when designing fast response photomultiplier tubes the inner window must be spherical and also the electrodes must be carefully engineered so that the transit time difference can be minimized.

The time response is primarily determined by the dynode type, but it also depends on the supply voltage. This means that if the supply voltage or electric field intensity is increased, then the electron transit speed increases, causing the transit time to decrease. The time response is related to the supply voltage by the following equation:

$$
t_{\text {resp }} \propto \frac{1}{\sqrt{V}}
$$

where $t_{\text {resp }}$ is the time response and $\mathrm{V}$ is the supply voltage.

In order to evaluate a photomultiplier tube in respect to its time characteristics we have to determine the rise and fall time, the electron transit time, the transit time spread, the cathode transit time difference and the coincident resolving time. In figure 2.2 the rise, fall and electron transit time definitions are depicted.

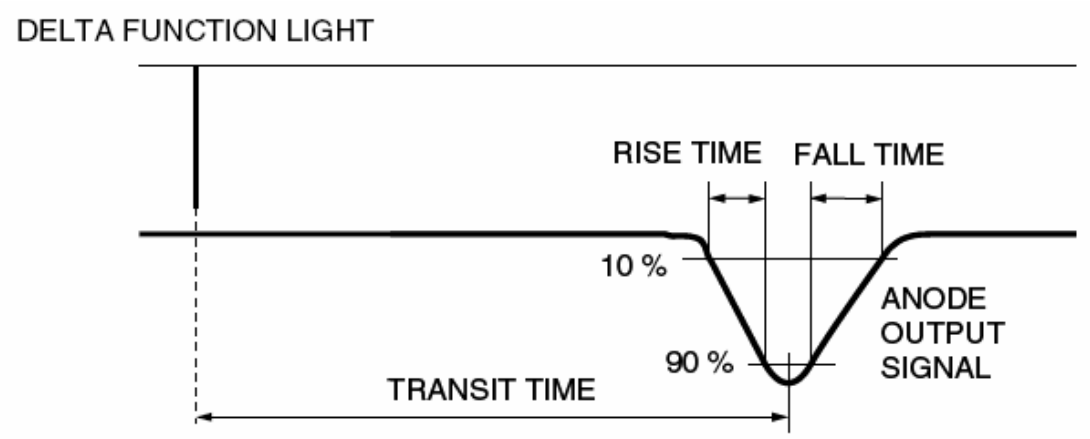

Figure 2.2: Schematic of rise, fall and electron transit time.

The rise time is defined as the time needed for the pulse to rise from $0 \%$ to $90 \%$ of peak amplitude when the entire photocathode is illuminated by a delta function light pulse (which means that the pulse's width must be less than 50ps). The electron transit time 
according to figure 2.2 is the time interval between the arrival of a delta function light pulse at the photocathode and the time where the anode output pulse reaches its peak amplitude. The electron transit time fluctuates according to the individual light pulses. This fluctuation is called transit time spread (TTS). Transit time spread is defined as the full-width-at-halfmaximum (FWHM) of the frequency distribution of electron transit times.

\subsubsection{Dark Current}

Dark current is defined as the amount of current that flows in a photomultiplier tube even when operated in a completely dark state. In order to acquire as accurate measurements as possible, dark current should be minimal. The most common causes of dark current are: (a) Thermionic emission current from the photocathode and dynodes, (b) leakage current (ohmic) leakage between the anode and other electrodes inside the tube and/or between the anode pin and other pins on the bulb stem, (c) photocurrent produced by scintillation from glass envelope or electrode supports, (d) field emission current, (e) ionization current from residual gases (ion feedback), and, (f) noise current caused by cosmic rays, radiation from isotopes contained in the glass envelopes and environmental gamma rays.

As the supply voltage increases the dark current increases too, but not at a constant rate.

\subsubsection{Afterpulsing}

When a photomultiplier tube is operated in a pulse detection mode as in scintillation counting, spurious pulses with small amplitudes may be observed. Since these pulses appear after the signal output pulse, they are called afterpulses. Afterpulses often disturb accurate measurement of low level signals following a large amplitude pulse, thus degrading the energy resolution..

There are two types of afterpulses: one is output with a very short delay (several nanoseconds to several tens of nanoseconds) after the signal pulse, and the other appears with a longer delay ranging up to several microseconds. In general, the latter pulses appearing with a long delay are commonly referred to as afterpulses. 


\subsection{Photon Counting}

One of the most sensitive and effective methods for detecting photons is scintillation counting. When ionizing radiation enters a scintillator, it produces a fluorescent flash with a short decay time. This is known as scintillation. In the case of gamma rays, this scintillation occurs as a result of excitation of the bound electrons by means of free electrons inside the scintillator. There free electrons are generated by the following three mutual interactions: the photoelectric effect, Compton effect and pair production. The probability of occurrence of these interactions depends on the type of scintillators and the energy level of the gamma rays. Figure 2.3 shows the extent of these interactions when gamma-ray energy is absorbed by a $\mathrm{NaI}(\mathrm{Tl})$ scintillator.

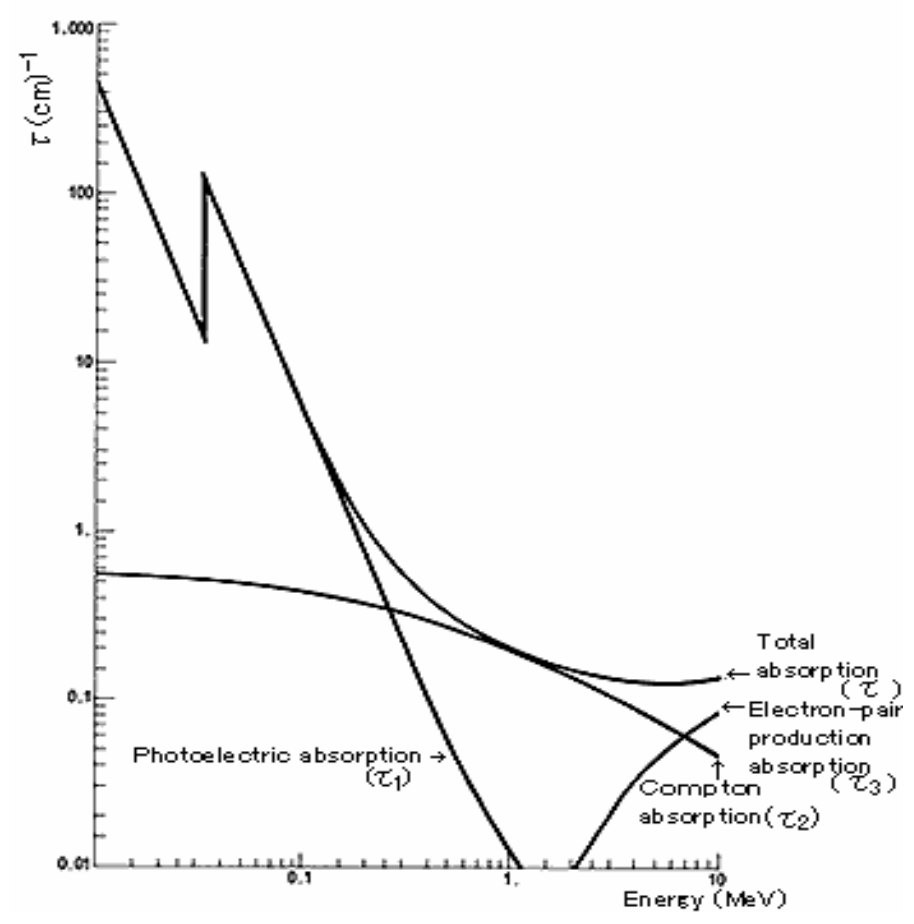

Figure 2.3: Gamma-ray absorption characteristics of $\mathrm{NaI}(\mathrm{Tl})$ scintillator.

From figure 2.3 it is clear that the photoelectric effect predominates at low energy levels of gamma rays, but pair production increases at high energy levels. Of these three interactions the amount of scintillation produced by the photoelectric effect is proportional to gamma-ray energy because all the energy of the gamma ray is given to the orbital electrons. The photomultiplier tube outputs an electrical charge in proportion to the amount 
of this scintillation; as a result, the output pulse height from the photomultiplier tube is essentially proportional to the incident radiation energy. Accordingly, a scintillation counter consisting of a scintillator and a photomultiplier tube provides accurate radiation energy distribution and its dose rate by measuring the photomultiplier tube output pulse height and count rate. To carry out energy analysis, the current output from the photomultiplier tube is converted into voltage output by an integrating preamplifier and fed to a pulse height analyzer (PHA) for the analysis of the pulse height.

A scintillator is attached to a photomultiplier tube with coupling material as shown in figure 2.4. The coupling material is used in place of an air layer in order to minimize optical loss between the scintillator and the photocathode. Silicon oil having a refraction index close to that of the glass is most widely used as a coupling material.

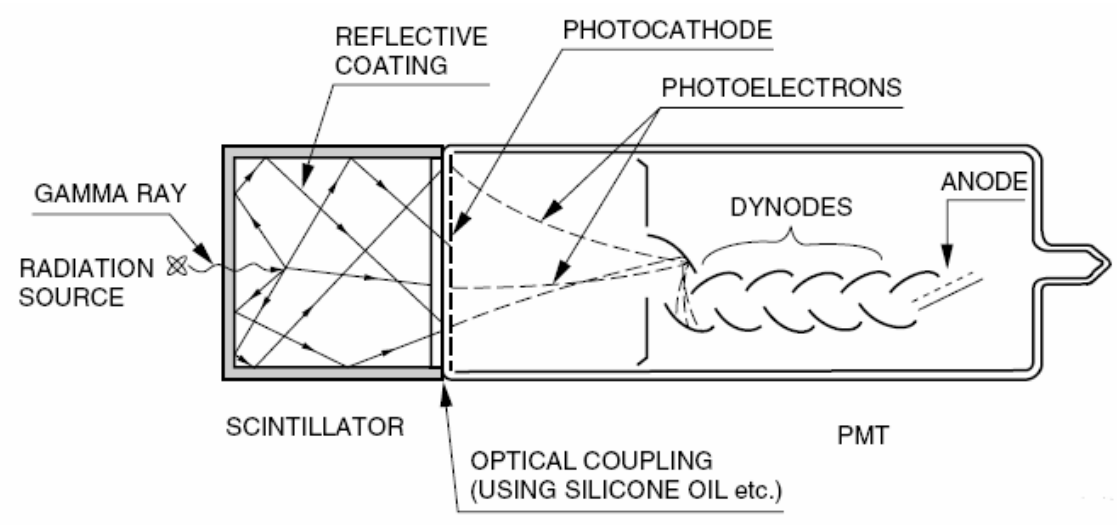

Figure 2.4: Gamma-ray detection using a scintillator and a photomultiplier tube.

\subsection{Photomultiplier Tube Output}

The output signal from a photomultiplier tube in the photon counting mode can be calculated as follows:

In the photon counting mode, a single photoelectron e- (electron charge $1.6 \times 10^{-19}$ Coulombs) is emitted from the photocathode. If the photomultiplier tube gain $\mu$ is $5 \times 10^{6}$, then the anode output charge is given by:

$$
\begin{aligned}
e \times \mu & =1.6 \times 10^{-19} \times 5 \times 10^{6}(\text { Coulombs }) \\
& =8 \times 10^{-13}
\end{aligned}
$$


Here, if the pulse width $\mathrm{t}$ (FWHM) of the anode output signal is 10ns, then the output pulse peak current Ip is:

$$
\begin{aligned}
I p & =e \times \mu \times \frac{1}{t} \\
& =\left(8 \times 10^{-13}\right)\left(10 \times 10^{-9}\right) \\
& =8 \times 10^{-5}
\end{aligned}
$$

This means that the anode output pulse width is narrower, thus we can obtain much higher output peak current. If the load resistance (input impedance of the succeeding amplifier) is 50 Ohms, the output peak voltage Vout becomes:

$$
\begin{aligned}
\text { Vout } & =I p \times 50 & & (V) \\
& =4 & & (m V)
\end{aligned}
$$

This means that the anode output in the photon counting mode is extremely small. This requires a photomultiplier tube having a high gain and an amplifier with sufficiently low noise relative to the photomultiplier tube output noise. As a general guide, photomultiplier tubes should have a gain of approximately $1 \times 10^{6}$ or more.

\subsection{Detector Characteristics}

\subsubsection{Spatial Resolution}

Spatial resolution is defined as the ability to sharply and clearly define the extent or shape of features within a PET image. It describes how close two features can be within an image and still be resolved as unique.

There are two factors that limit spatial resolution of the reconstructed images:

The angle formed between the paths of the two annihilation photons can deviate from $180^{\circ}$ as a result of some residual kinetic motion (Fermi motion) at the time of annihilation. The effect on resolution of this deviation increases as the detector ring diameter increases, so that this factor can have a significant effect. For example, for a scanner with ring diameter $80 \mathrm{~cm}$ the effect on resolution is $1.7 \mathrm{~mm}$.

The distance the positron travels after being emitted from the nucleus and before the annihilation occurs deteriorates the spatial resolution. This distance depends on 
the particular nuclide. For instance, the range of blurring for ${ }^{18} \mathrm{~F}$ is quite small (about 0.5 $\mathrm{mm}$ ) compared to that of other isotopes.

High resolution block detectors achieve a spatial resolution of approximately $4 \mathrm{~mm}$ when using ${ }^{18} \mathrm{~F}$. For events outside the central axis, the spatial resolution deteriorates significantly.

\subsubsection{Energy Resolution}

Energy resolution can be calculated by the pulse height spectrum, as the one shown in figure 2.3, using the equation 2.2. It is generally expressed as a percent:

$$
R=\frac{a}{b} \times 100
$$

where $\mathrm{R}$ is the energy resolution, $\mathrm{a}$ is the full width at half maximum and $\mathrm{b}$ is the peak value.

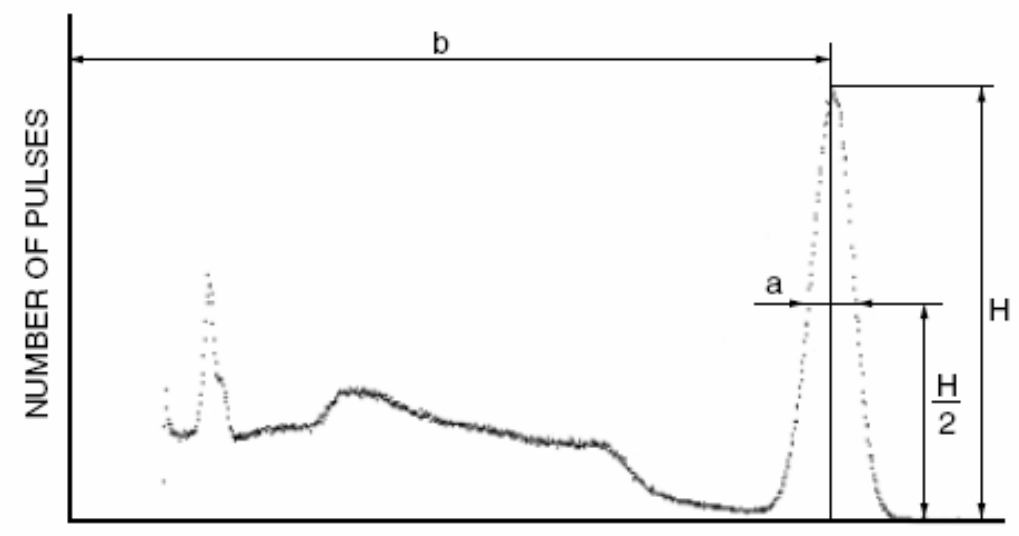

PULSE HEIGHT

Figure 2.3: Definition of the energy resolution using the pulse height spectrum.

The factors that affect the energy resolution are:

(1) The energy conversion efficiency of the scintillator, (2) the intrinsic energy resolution of the scintillator, (3) the light collection efficiency of the photomultiplier tube photocathode, (4) the quantum efficiency of the photomultiplier tube photocathode, (5) the collection efficiency at first dynode, and (6) the fluctuations in the multiplier section of the photomultiplier tube.

To obtain higher energy resolution, the photomultiplier tube must have high quantum efficiency and collection efficiency. When the scintillator is sufficiently thick, the 
intensity distribution of light entering the photomultiplier tube is always constant over the photocathode regardless of the radiation input position, so the photomultiplier tube uniformity has little effect on the energy resolution. However, if the scintillator is thin, the distribution of the light flash from the scintillator varies with the radiation input position. This may affect the energy resolution depending the on the photomultiplier tube uniformity. To avoid this problem, a light-guide is sometimes placed between the scintillator and the photomultiplier tube so that the light flash from the scintillator is diffused and allowed to enter uniformly over the photocathode. But this technique is not necessary when using a photomultiplier tube with normal uniformity.

\subsubsection{Relative Pulse Height}

In scintillation counting, when a photomultiplier tube is operated at a constant supply voltage and the amplification factor of the measuring circuit is fixed, the variation of the pulse height at a photoelectric peak is referred to as the relative pulse height (RPH) and is commonly stated in terms of the channel number. This relative pulse height indicates the variation of the pulse height obtained with a photomultiplier tube in scintillation counting. It usually shows a good correlation with measurement data and is therefore used to select the gain range in photomultiplier tubes.

\subsubsection{Uniformity}

Uniformity of a photomultiplier tube is defined as the variation in the output current with respect to the photocathode position on which a light spot is scanned. The photomultiplier tube is set at a distance (d) from a light source. The output variations of the photomultiplier tube are measured while the light source is rotated around the tube, by changing the angle $\theta$. The same procedure is repeated at different values of $d$. Then plotting the positions $(d, \theta)$ of the light source providing equal output gives a graph similar to a contour map. Uniformity data evaluated by this method is called the azimuth uniformity. 


\subsubsection{Noise}

In scintillation counting, a signal pulse is usually produced by multiple photoelectrons emitted simultaneously from the photocathode, which create a higher pulse height than most dark current pulses do. The use of a discriminator eliminates most dark current pulses with lower amplitudes. Thus, only noise pulses with higher amplitudes will be a problem in scintillation counting. To remove this type of noise pulse, the coincident counting technique is used.

Noise pulses with higher amplitude are mainly caused by radiation released from natural radioactive elements contained in concrete buildings or the atmosphere. Furthermore, scintillators and borosilicate glass used in photomultiplier tube faceplate construction might contain ${ }^{40} \mathrm{~K}$, which releases gamma rays of $1.46 \mathrm{MeV}$ that can cause highamplitude noise pulses. These noise pulses may be a significant problem especially in lowlevel radiation measurements. The most commonly applied countermeasures are: (1) the shielding of the photomultiplier tube with lead blocks, and, (2) the use of a photomultiplier tube with a so-called K-free glass containing a very small amount of potassium in its faceplate.

\subsection{Electronic Circuits}

When a photon is detected by a scintillation detector, the electrical pulse generated by the photomultiplier tube is used to produce a timing signal. This is done by passing the pulse through an analog to digital converter (ADC), which generates a digital pulse.

The pulses from the PMT are also passed through a discriminator to sort them according to pulse height. Usually there is a lower energy-level discriminator (LLD) and an upper energy-level discriminator (ULD) which may be used to reject pulses below or above particular values. The LLD can be used to discriminate against scatter, as scattered annihilation photons have lower energy than those which are unscattered. Not all scatter can be removed this way, as many scattered photons have energy quite close to $511 \mathrm{keV}$ and the energy resolution of typical detector systems is insufficient to distinguish them from unscattered photons. The ULD can be used to reject some events where more than one photon is incident on the block-detector at the same time. 
Once timing signals have been generated from each pulse, they are passed to coincidence circuits for processing. There will usually be some time difference between two timing pulses arising from a coincidence event due to the finite time resolution of the detector. In order to deal with this, the timing pulses are passed through a gate generator, which creates an electronic pulse of duration $T$. $T$ is termed coincidence resolving time of the system. These fixed-width pulses are then passed to a logic unit, which generates a pulse if there is a signal on both of its inputs at the same time. So if a timing pulse is generated on one channel at time $t$, a coincidence will be recorded if there is a timing pulse on the other channel between $t-T$ and $t+T$. The value of $T$ must be carefully chosen, because if it is too small compared to the time resolution of the detection system, true coincidences will be missed. If it is too large, then more random coincidences will be recorded without significant increase in the number of true coincidences. For a typical BGO block detector system $\mathrm{T}$ is about $12 \mathrm{~ns}$, considerably shorter than the decay time of BGO, which is about $300 \mathrm{~ns}$.

PET electronics have limits to the rate at which events may be processed. If for example light pulses from separate photon interactions overlap to a certain extent, only one pulse will be measured by the PMT. This is known as pulse pile-up. The electronics also have a finite maximum rate at which they can process data, with typical maximum rates for pulseprocessing electronics being around $1 \mathrm{MHz}$. This means that some events will be missed. These losses are known as dead-time losses. In practice, dead-time losses tend to be dominated by pile-up within the scintillation crystals. As a result, PET scanners with larger number of discrete scintillation detectors can operate at higher count-rates than those with fewer scintillation detectors.

\subsection{Requirements for the Detector System}

The requirements for the detector system are as follows:

(1) High Z material. The interaction probability and photo fraction for $511 \mathrm{keV}$ photons should be high so that gamma rays are efficiently detected and chances for multiple interactions in finely granulated detector elements are minimized.

(2) Good energy resolution. Especially for 3D PET it is essential to reduce the fraction of detected scattered gamma rays as much as possible. 
(3) Short coincidence window. Good timing is the prerequisite for random events reduction. 


\section{Chapter 3}

\section{Instrumentation}

The modules that have been used in our system are presented in this chapter.

\subsection{NIM Crate Modules}

\subsubsection{NIM Crate}

A Mech-Tronics Nuclear Model 151 NIM Instrument Bin [Mechtronics] is used to store and operate the High Voltage Power Supply, the Discriminator and the Dual Gate Generator Modules. It includes indication lights, test points, $( \pm 24, \pm 12, \pm 6)$ switch, and twelve wired connector blocks. The connector blocks each contain ten pins that supply \pm 24 , $\pm 12, \pm 6,115$ VAC, “clean" and "dirty ground". 


\subsubsection{High Voltage Power Supply}

For our experiments we have used a C.A.E.N. model N471 [CAEN], 2-fold high voltage power supply. The high voltage is delivered via a LEMO front panel connector from channel A. The polarity has been changed to negative by inverting an internal diode bridge. The output voltage range is between 0 and $8 \mathrm{kV}$. In table 3.1 the characteristics of the power supply module are summarized.

Table 3.1: Specification data for N471 high voltage power supply.

\begin{tabular}{|c|c|}
\hline Number of channels & 2 \\
\hline Output voltage & 0 to $\pm 8 \mathrm{kV}$ \\
\hline Output current & $\begin{array}{l}\text { Max } 3 \mathrm{~mA} \text { with } 0<\mathrm{V}< \pm 3 \mathrm{kV} \\
\text { Max } 1 \mathrm{~mA} \text { with } \pm 3 \mathrm{kV}<\mathrm{V}< \pm 8 \mathrm{kV}\end{array}$ \\
\hline Polarity & $\begin{array}{l}\text { Positive or negative, settable by } \\
\text { inverting an internal diode bridge }\end{array}$ \\
\hline Long term stability & $\pm 2 \mathrm{~V}$ \\
\hline Input signals & $\begin{array}{l}\text { Inhibit input TTL standard level } \\
\text { signal on } 50 \text { Ohm impedance. } \\
\text { Minimum FWHM } 60 \text { ns. }\end{array}$ \\
\hline
\end{tabular}

\subsubsection{Quad Discriminator}

In our system we use a LeCroy model 821 NIM Quad $100 \mathrm{MHz}$ Discriminator [LeCroy]. A discriminator generates precise logic pulses in response to its input exceeding a given threshold. Output pulses are of standard amplitude and of preset duration or proportional to the input rate. The threshold is a specific voltage of interest to the user.

The output of a discriminator can be used to trigger or gate associated portions of the data collection system or to generate pulses which are to be counted. It may also be integrated into a complex logic system allowing sophisticated decisions to be made in real time. 


\subsubsection{Dual Gate Generator}

In order to generate the appropriate gate a LeCroy model 222 NIM Dual Gate and Delay Generator [LeCroy] is used. The Model 222 Dual Gate and Delay Generator provides two complete delay/gate channels in a single NIM module. The 222 eliminates the common problems exhibited by other gate generators. There is negligible recovery time associated with the unit at any width setting; it may be retriggered immediately after the gate returns to its quiescent state in all ranges. Each channel of this single module can also be used to provide delays and gate outputs and to drive bin gates in its own NIM bin (LeCroy Model 1403) and several external bins. In addition, an OR input for each channel permits the gate and delay interval to be extended by an external input.

The 222 provides a range switch and a screwdriver-adjustable potentiometer to permit continuous adjustment of gate durations from less than $100 \mathrm{nsec}$ to greater than 11 seconds. A front-panel LED remains on when gate output is present, even if extended by the OR input. The approximate gate setting may be easily determined without an oscilloscope by means of the front-panel monitor point, which provides a DC voltage related to the gate duration. A conversion graph is enclosed with the unit. In addition to preset width ranges, the range switch has a "Latch" position to provide a continuous gate controllable by either the "Start" and "Stop" inputs or by the "Start" and "Stop" pushbuttons. The push-buttons permit manual operation when the full-scale switch is set on "latch", and single-shot presettable operation when the full-scale switch is in any other position.

\subsection{CAMAC Crate Modules}

\subsubsection{CAMAC Crate}

Computer Automated Measurement and Control (CAMAC) is a data handling system that is primarily used for data acquisition but CAMAC may also be used for remotely programmable trigger and logic applications. Its function is to provide a scheme to allow a wide range of modular instruments to be interfaced to a standardized backplane called a DATAWAY. The DATAWAY is then interfaced to a computer. In this way, additions to a data acquisition and control system may be made by plugging in additional modules and 
making suitable software changes. Thus, CAMAC allows information to be transferred into and out of the instrument modules.

In our system we have used a LeCroy model 1334 CAMAC Crate.

\subsubsection{Analog-to-Digital Converter}

A LeCroy model 2249W 12-channel, 11-bitintegrating-type Analog-to-Digital Converter (ADC) [LeCroy] has been used. It features excellent linearity and stability, thus allowing operation at wide gates of up to 10 usec. Thus, the 2249W ADC is compatible with CsI and $\mathrm{NaI}$ crystals. The test feature allows all $12 \mathrm{ADC}$ 's to simultaneously digitize a charge proportional to a DC level provided to a front-panel connector or patched into the CAMAC Dataway connector.

Table 3.2: CAMAC Model 2249W Analog-to-Digital Converter specifications.

\begin{tabular}{|c|c|}
\hline ADC parameter & Description \\
\hline Analog Inputs & $\begin{array}{l}12 \\
\text { Lemo type connectors }\end{array}$ \\
\hline Gain & $-0.25 \mathrm{pC} /$ count $\pm 5 \%$ \\
\hline Full Scale Range & $\sim-500 \mathrm{pC}$ (maximum count 1980) \\
\hline Integral Non-linearity & $\pm 0.05 \% \pm(0.5 p C+0.1 \%)$ \\
\hline ADC Resolution & $0.05 \%$ (1980 total counts) \\
\hline Long term stability & $\begin{array}{l}\text { Better than } 0.25 \% \text { of reading } \pm 0.5 \mathrm{pC} / \text { week (at } \\
\text { constant temperature) }\end{array}$ \\
\hline Gate Input & $\begin{array}{l}\text { One gate common to all ADCs; } \\
\text { Lemo type connector; } \\
50 \Omega \text { impedance; } \\
\text { Minimum duration, } 30 \mathrm{nsec} \text {; } \\
\text { Maximum recommended duration up to } 10 \mathrm{usec} \\
\text { Effective opening and closing times, } 5 \mathrm{nsec} \\
\text { Internal deleay, } 7 \text { nsec. }\end{array}$ \\
\hline Fast Clear & One fron panel input common to all ADCs \\
\hline
\end{tabular}




\begin{tabular}{|c|c|}
\hline & $\begin{array}{l}\text { Lemo type connector } \\
50 \Omega \text { impedance } \\
-600 \mathrm{mV} \text { or greater clears, minimum } 50 \mathrm{nsec} \\
\text { Requires additional } 2 \text { usec settling time }\end{array}$ \\
\hline Pedestal & $\begin{array}{l}\text { Adjustable over approximately } 100 \text { counts via side- } \\
\text { panel accessed trimmer capacitor. Somewhat higher for wide } \\
\text { gate. }\end{array}$ \\
\hline Digitizing time & 106 usec \\
\hline
\end{tabular}

\subsubsection{Output Register}

The Jorway model 41 12-bit Output Register [Jorway] of the system provides up to 12 bits of information to be extracted from the Dataway write lines. This information is stored and is available at the front panel as fast N.I.M. level signals, or optionally stored as power drivers. A versatile system of updating the storage registers is offered to provide flexibility of use. Data can be strobed into storage from write lines.

In addition, the register may also be selectively set and reset. In this mode the Dataway Write lines are used as enable lines which select the bits that are to respond to the function requested.

\subsubsection{Crate Controller}

The Jorway Model 73A SCSI Crate Controller [Jorway] mount on the CAMAC Crate, allows up to 7 CAMAC Crates to be controlled by a personal computer or workstation using a Small Computer System Interface (SCSI) Bus. Either 16 or 24 bit CAMAC transfers can be selected; in the latter case the CAMAC words are expanded to 32 bits for full word buffer alignment. The order of byte transmission is user selectable as high order first or low order first.

The Model 73A functions as a master controller occupying the rightmost stations in the crate. It however supports the ACB (Auxiliary Control Bus) for operation with other auxiliary controllers in the crate. The Model 73A can report CAMAC LAMs by interrupting 
the host computer using the AEN (Asynchronous Event Notification) protocol described in the SCSI specification.

The Model 73A contains a 24 bit LAM mask register, which may be enabled by a switch. When enabled, only LAMs from stations corresponding to a set mask bit will be returned by a read-L command or generate an interrupt. If the switch is off, all LAMs will be recognized. Also incorporated is a 24 bit Mailbox register which can be written or read from either the dataway or the SCSI bus to facilitate the transfer of information from multiple controllers occupying the same crate. A LAM associated with the Mailbox option may also be used to facilitate communication between processors.

The basic idea of all these modules is primarily the digitization of the signal from the PMT output via the Analog-to-Digital Converter module. The digitized signal is then delayed by the Dual Gate and Delay Generator module. At the same time a square pulse is produced by the Quad Discriminator which is driven to the ADC gate. The lower and upper time limits of the square pulse indicate the time interval during which the PMT signal will be integrated.

\subsection{Pulser}

In order to measure the response of the photomultiplier tubes we have used a Light Emitting Diode (LED) pulser. The LED emits blue light at $700 \mathrm{~nm}$ wavelength. The pulser is connected to a personal computer through an RS-232 cable. A LabView program has been developed, which enables the automatic generation of pulses of predetermined height, width, number and period. The output pulse is transferred to a LED which is in contact with an optical fiber of $1 \mathrm{~mm}$ diameter. The purpose of the optical fiber is to emit as minimum amount of light as possible and also the light emitted must be particularly localized. The other end of the optical fiber is in contact with either the crystal or the PMT surface, as described in Chapter 4. As a trigger signal a TTL pulse is used, generated by the LeCroy 222 gate generator which also provides the gate for the digitization of the PMT signals in the LeCroy 2249W ADC. 


\subsection{Block Detector}

We have used 3 detectors, one $\mathrm{NaI}(\mathrm{Tl})$ detector to test our system, and two BGO block detectors, each one consisting of a BGO scintillator coupled to a photomultiplier tube. The mechanical method for the construction of the BGO blocks is explained below, while the characteristics of the PMTs used are explained in more detail in the next chapter.

\subsubsection{BGO Block}

BGO crystal boules were grown by the top seeded Czochralski method [Tomaszewski 2002] and inspected for bubbles and cracks prior to processing. Boules were then machined on a peripheral diamond saw to produce cylinders $21 \mathrm{~mm}$ thick. The cylinders were mounted on glass plates and cut on an automatic indexing diamond saw to produce slices nominally $4 \mathrm{~mm}$ thick. The slices were cleaned and waxed on to glass optical processing tools for fine grinding and polishing of the two opposing faces to a thickness of $3.7 \mathrm{~mm}$ and then cut on annular diamond saw to produce "stick like" pieces, nominally 4 $\mathrm{mm}$ wide. These sticks were cleaned and waxed on to glass optical processing tools for fine grinding and polishing of the remaining two faces to a thickness of $3.7 \mathrm{~mm}$. After cleaning and final inspection, the polished pixels were prepared via a proprietary treatment before the row-by-row assembly process. Each pixel was cemented into position and optically isolated with $100 \mu \mathrm{m}$ thick aluminium. After being fully assembled, the end faces were ground and polished to achieve an overall thickness of $20 \mathrm{~mm}$. The encapsulation in epoxy resin completed the construction process. The BGO block is illustrated in figure 3.1.

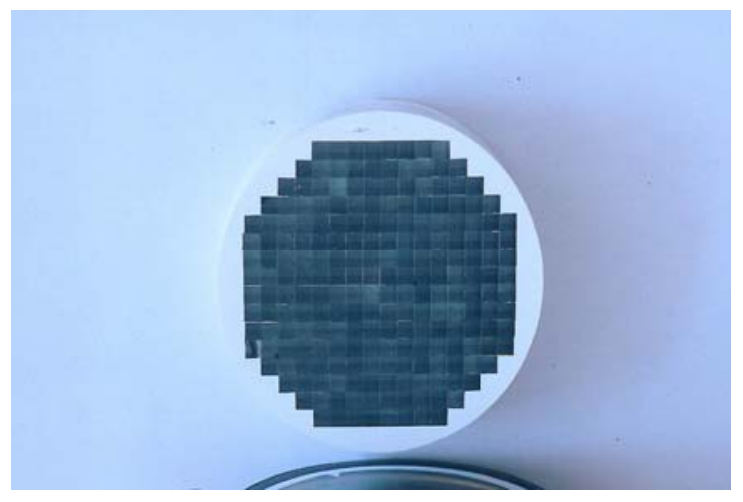

Figure 3.1: BGO crystal boules encapsulated in epoxy resin. 
The photomultiplier tubes used for the development of the block detectors will be analyzed in more detail in Chapter 4.

\subsection{Data Acquisition System}

The data acquisition and processing system contains two personal computers. The data acquisition computer (DAQ PC), which runs LabView 6.1 under Windows NT 4.0 has a SCSI (Adaptec AHA - 2490) card for the communication with the SCSI CAMAC Controller Jorway 73A. The data acquired by the data acquisition computer are transfer through the local Ethernet to another personal computer for storage and analysis with the aid of a software package based to the TCP/IP protocol. The development of this software package is based on the object oriented $\mathrm{C}++$ with built-in ROOT classes. ROOT is a software package developed at CERN for the analysis of nuclear data. A photograph of the system used is shown in figure 3.2, and the corresponding diagram is shown in figure 3.3.

According to figure 3.3, the photomultiplier tube is shielded from light inside a dark box. When the pulser emits light, a process that is controlled automatically through a LabVIEW program, the photomultiplier tube output signals are led to the ADC where they are digitized during a gate generated by the dual gate generator. The digitized data are transferred through the SCSI bus to the SCSI card of the Data Acquisition Personal Computer (DAQ PC). Through the local Ethernet the data are transferred to the Analysis Personal Computer (Analysis PC) for storage, display and further analysis. This process is automated, it is controlled by a LabVIEW [LabVIEW] program which was first used to test photomultiplier tubes for the Fermilab MINOS experiment, but we adapted the program to our needs. This LabVIEW vi (virtual instrument) control a number of parameters, and mainly the number of pulses that will be digitized when the gate will open and the number of times the gate will open. The data that are transferred to the Analysis PC are displayed in the form of spectra with the aid of a ROOT program we have written for this purpose. Another ROOT program is used for the fitting of the data with the appropriate statistical distributions. 


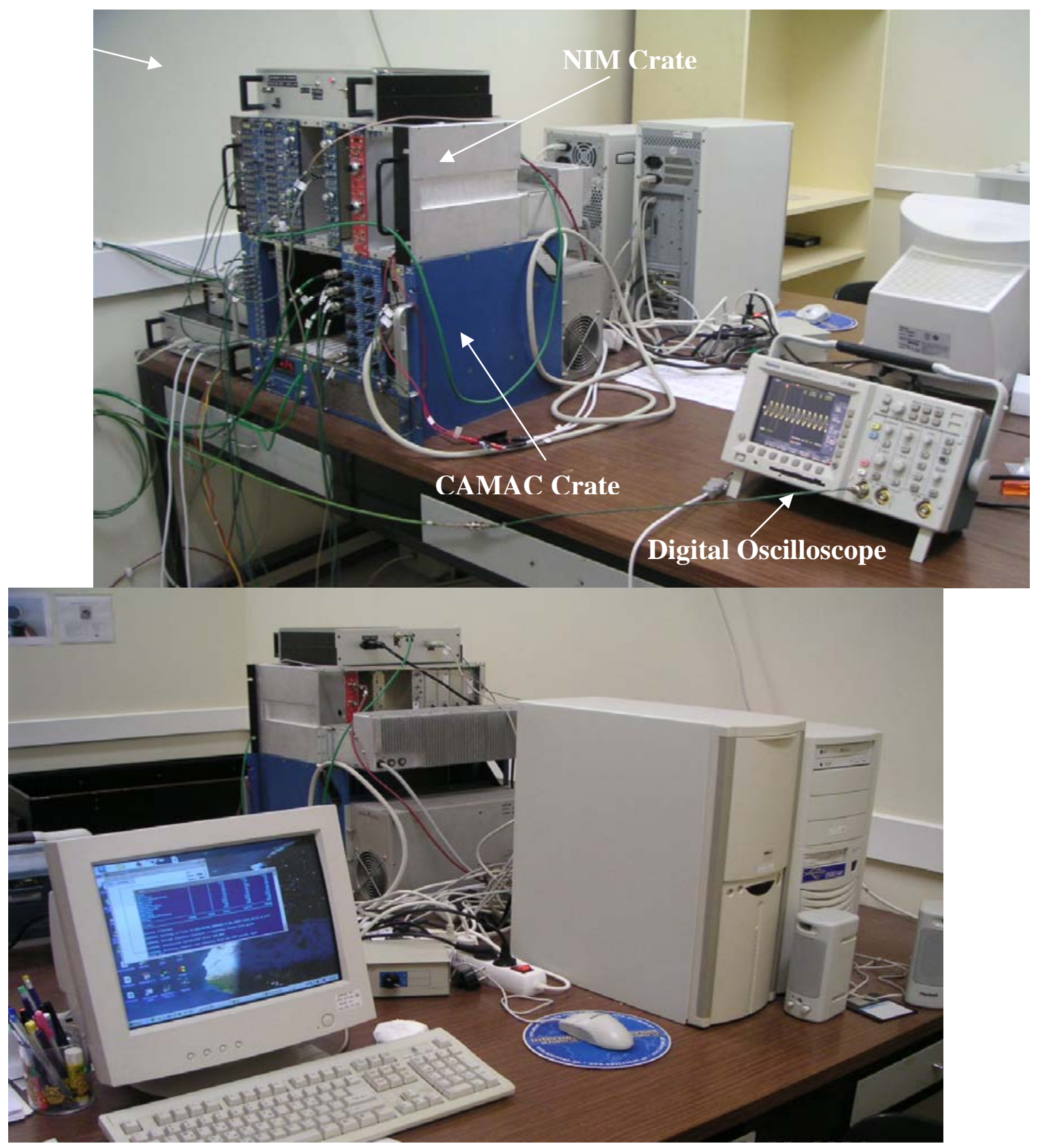

Figure 3.2: Data Acquisition system photographs. The first photograph shows the NIM and CAMAC crates along with their modules, and also the digital oscilloscope for the display of the output signals. The second photograph shows the DAQ and the analysis personal computers and also the dark box where the photomultiplier tubes are placed for testing. 


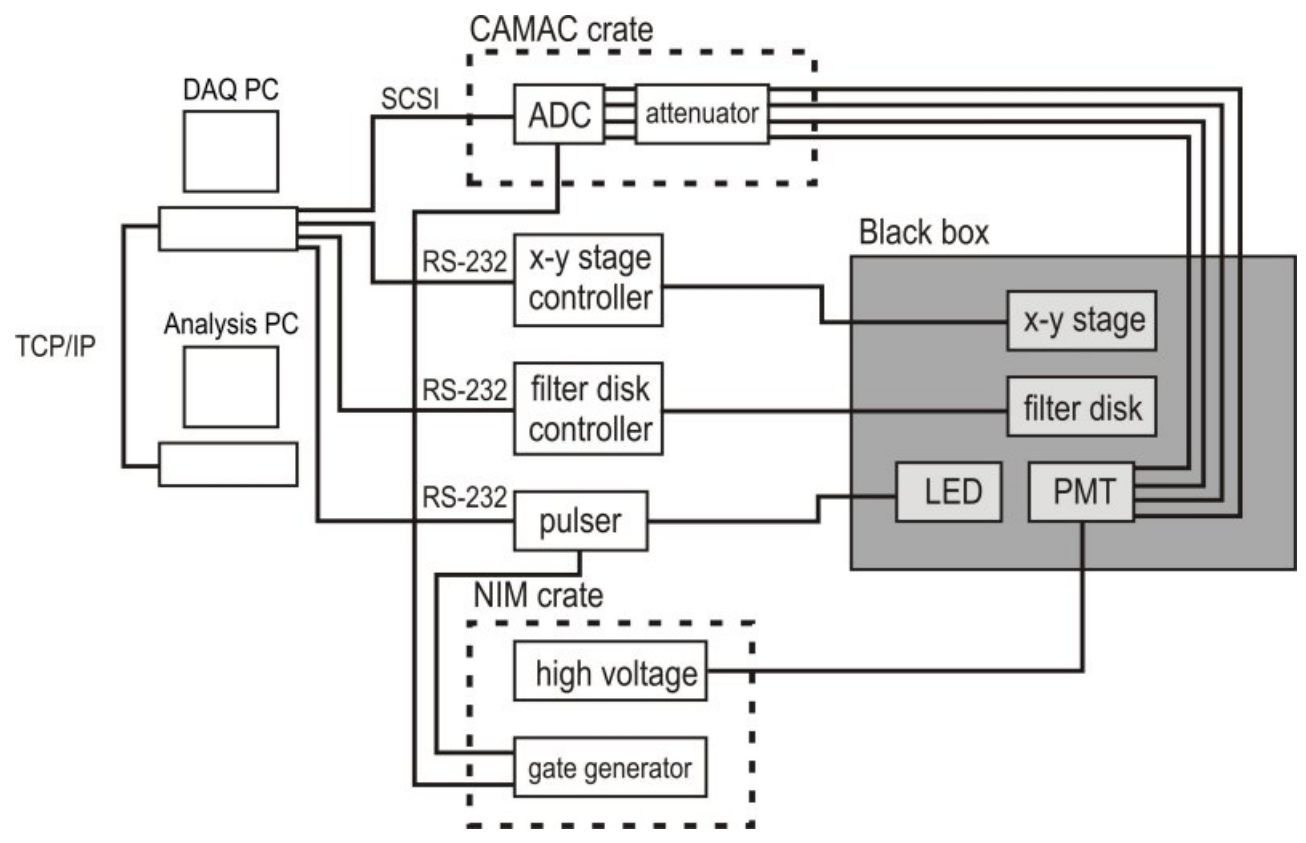

Figure 3.3: Schematic diagram of the data acquisition system. 


\section{Chapter 4}

\section{Data Acquisition}

PMT testing is one of the most demanding tasks when attempting to develop a prototype PET. This is due to the fact that the data acquired during this procedure will be used in several steps, such as the correction of the position distortion, cross-talk evaluation, gain calculations, linearity, uniformity and other measurements.

\subsection{Output Voltage Testing}

The aim of this test was to measure the actual output voltage when the high voltage power supply was set to a specific value. While gradually tuning the 10-turn potentiometer of the CAEN N471 high voltage power supply from $0 \mathrm{~V}$ to $2000 \mathrm{~V}$, we have measured the actual voltage with the aid of a polymeter (which was actually set to measure voltage). The indications of the high voltage power supply versus the indications of the polymeter are shown in figure 4.1. From the above plot it can be concluded that one quantity is a linear function of the other. 


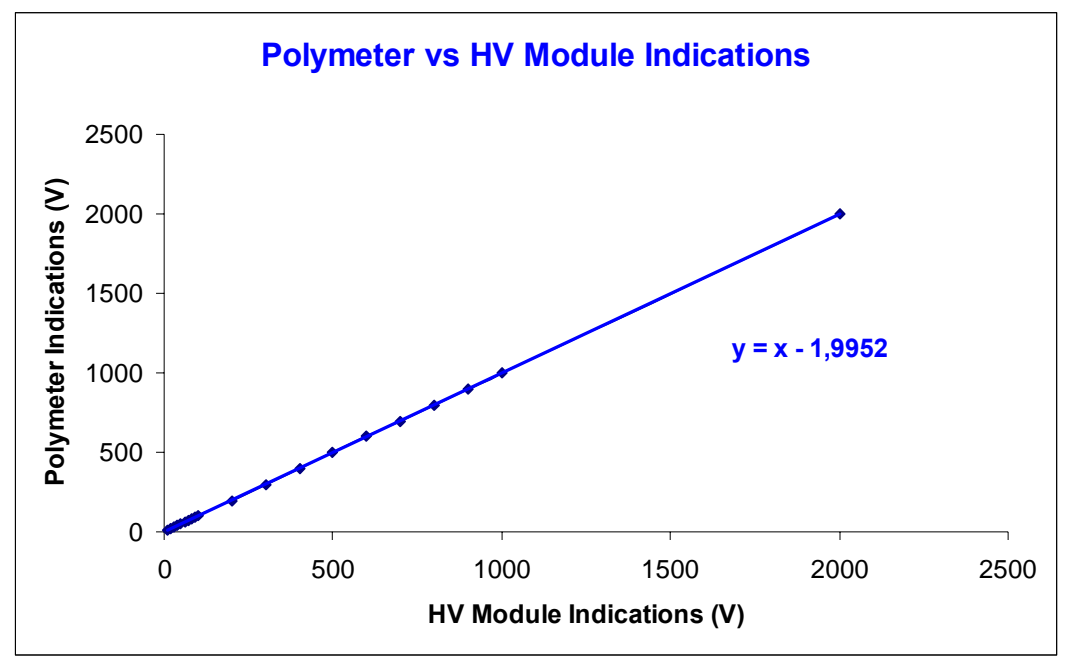

Figure 4.1: Plot of the polymeter indications as a function of the high voltage power supply indications. As it can be noticed the two indications are linearly related.

\subsection{Data Acquisition Protocol}

A specific protocol is followed for the collection and analysis of the data. In order to discriminate the two personal computers used in our system, we name the personal computer where the digitized data are transferred through the SCSI bus DAQ PC (Data Acquisition Personal Computer) and the personal computer where the data are transferred in order to be stored and analayzed Analysis PC.

The Data Acquisition Protocol steps are described below:

1. The CAMAC Crate is turned on.

2. The two personal computers are turned on. It must be checked if the SCSI card is recognized by the computer where the data are collected.

3. Then the HV power supply is turned on and is set to the standard HV value, e.g. $900 \mathrm{~V}$.

4. The pulser is turned on.

5. In the DAQ PC we check the settings in the pulser LabVIEW vi for the:

$\triangleright$ Height Top Digit: $\mathbf{a B \emptyset}$

$\triangleright$ Height Lower Digit: aC5Ø

$\triangleright$ Width: aD7, and,

$\triangleright$ Pulse multiplier: aFFF 
Alternatively, we can use the default parameters of the vi.

6. A ROOT session must be started.

7. The vi that controls the Data Acquisition system must now be opened and set the Bus Address to $\emptyset$.

8. Check if the parameters have the same values in both the ROOT file TCPIP_Connect2DAQ.C and the TCPIPClient_Jwy2249DAQ_2.vi.

The basic settings that must be the same are:

$\triangleright$ Spill size (kSpillSize)

$\triangleright$ Number of ADC channels (kNChannels)

$\triangleright$ Remote port

9. In the TCPIPClient_Jwy2249DAQ_2.vi set at the TCP/IP Root Server Address the TCP/IP address of the PC where the data are going to be transferred for processing with ROOT.

10. Run the pulser vi. In case that the pulser is not sending pulses to the detector pedestal data or dark counts can be acquired.

11. Start the ROOT TCPIP_Connect2DAQ.C

12. Run the TCPIPClient_Jwy2249DAQ_2.vi.

13. Set the Labview program priority to "High" from the Task Manager of WINNT, so that the data acquisition runs faster and the dead time is eliminated.

14. The amount of data transferred for processing is defined by the spill size. When the system acquires an amount of data equal to the spill size, ROOT creates a histogram.

With this protocol we can either digitize a photomultiplier tube signal or a pedestal. A pedestal is a photomultiplier tube output signal acquired while no light is incident on the photocathode. In other words the pedestal represents the noise of the photomultiplier tube electronic circuits. The pedestal is measured only once prior to each set of signal measurements. 


\subsection{Test PMT}

We have primarily used a test photomultiplier tube that we have borrowed from the Nuclear Physics Laboratory of the University of Athens. The basic idea for the use of such a test photomultiplier tube was to get accustomed to the whole system and the data acquisition process prior to testing the two photomultiplier tubes, mainly because the actual photomultiplier tubes to be tested are very expensive.

The test photomultiplier tube has only one output signal, as opposed to the actual photomultiplier tubes that have four output signals each as described later in this chapter.

The tests performed are described in the following sections.

\subsubsection{Photomultiplier Tube Signals}

We have used a digital oscilloscope to display the photomultiplier tube signals. A set of pulses have been registered for different values of high voltage. A plot of the photomultiplier tube pulse height as a function of the applied high voltage is shown in figure 4.2.

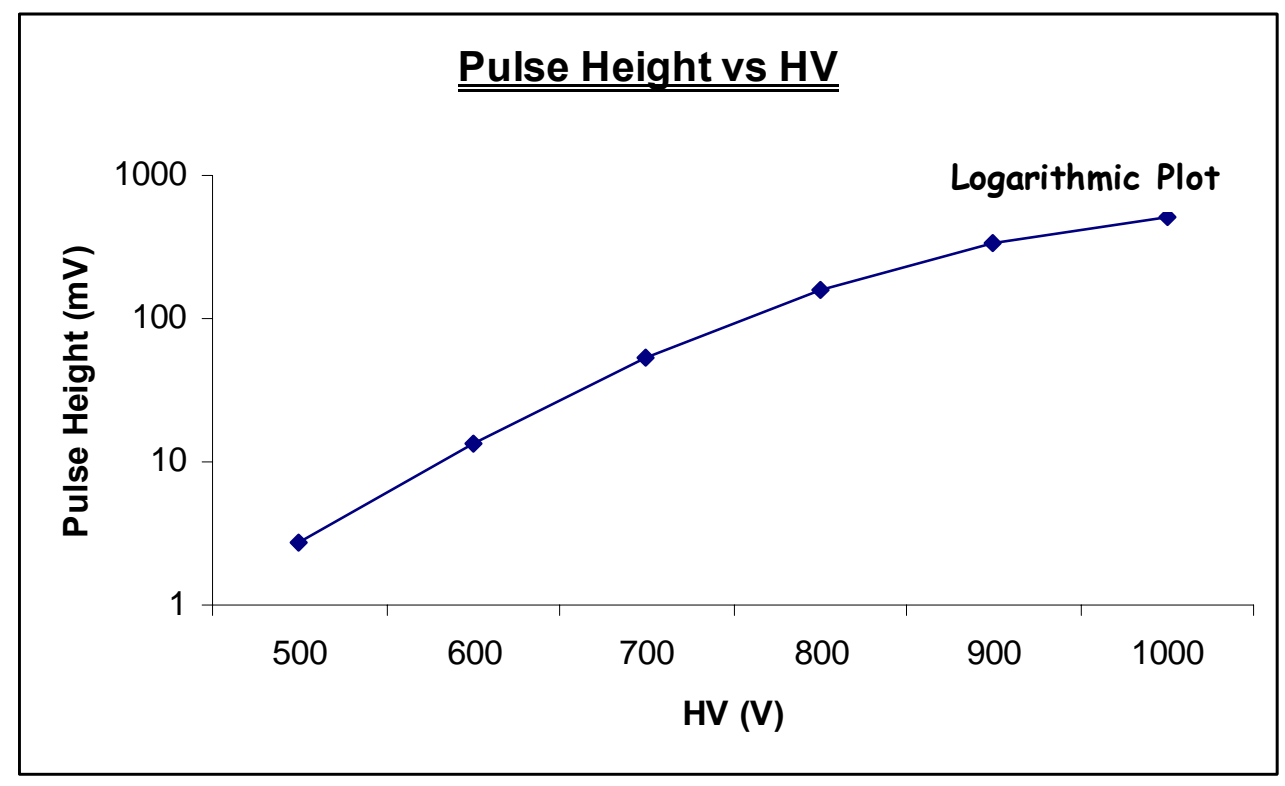

Figure 4.2: Photomultiplier tube output pulse height as a function of the applied high voltage. 
It has been observed that beginning at $-1000 \mathrm{~V}$ supply voltage, a secondary pulse appears next to the main pulse which has a width of $252 \mathrm{~ns}$ and a height of $6.6 \mathrm{mV}$. The main pulse is much higher $(520 \mathrm{mV}$ for $-1000 \mathrm{~V}$ supply voltage), but the secondary pulse is noticeable. This secondary pulse might be due to the afterpulsing effect.

The discriminator output is a square pulse. This pulse serves as the ADC gate. An example of such a pulse is shown in figure 4.3, along with the photomultiplier tube output signal. As shown in figure 4.3 the gate is set at 500 ns.

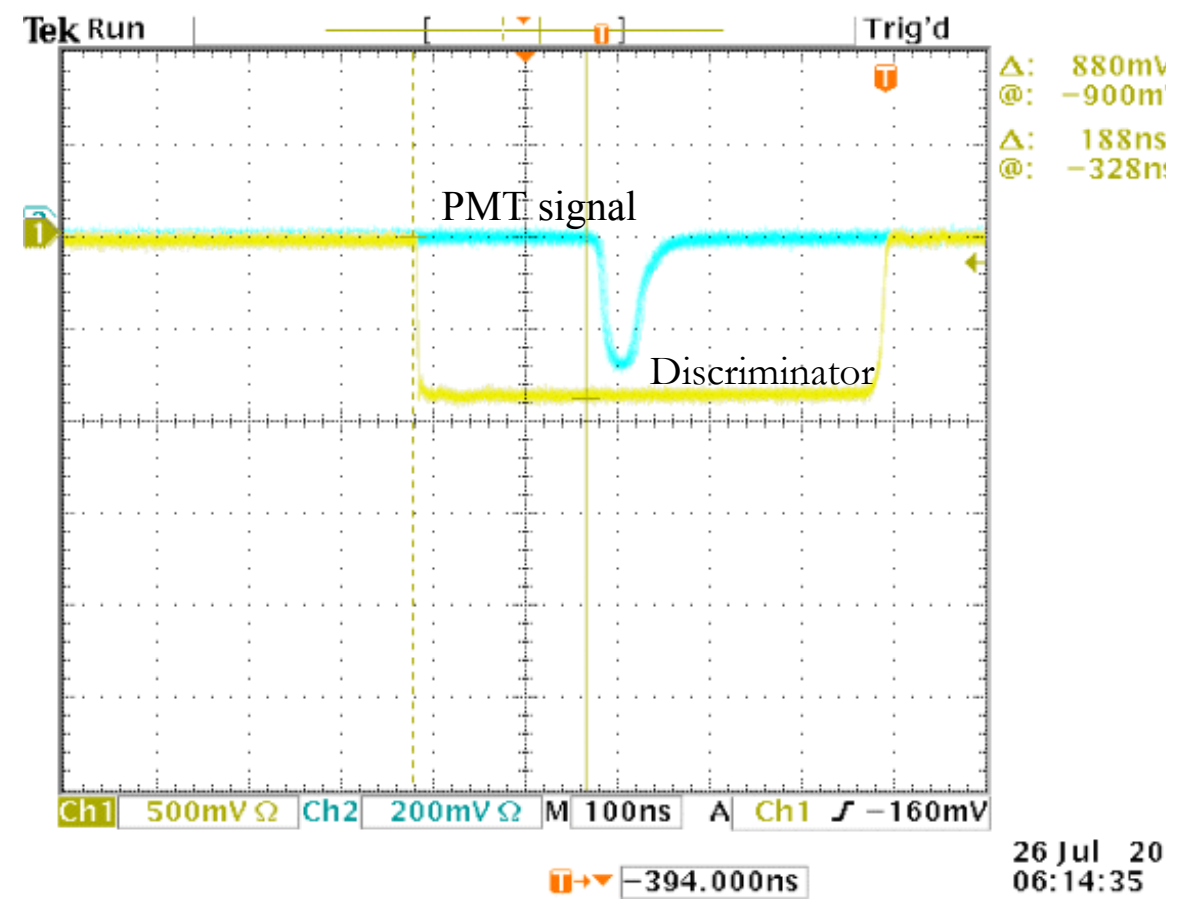

Figure 4.3: Discriminator output signal displayed together with the photomultiplier tube signal.

\subsubsection{Calculation of Photoelectrons}

Using the protocol described above, in order to calculate the photoelectrons that are emitted from the photocathode, the signal and the corresponding pedestal data should be acquired. For each photomultiplier tube signal, we obtain a digitized spectrum that can be displayed with the aid of ROOT. From the digitized spectrum, as the one shown in figure 4.4, where the $\mathrm{x}$-axis represents the number of $\mathrm{ADC}$ counts and the $\mathrm{y}$-axis represents the number of events registered in each one of the 1980 bins (according to table 3.2) of the 
2249W ADC, the mean $\mu$ and standard deviation $\sigma$ values of the corresponding Gaussian distribution can be derived. The same procedure is followed to measure the mean $\mu_{p e d}$ and standard deviation $\sigma_{\text {ped }}$ values for the pedestal. When all these parameters are known the number of photoelectrons can be calculated by the following equation 4.1.

$$
N=\frac{\left(\mu-\mu_{p e d}\right)^{2}}{\sigma^{2}-\sigma_{p e d}^{2}}
$$

For example, for the signal and pedestal shown in figure 4.4, where $\mu=1807$, $\sigma=36.51, \mu_{p e d}=123.5$ and $\sigma_{p e d}=0.047$, the number of photoelectrons $\mathrm{N}$ according to equation 4. is $\mathrm{N}=205$.

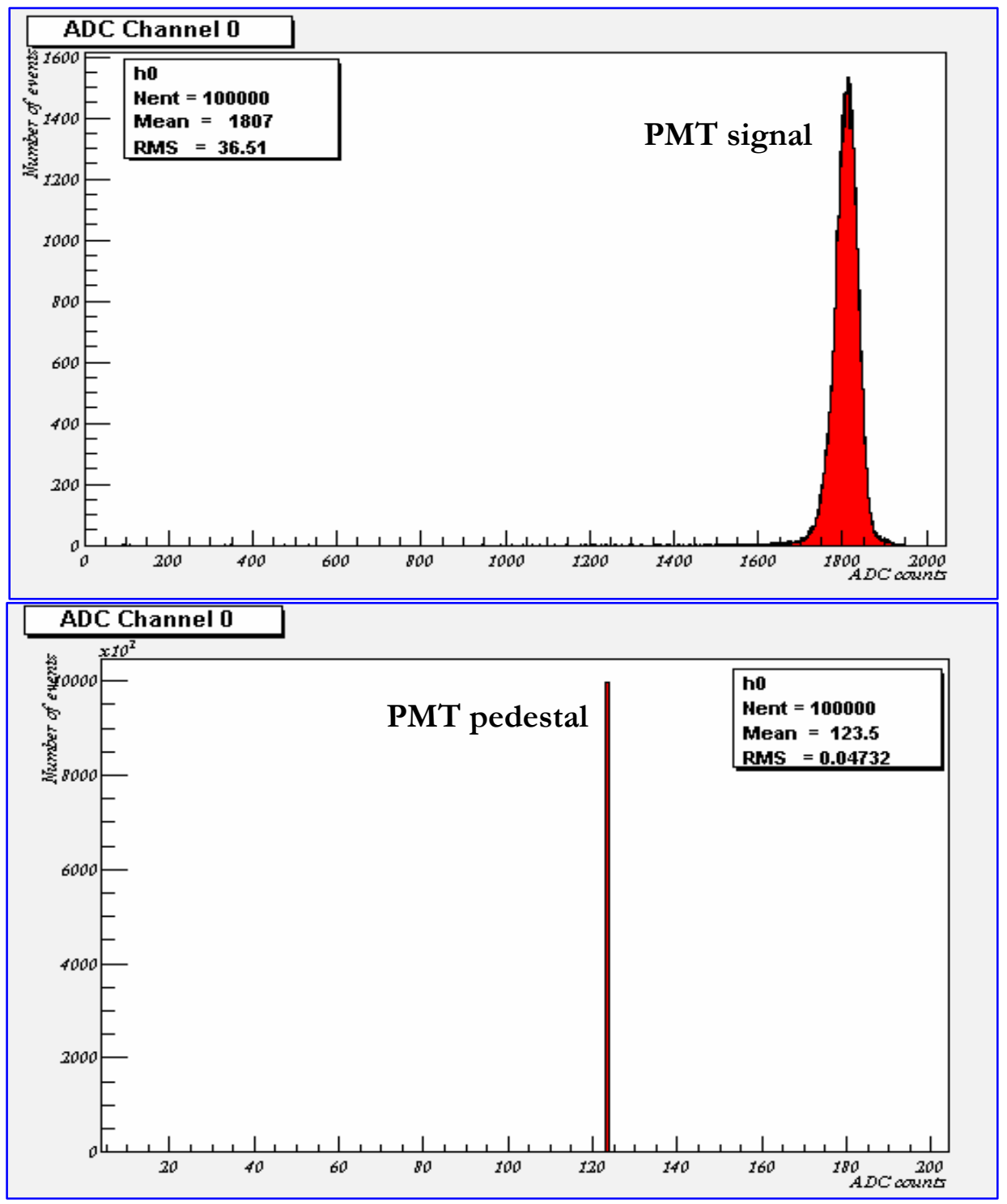


Figure 4.4: Photomultiplier tube output signal (above) and its corresponding pedestal (below).

\subsubsection{Gain Calculation}

From the mean value which is defined as the product of the number of photoelectrons $N$ (which is given by equation 4.1) by the gain of the PMT it is possible to calculate the gain G of the PMT, as follows:

$$
\mu-\mu_{p e d}=G N \Rightarrow G=\frac{\mu-\mu_{p e d}}{N} \Rightarrow G=\frac{\sigma^{2}-\sigma_{p e d}^{2}}{\mu-\mu_{p e d}}
$$

This method for calculating the number of photoelectrons and the PMT gain, referred to as the 'photon-statistics method', is not very accurate in certain points:

It does not take into account the fluctuations in the number of electrons produced at the first dynodes. Because the photomultiplier tube is shielded and also quite sensitive, these fluctuations were not measured, and subsequently not corrected.

- If the tube response is nonlinear, $\mathrm{G}$ becomes a function of $\mathrm{N}$ and equations 4.1 and 4.2 are no longer strictly valid.

\subsubsection{Dependence of ADC Counts on High Voltage}

We have studied the photomultiplier output signal while applying different values of high voltage ranging (in absolute value) from 600 to $1100 \mathrm{~V}$. The resulting log-log plot of the

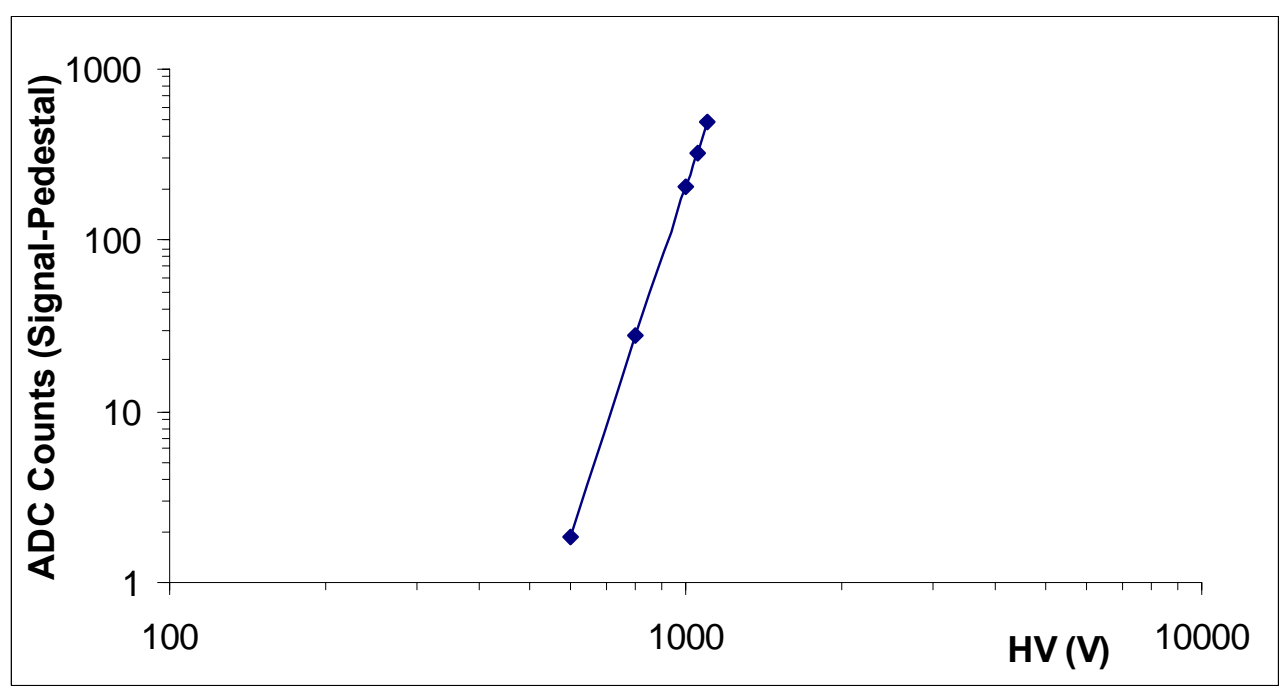


Figure 4.5: ADC counts (the ADC counts of the signal have been subtracted from the sPMT signal ADC counts) as a function of the applied high voltage.

spectrum photopeak ADC counts for different high voltage values are given in figure 4.5 from which it can be observed that the relationship between the ADC counts and the applied high voltage is a power-law, and it can be described by an equation of the form ADCCounts $=\mathrm{A} \mathrm{V}^{\lambda}$, where $\mathrm{A}$ is the slope of the straight line (in a log-log plot) measured in ADCCounts/Volt and $\mathrm{V}$ is the applied voltage.

\subsubsection{Dark Counts}

For the measurement of dark counts (see Chapter 2 for more details on dark counts), a large high voltage value must be applied on the photomultiplier tube, and at the same time no light must be incident on the PMT surface, thus the LED pulser should be disconnected. In order to measure dark counts a large amount of events must be registered, because the system must be running for quite long. In the case we are discussing, the gate is set to open 1,000,000 times, which corresponds to a DAQ system live time of about $1 \mathrm{sec}$. The corresponding dark counts spectrum is shown in figure 4.6. 


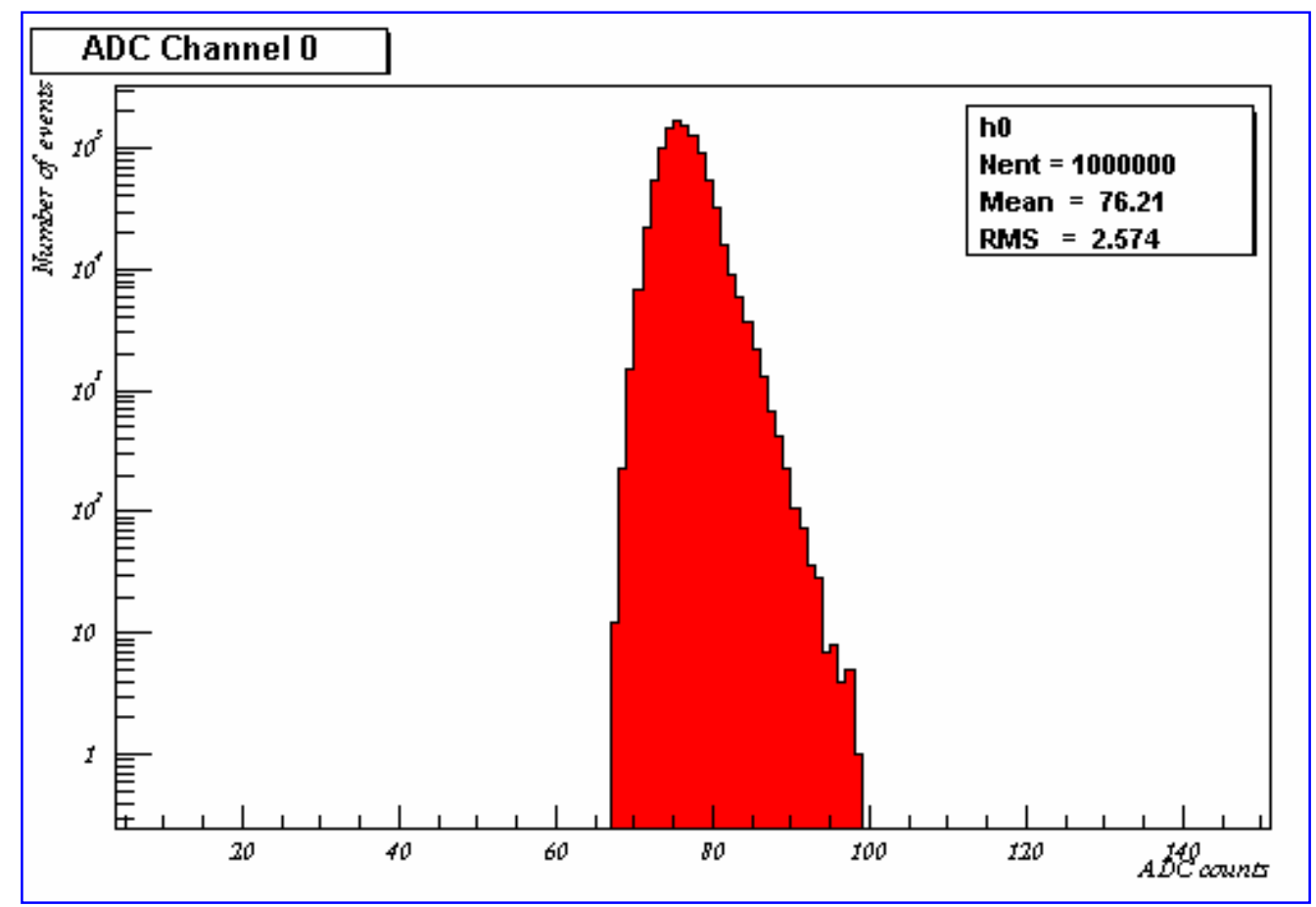

Figure 4.6: Dark counts measurement with a preset HV of $1500 \mathrm{~V}$

\subsection{System Photomultiplier Tubes Study}

We have used two Hamamatsu R2486-05 SELECT [Hamamatsu R2486] series Position - Sensitive PMTs (PS-PMTs) [Zhang 2002] with crossed wire anodes, as shown in figure 4.7. Each one consists of a bialkali photocathode, a coarse mesh dynode structure with 12 stages and an anode with 16 wires in the $\mathrm{X}$ direction and 16 wires in the $\mathrm{Y}$ direction crossing one another. Output signals from each anode can be derived through external resistive chains and derived from $\mathrm{X}$ and $\mathrm{Y}$ electrodes as the position signals, as shown in figure 4.8. More specifically, the 16 anode wires in the $\mathrm{X}$ direction end up in two analog signals $\mathrm{XA}$ and $\mathrm{XB}$, while similarly the 16 anode wires in the $\mathrm{Y}$ direction end up in two analog signals YC and YD. The advantage of the position sensitive photomultiplier tubes is that it is possible to acquire 2-dimensional information with a single tube. Until now in order to obtain 2-dimensional information an arrangement of multiple PMTs was needed, which was rather costly and required complex hardware. 


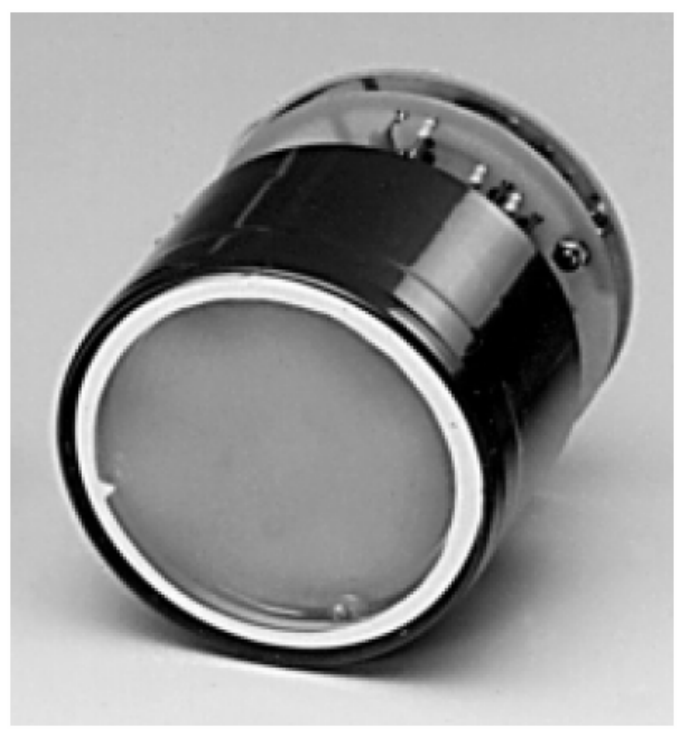

Figure 4.7: The Hamamatsu R2486-05 SELECT series photomultiplier tube.

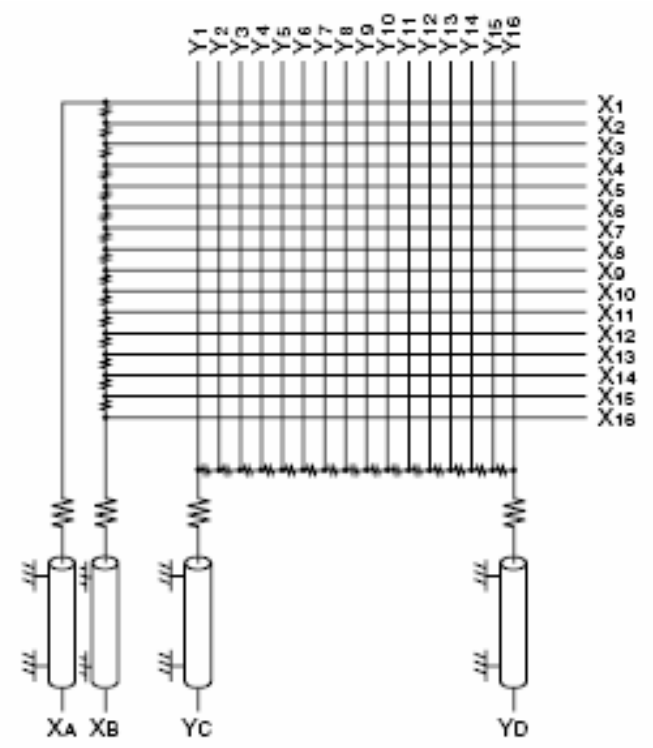

Figure 4.8: Crossed-wire anode construction.

Using the information provided by the 4 output signals $\mathrm{XA}, \mathrm{XB}, \mathrm{YC}$ and $\mathrm{YD}$ it is possible to calculate the variables $\mathrm{X}$ and $\mathrm{Y}$, according to the equations:

$$
\begin{gathered}
X=\frac{X_{A}-X_{B}}{X_{A}+X_{B}} \\
Y=\frac{Y_{C}-Y_{D}}{Y_{C}+Y_{D}}
\end{gathered}
$$


We have tested each one of the two system photomultiplier tubes separately. The characteristics of each one of the photomultiplier tubes are summarized in tables 4.1 and 4.2. For definiteness, let us name the first PMT that we have studied PMT A and the second PMT that we have studied PMT B.

Table 4.1: Characteristics of PMT A (Hamamatsu R2486-05 Serial No NA0420).

\begin{tabular}{||l|l||}
\hline \multicolumn{2}{|c||}{ PMT A } \\
\hline Parameter & Value/Description \\
\hline Type & R2486-05 SELECT \\
\hline Serial Number & NA0420 \\
\hline Cathode Luminous Sensitivity & $104.0 \mu \mathrm{A} / \mathrm{lm}$ \\
\hline Anode Luminous Sensitivity & $303.0 \mathrm{~A} / \mathrm{lm}$ \\
\hline Anode Dark Current & $6.50 \mathrm{nA}$ \\
\hline Cathode Blue Sensitivity Index & 11.40 \\
\hline Maximum Supply Voltage & $1250 \mathrm{~V}$ \\
\hline
\end{tabular}

Table 4.2: Characteristics of PMT B (Hamamatsu R2486-05 Serial No NA0406).

\begin{tabular}{||l|l||}
\hline \multicolumn{2}{|c||}{ PMT B } \\
\hline Parameter & Value/Description \\
\hline Type & R2486-05 SELECT \\
\hline Serial Number & NA0406 \\
\hline Cathode Luminous Sensitivity & $88.0 \mu \mathrm{A} / \mathrm{lm}$ \\
\hline Anode Luminous Sensitivity & $153.0 \mathrm{~A} / \mathrm{lm}$ \\
\hline Anode Dark Current & $9.60 \mathrm{nA}$ \\
\hline Cathode Blue Sensitivity Index & 10.50 \\
\hline Maximum Supply Voltage & $1250 \mathrm{~V}$ \\
\hline
\end{tabular}


Each one of the two R-2486 PS-PMTs was mounted on the test stand illustrated in figures 3.1 and 3.2. This test stand consists of a dark box where each PMT is placed while tested. Light pulses from a blue LED driven by the computer-controlled LED pulser are transported directly to the photomultiplier tube surface through an optical fiber, scanning the PMT surface with the aid of a computer controlled $x-y$ stage system on a grid of points in steps of $1 \mathrm{~mm}$. The optical fiber, blue LED and $\mathrm{x}-\mathrm{y}$ stage system are shielded inside the dark box along with the PMT. A picture of the system that is included in the dark box is shown in figure 4.9. At each fiber position, the PMT response to the incident light is recorded.

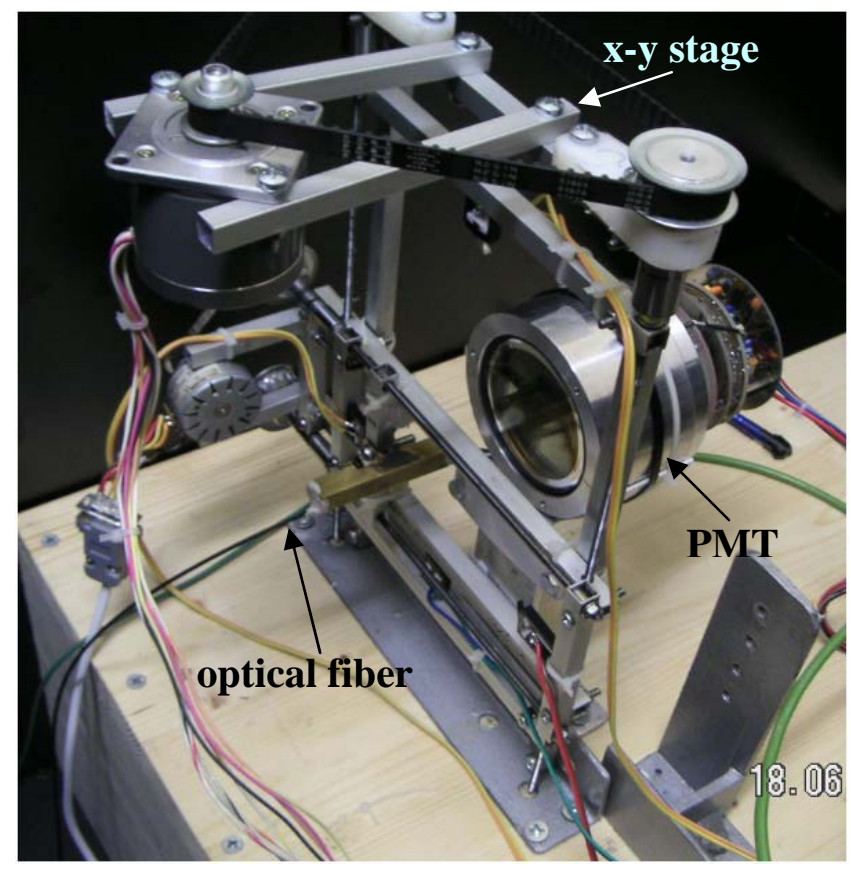

Figure 4.9: Dark box contents. The photomultiplier tube, $x-y$ stage system, and the optical fiber that extends to the photomultiplier tube surface.

The four analog signals that are generated during light emission are amplified by preamplifiers and then transferred outside the dark box, where they are integrated for a time period of $5 \mu \mathrm{s}$ and digitized using the LeCroy CAMAC model 2249W ADC. Since the dynamic range of the ADC $(500 \mathrm{pC})$ is small compared to the generated signals, the signals must be attenuated by the LeCroy Hex Attenuator Model 8102. The data acquisition system communicates with the PC with the Jorway 73A SCSI CAMAC Crate Controller. The data 
acquisition software that controls the whole process was developed using LabVIEW. At the end of the process described above, the data collected are transferred with TCP/IP communication protocol from the DAQ PC to the Analysis PC for storage, display and analysis.

The whole process is automated. When light is emitted from the LED, the optical fiber makes a stepwise movement in front of the photocathode, thus generating four signals, two in the $\mathrm{X}$ and two in the Y-direction that are subsequently integrated, digitized and read out. The analysis of the digitized signals provides us with useful information, such as the true position $(\mathrm{x}, \mathrm{y})$ of the optical fiber in relation to the calculated, using equations 4.2 and 4.3, variables $\mathrm{X}, \mathrm{Y}$. 


\section{Chapter 5}

\section{Data Processing (Results)}

With the method described in Chapter 4, we have studied both PMTs. In this chapter we present the corresponding results.

\subsection{Data Fitting}

In order to analyze the digitized signals we used a ROOT macro which calculated the parameters of the digitized signal which appears in the form of a histogram. The fitted spectra are stored in postscript files and the calculated values are registered in a data sheet.

\subsection{PMT Positioning}

Each PMT has to be positioned correctly inside the dark box before being tested. For this purpose each PMT has a guide mark -which has the form of an arrow- on its surface, which indicates that below this mark the $\mathrm{X} 1$ wire can be found and the direction of the arrow points to the last X-direction wire, i.e. X16. The axis that is vertical to the one formed by extending the arrow is the Y-Axis. A schematic of the definition of X-and YAxis is given in figure 5.1. In order to verify that each one of the PMTs was positioned 
correctly, we took a set of measurements, as the ones shown in figures 5.2 and 5.3. The idea was to scan two single lines that are perpendicular to each other. If the point where the two lines cross coincides with the point $(0,0)$ and the rest of the points are parallel to the $\mathrm{x}$ and $\mathrm{y}$-axes accordingly, then the positioning of the PMT would be correct. In our case, as shown in figures 5.2 and 5.3 the positioning is quite satisfactory.

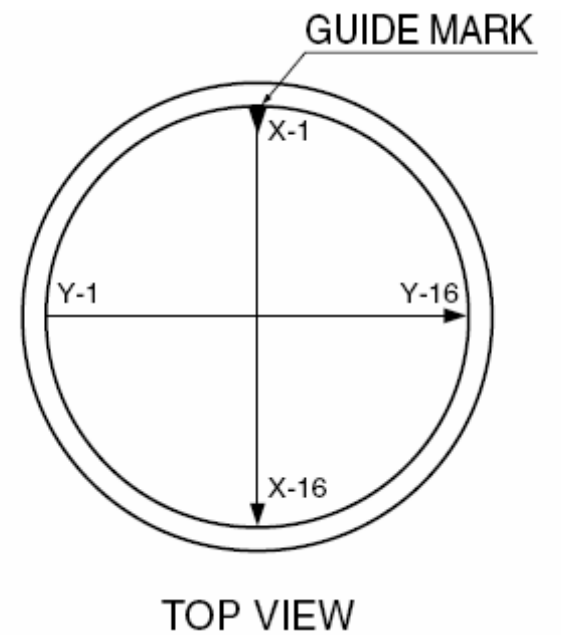

Figure 5.1: X- and Y- Axis of R2486 PMT.

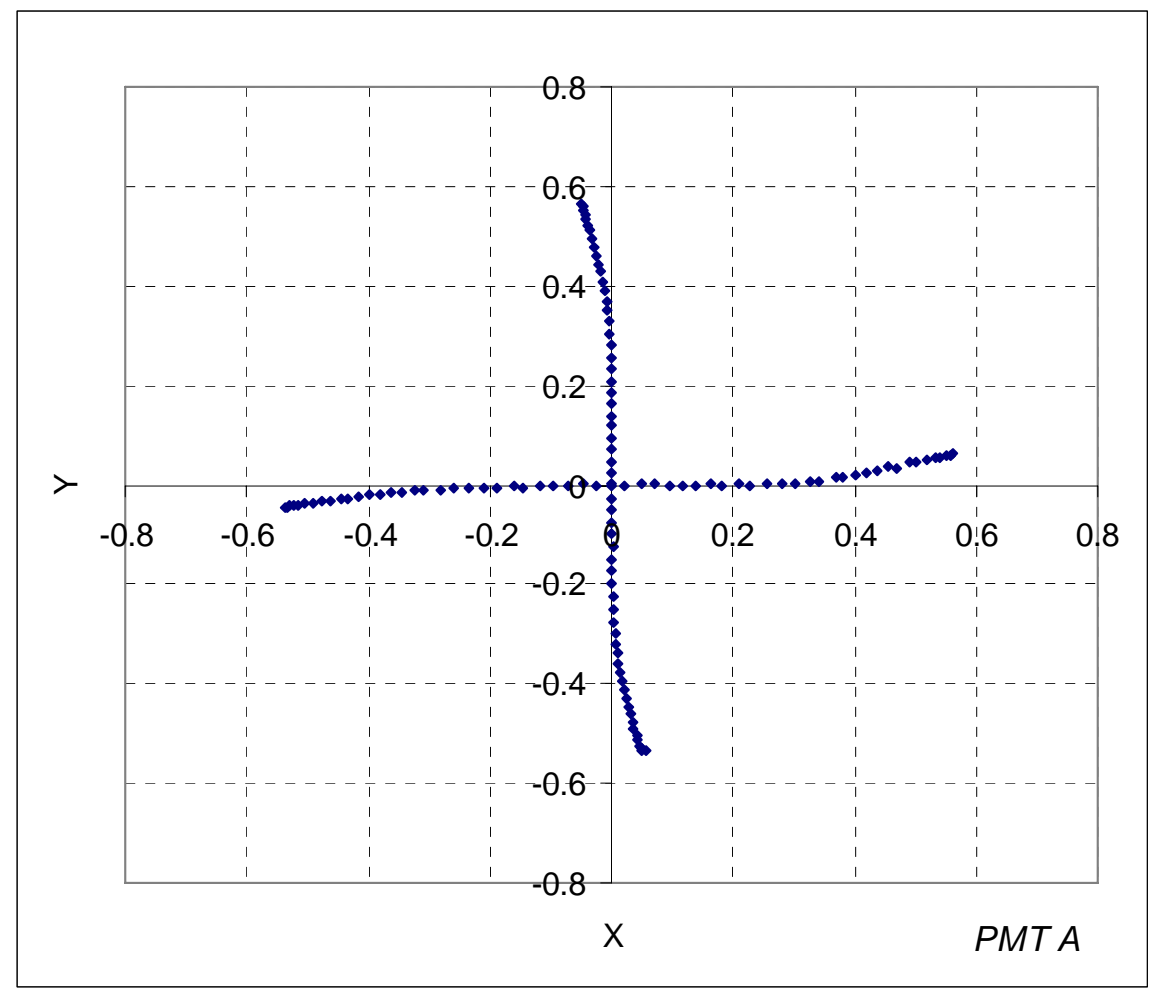

Figure 5.2: PMT A cross-like scan to determine the positioning accuracy. 


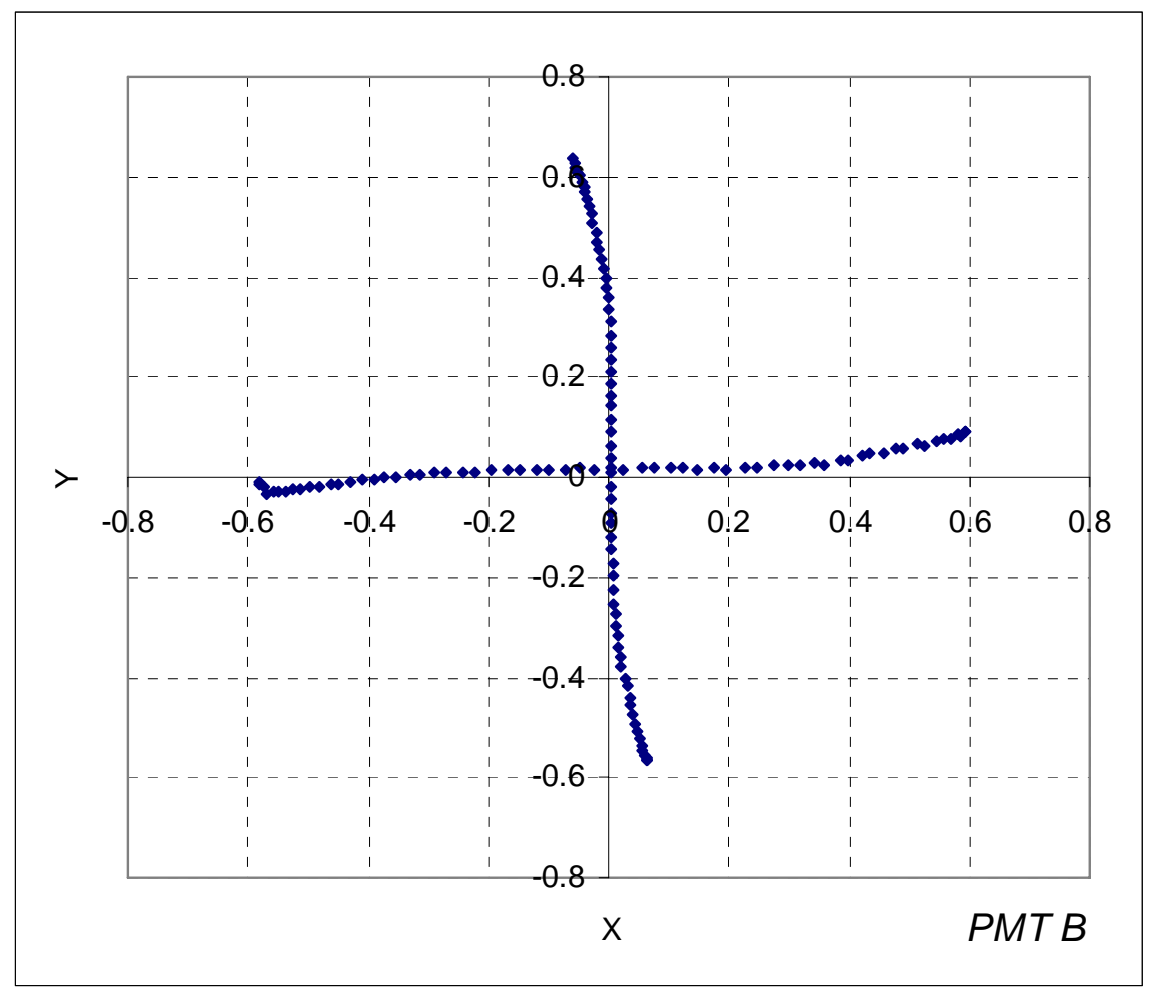

Figure 5.3: PMT B cross-like scan to determine the positioning accuracy.

\subsection{PMT Uniformity Study}

As it has been stated, each true position of the optical fiber on the PMT surface (x,y) is translated in four distinct analog signals $\mathrm{XA}, \mathrm{XB}, \mathrm{YC}$ and $\mathrm{YD}$. By performing a stepwise movement of the LED along the PMT surface following a certain horizontal line, a plot such as the one presented in figure 5.4 is generated. From this plot it is obvious that as we move away from the anode wire near XA, signal XA decreases as opposed to signal XB which increases in relation to x. Ideally, in figure 5.4 XA should increase linearly and then decrease also linearly. The opposite holds for signal $\mathrm{XB}$, as the value of the position $\mathrm{x}$ increases. The sum of $\mathrm{XA}$ and $\mathrm{XB}$ in this ideal case would be a line having three parts. In the first part the line would increase a bit more steeply than line XA, the second part would be a plateau, and the third part would be a line identical to that of the first part but with opposite slope. Such plots as the one shown in figure 5.4 are used to evaluate PMT uniformity. 
Fitting the pulse heights of all four signals using Gaussian distributions and taking the corresponding average values and finally subtracting them from the pedestals they are related to, the values of $\mathrm{XA}, \mathrm{XB}, \mathrm{YC}, \mathrm{YD}$ can be calculated. The calculation of these parameters enables us to compute $\mathrm{X}, \mathrm{Y}$ according to the equations 4.3 and 4.4 .

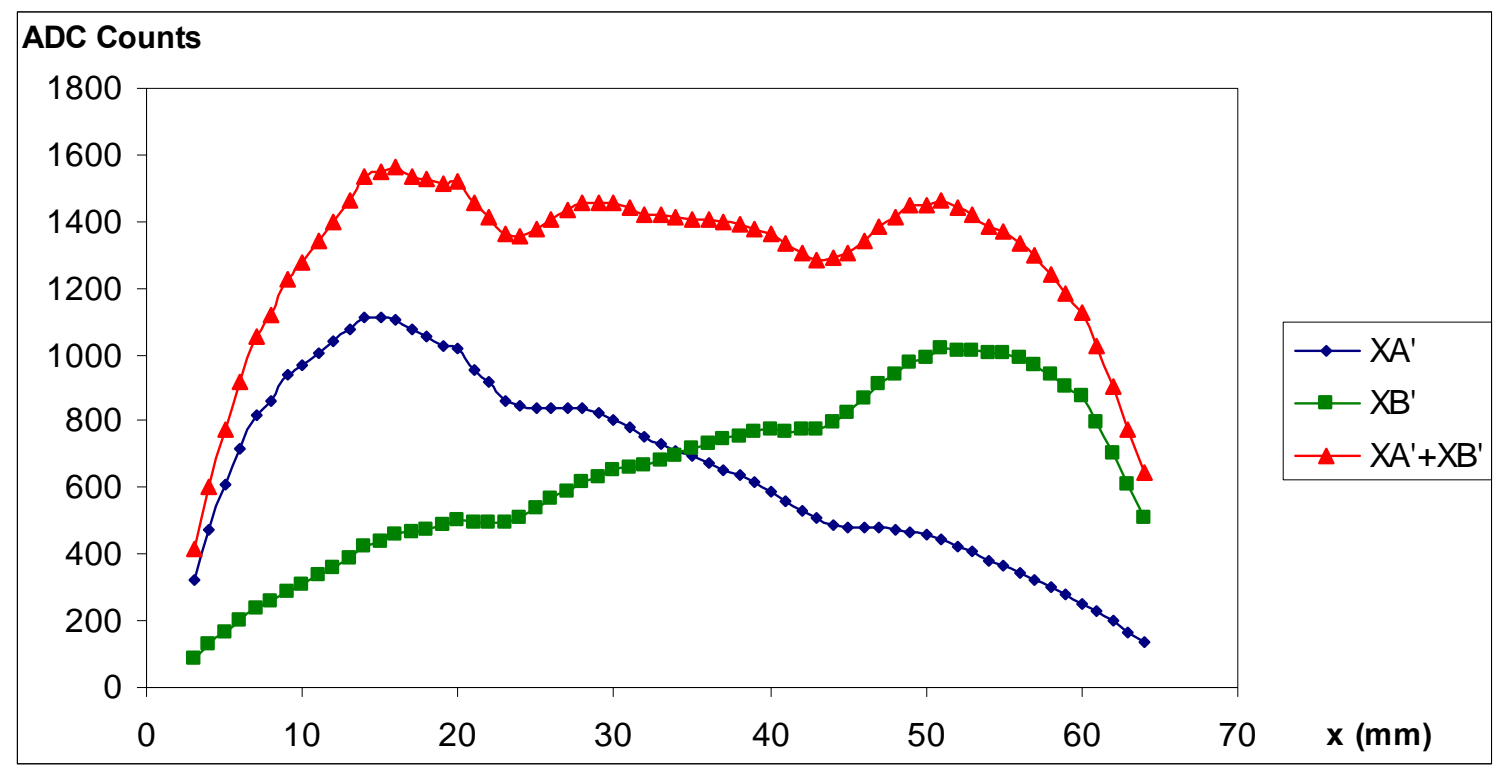

Figure 5.4: Digitized signals $\mathrm{XA}$ and $\mathrm{XB}$, and $\mathrm{XA}+\mathrm{XB}$ (with subtracted pedestals) plotted versus the real position of the optical fiber along $\mathrm{x}$-axis.

Two such plots, one for each PMT, showing the computed $\mathrm{X}$ as a function of the true $\mathrm{x}$-coordinate of the fiber, for a constant value of $\mathrm{y}$, are shown in figures 5.5 and 5.6. These plots allow us to assume a very good linearity near the center of the PMT surface. It should be noted that a deterioration of this linearity occurs near the edges, where the relationship between $\mathrm{X}$ and $\mathrm{x}$ may become non-monotonic. However we restrict ourselves to the monotonic nearly linear regime of figures 5.5 and 5.6 for the purposes of our present considerations.

\subsection{PMT Full Scan}

A complete scan of each one of the two PMTs was performed. During this scan the optical fiber was moving with a step of $1 \mathrm{~mm}$ across the PMT surface. When a line was completed across the x-axis with this step-wise movement of the optical fiber, then the 
optical fiber was shifted $1 \mathrm{~mm}$ across the y-axis. The iteration of these two movements across the $\mathrm{x}$ and $\mathrm{y}$-axis results in a square filled with dots, where each dot represents the actual optical fiber position

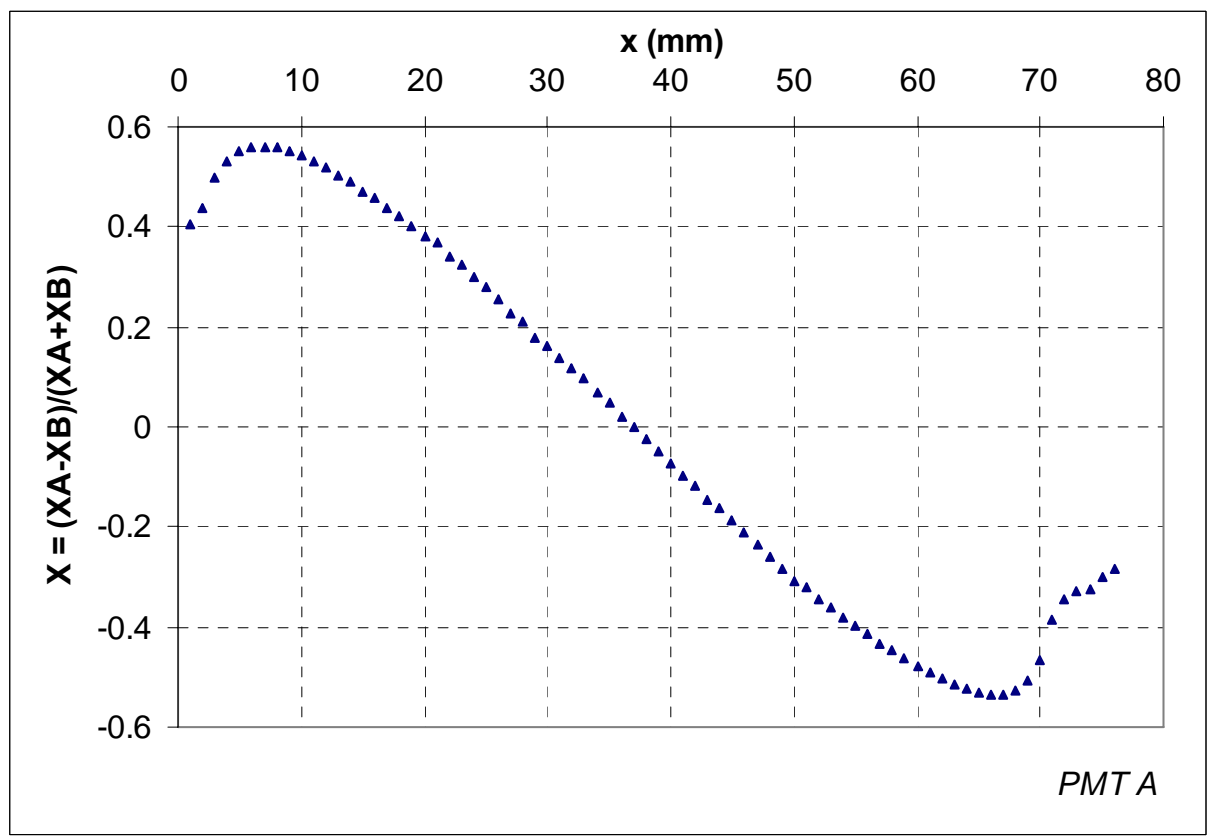

Figure 5.5: Positioning variable $\mathrm{X}$ versus the true fiber position $\mathrm{x}$ for PMT A.

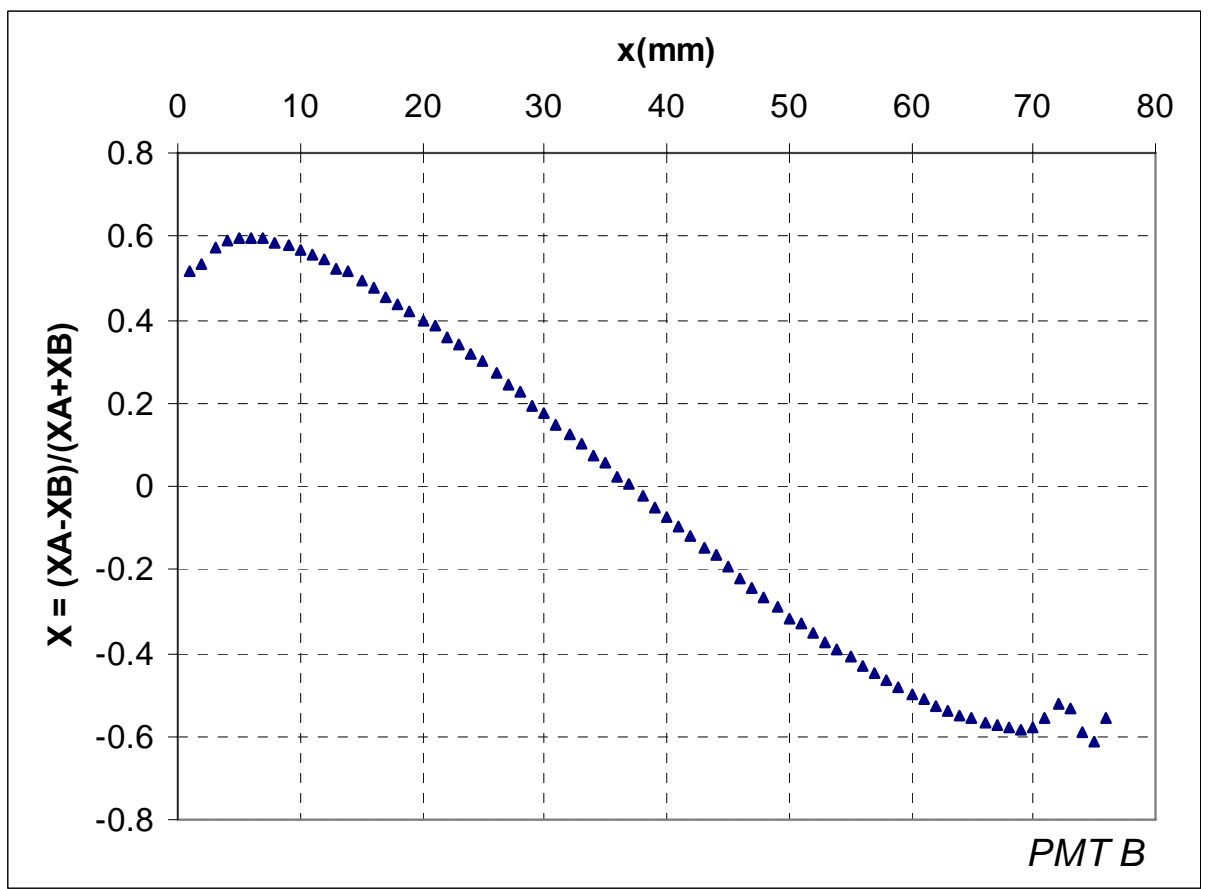


Figure 5.6: Positioning variable $X$ versus the true fiber position $x$ for PMT B.

across the PMT surface. Each true position $(\mathrm{x}, \mathrm{y})$ of the optical fiber on the PMT surface is translated in four distinct analog signals. Using the measured values of XA, XB, YC and YD the variables X, Y are computed.. Figures 5.7 and 5.9 show the map of the variable pairs $(\mathrm{X}, \mathrm{Y})$ that correspond to the true $(\mathrm{x}, \mathrm{y})$ map. The figures show clearly that there is a distortion near the edges of the PMT, where the relationship between $\mathrm{X}$ and $\mathrm{x}$ becomes nonlinear and non-monotonic, as explained in the previous section. However, the PMT is fairly linear in the central region. We have applied several criteria in our effort to eliminate inaccurate points from the figures 5.7 and 5.9. The most important such criteria were the calculation of the ratio $\chi$-square over the number of degrees of freedom for each spectrum to be below an upper bound (a non-dimensional number) and also the restriction of the mean ADC counts to be below a certain multiple of the spectrum $\sigma$ (RMS) value. By applying those two criteria figures 5.8 and 5.10 are generated. From these figures it can be observed that a large amount of mean values outside the imaginary circular PMT surface have been omitted. By inverting the relationships between $(\mathrm{X}, \mathrm{Y})$ and $(\mathrm{x}, \mathrm{y})$, it is possible to derive the functions $\mathrm{x}=\mathrm{x}(\mathrm{X}, \mathrm{Y})$ and $\mathrm{y}=\mathrm{y}(\mathrm{X}, \mathrm{Y})$. In figures 5.11 and $5.12, \mathrm{x}(\mathrm{mm})$ is plotted as a function of $\mathrm{y}(\mathrm{mm})$. 


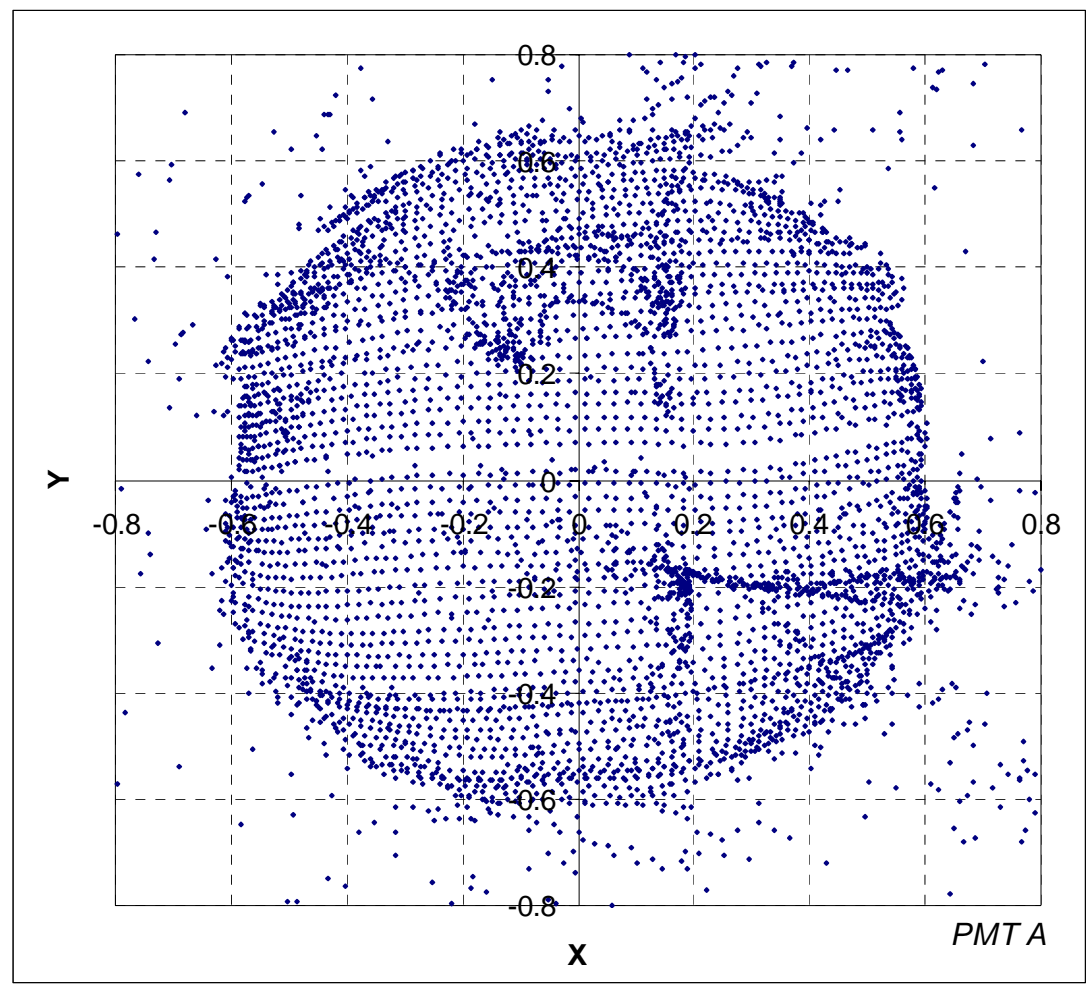

Figure 5.7: $\mathrm{X}$ versus $\mathrm{Y}$ plot in a full PMT A scan.

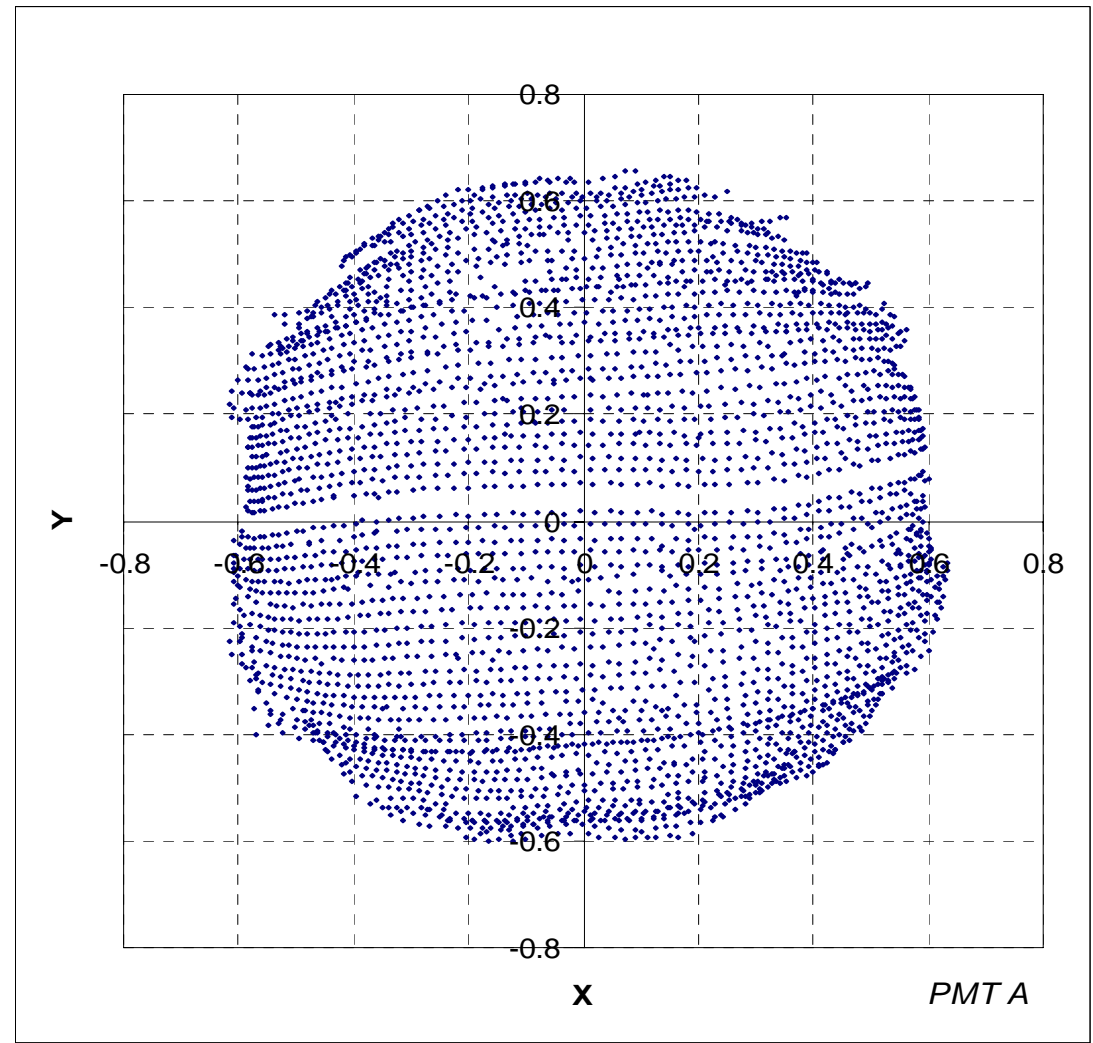


Figure 5.8: $\mathrm{X}$ versus $\mathrm{Y}$ plot in a full PMT A scan. In this case, several points have been omitted using specific criteria concerning the $\chi$-square, the number of degrees of freedom and the mean value of each digitized spectrum.



Figure 5.9: $\mathrm{X}$ versus $\mathrm{Y}$ plot in a full PMT B scan. 


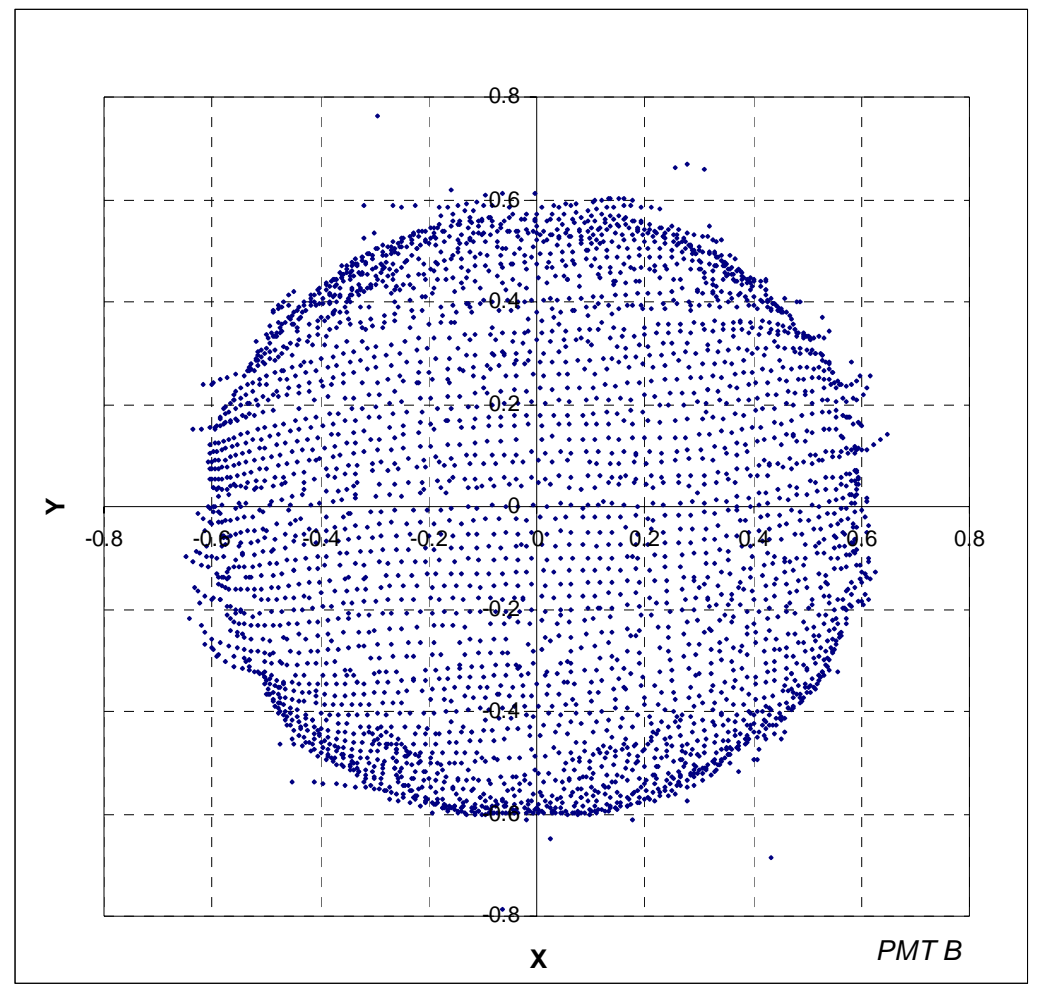

Figure 5.10: $\mathrm{X}$ versus $\mathrm{Y}$ plot in a full PMT B scan. In this case, several points have been omitted using specific criteria concerning the $\chi$-square, the number of degrees of freedom and the mean value of each digitized spectrum. 


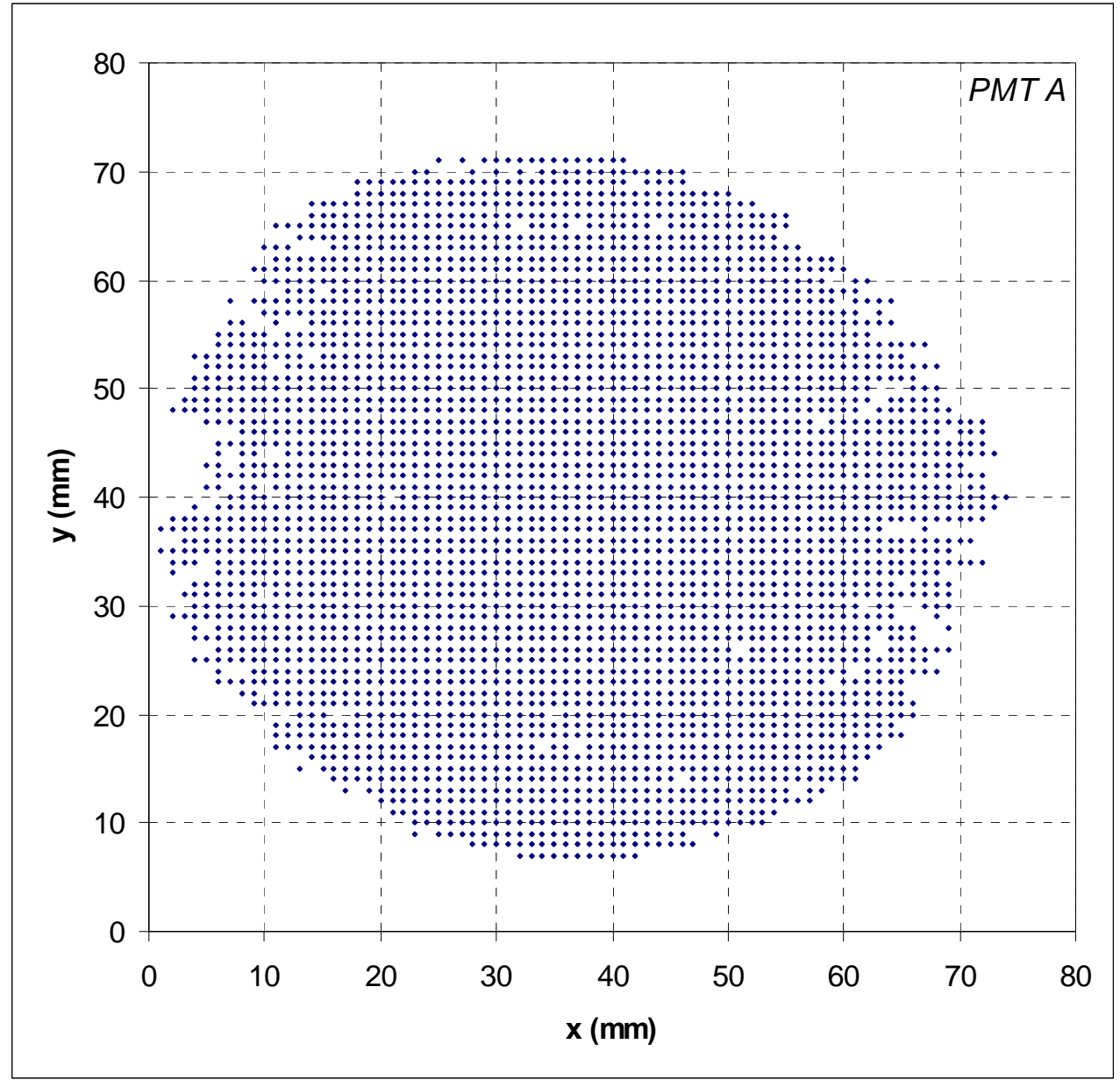

Figure 5.11: True positions $(\mathrm{x}, \mathrm{y})$ of the optical fiber derived by the calculated $(\mathrm{X}, \mathrm{Y})$ pairs for PMT A. 


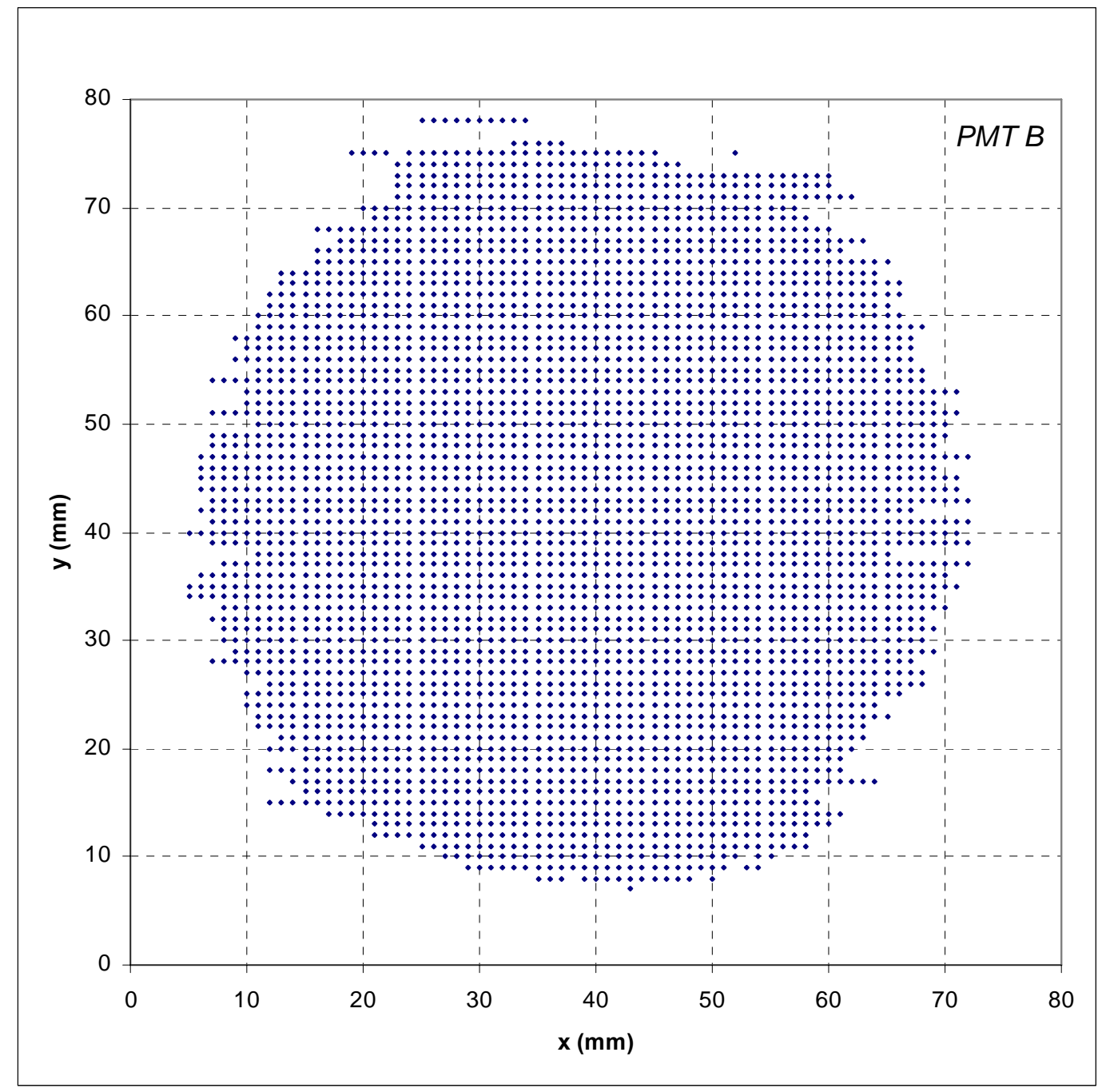

Figure 5.12: True positions (x,y) of the optical fiber derived by the calculated $(X, Y)$ pairs for PMT B.

\subsection{Derivation of $(\mathrm{x}, \mathrm{y})$ from $(\mathrm{X}, \mathrm{Y})$ pairs}

Observing the figures 5.8 and 5.10 someone can ask: "Is it possible to know the real position of the optical fiber $(\mathrm{x}, \mathrm{y})$ when only the values of the parameters $\mathrm{X}$ and $\mathrm{Y}$ are known?". We have answered that question and the arising problem with the use of an artificial neural network that we have developed in MATLAB. To train this program we have used half of the pairs $(\mathrm{x}, \mathrm{y})$ and $(\mathrm{X}, \mathrm{Y})$ to train the network and with the other half we have 
verified that the training is satisfactory. One example of the results of the training of the artificial neural network is given in figure 5.13.

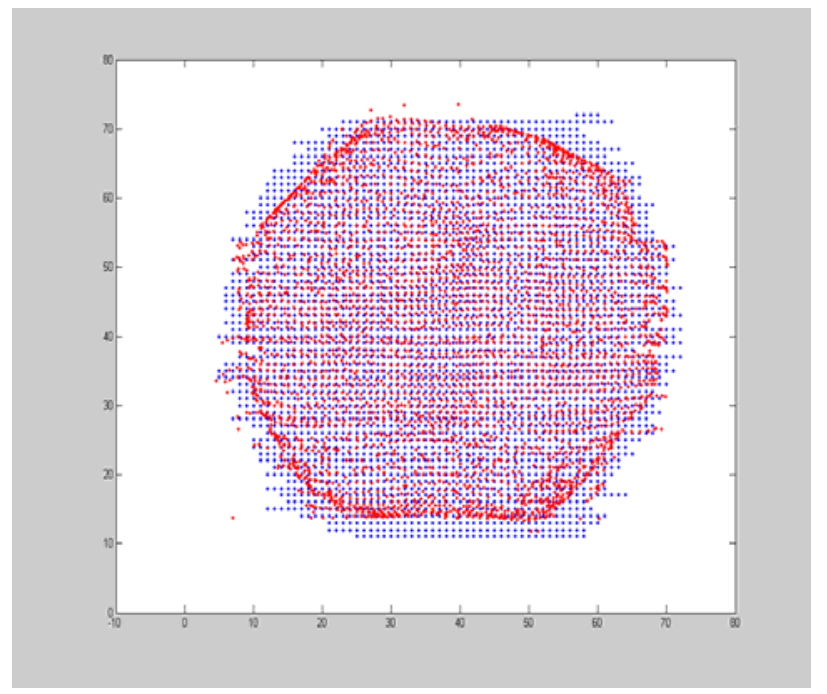

Figure 5.13: 2-dimensional image obtained before and after the application of a neural network on the data points $(\mathrm{x}, \mathrm{y})$. The vertical axis represents the values of the parameter $\mathrm{y}$, while the horizontal axis represents the values of the parameter $\mathrm{x}$. The blue points are the ones calculated by the digitized signals $\mathrm{XA}, \mathrm{XB}, \mathrm{YC}$ and $\mathrm{YD}$ with backprojection to the actual optical fiber position $(\mathrm{x}, \mathrm{y})$, while the red points correspond to the points $(\mathrm{x}, \mathrm{y})$ resulting from the trained neural network.

As it can be observed from figure 5.13, there is a very good approximation of the actual data points corresponding to the position of the optical fiber (x,y) with the data points resulting after training the neural network.

The more the neural network is trained, the better the accordance between the actual data points $(\mathrm{x}, \mathrm{y})$ and the output of the neural network.

\subsection{Edge Non-linearity Solution}

In order to achieve higher positioning accuracy, and avoid the problems shown in Figures 5.2 through 5.12, we have designed a different electronics system, where each of the 32 anode signals is processed individually [Tzanakos 2001]. A front end board having 33 amplifiers (32 anodes +1 dynode) is mounted on the PMT assembly to avoid noise pickup. 


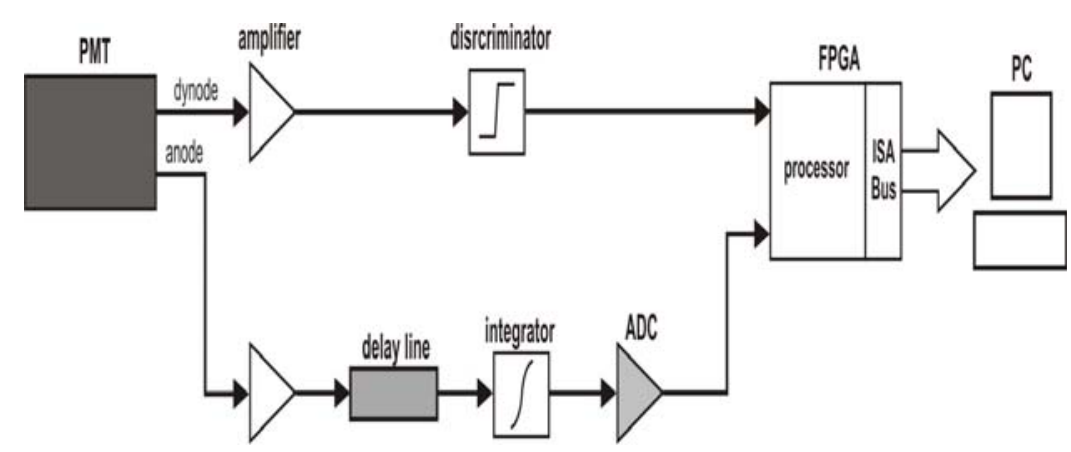

Figure 5.14: Electronics design (only one signal shown).

All anode signals after amplification, integration and digitization are processed by a Field Programmable Gate Array (FPGA), as shown in figure 5.14. Triggering, gating, and coincidence are achieved by using the dynode signals after discrimination. The 32 anode signals to the integrators must be delayed in order to be inside the integration gate. The digitized anode signals are used by the processor for the calculation of the real position of an event. The $(\mathrm{x}, \mathrm{y})$ values are transmitted to a PC for storage and further processing. The interface for the ISA Bus is fitted in the FPGA [Tzanakos 2001]. The processor is designed from scratch as a PLD (Programmable Logic Device) to cover exactly the demands of our system. The FPGA [FPGA] has been chosen to have adequate capacity and operate at high clock frequencies (several tens of $\mathrm{MHz}$ ) in order to handle the requirements of our system. The idea of using FPGAs for processing has been used by others in recent designs as well [Young 2000], [Moses 2001]. 


\section{Chapter 6}

\section{Conclusions}

We have developed a small animal PET scanner in order to study various design parameters on the imaging performance. Our measurements show that a number of parameters need to be studied and optimized at every stage of the scanner, in order to achieve the highest possible resolution at low noise levels and sufficient detection efficiency. The new idea here is the realization that a large number of signals could be processed individually using large scale integration and new devices like the FPGAs [Tzanakos 2001]. PMT performance can be further tuned by studying the necessary corrections to remove distortions in the position measurement. The apparent difficulty of how to reconstruct with circular PMTs is under study.

The choice of BGO over LSO was done for financial reasons. The crystal cross section size matches the anode wire pitch of the R2486 PMT. The crystal length of $20 \mathrm{~mm}$ was chosen for efficiency reasons although this will introduce depth of interaction effect on the resolution. Monte Carlo simulations [Pavlopoulos 1992, Tzanakos 1993] show that the resolution of the scanner at the center is $2 \mathrm{~mm}$ becoming $3.5 \mathrm{~mm}$ at $4 \mathrm{~cm}$ from the center for a FOV of $10 \mathrm{~cm}$ radius. Although this can be further improved, we think it is good enough for small animal imaging at this stage of development. It is conceivable that in the next step of our project we may choose smaller and shorter LSO crystals and PMTs with 
small pixels. One new feature of our design is that it allows the development of electronics to process independently the signal from the individual anodes [Tzanakos 2000, Tzanakos 2001]. This would mean that we will have to process 32 anode signals plus one dynode signal per PSPMT. Recent publications show that this trend is being considered by some authors [McElroy 2005].

Our next steps will focus on developing the electronics, constructing the platform on which system parts will function, and implementing software for 3D iterative image reconstruction. Measurements will be performed using specific phantom sources, in order to calibrate and prepare the system for actual laboratory measurements on small animals. 


\section{References}

Anger H.O. (1966), Survey of radioisotope cameras J. Nucl. Med. 5, 311-34

Bengel F.M., Ziegler S.I., Avril N., Weber W., Laubenbacher C. and Schwaiger M. (1997), Whole-body positron emission tomography in clinical oncology: comparison between attenuation-corrected and uncorrected images Eur. J. Nucl. Med. 24 1091-8

Bloomfield P. M., Myers R.,. Hume S.P et al. (1997), Phys. Med. Biol. 42389

Bohm C., Eriksson L., Bergstroem M., Litton J., Sundman R. and Singh M. (1978), A computer assisted ring detector positron camera system for reconstruction tomography of the brain IEEE Trans. Nucl. Sci. 25 624-37

Brownell G.L., Burnham C.A., Wilensky S., Arnonow S., Kaemi H. and Strieder D. (1969), New developments in positron scintigraphy and the application of cyclotron-produced positron emitter Medical Radioisotope Scintigraphy (IAEA Proceedings of a Symposium (Salzburg, Austria, August 1968)) (Vienna: IAEA) pp 163-76 
Bruyndoncky P. et al. (1997), NIM A 392407

Burnham C., Bradshaw J., Kaufman D., Chesler D. and Brownell G.L. (1983), A positron tomograph employing a one dimension BGO scintillation camera IEEE. Trans. Nucl. Sci. 30 661-4

Burnham C.A., Bradshaw J., Kaufmann D., Chesler D.A., Stearns C.W. and Brownell G.L. (1985), Design of a cylindrical shaped scintillation camera for positron tomographs IEEE Trans. Nucl. Sci. 32 889-93

CAEN Technical Information Manual, Mod. N471 2 Fold H.V. Power Supply, 12 Feb. 1993

Carson R.E., Daube-Witherspoon M.E. and Green M.V. (1988) A method of post-injection PET transmission measurements with a rotating source J. Nucl. Med. 29 1558-67

Chatziioannou A.F. et al. (1999), J. Nucl. Med. 401164

Chatziioannou A.F. (2002), Eur. J. Nucl. Med. 2998

Cherry S.R. et al. (1997), IEEE Trans. Nucl. Sci., NS-44 (3) 1161

Cho Z.H. and Farukhi M.R. (1977), Bismuth germanate as a potential scintillation detector in positron cameras J. Nucl. Med. 18 840-4

Colsher J.G. (1980), Fully three-dimensional positron emission tomography Phys. Med. Biol. 25 103-15

Del Guerra A. et al. (1998), IEEE Trans. Nucl. Sci. NS-45 (6) 3105

Derenzo S.E., Budinger T.F., Huesman R.H., Cahoon J.L. and Vuletich T. (1981), Imaging properties of a positron tomograph with 280 BGO crystals IEEE Trans. Nucl. Sci. 28 81-9 
FPGA The Spartan-3 Platform FPGA Family, Xilinx Inc., www.xilinx.com

Fries O. et al. (1997), NIM A 387220.

Hamamatsu "Photomultiplier tubes: Construction and operating characteristics"

Hamamatsu Position-Sensitive Photomultiplier tube type R2486 Technical Information.

Hamamatsu Position-Sensitive Photomultiplier Tubes with crossed wire anode Series R2486.

Herschman H.R. (2003), Curr. Opin. Immunol. 15378

Huber J.S. and Moses W.W. (1999), IEEE Trans. Nucl. Sci. NS-46 498

Jeavons A.P. et al. (1999), IEEE Trans. Nucl. Sci. NS-46 468

Jones T. (1996), The imaging science of positron emission tomography, Eur J Nucl Med 23: $807: 813$

Jorway www.jorway.com

Kenanova V. (2005), Cancer Res. 65622

Kuhl D.E., Edwards R.Q., Ricci A.R. and Reivich M. (1973), Quantitative section scanning using orthogonal tangent correction. J. Nucl. Med. 14 196-200

\section{LabVIEW www.ni.com}

LeCroy www.lecroy.com

McElroy D. P. et al. (2005), Phys. Med. Biol. 50 3323-3335 
Mechtronics http://www-esd.fnal.gov/esd/catalog/main/mechtron/150-spec.htm

Moses et al. (2001), IEEE Transactions on Nuclear Science NS-48, pp.632-636

Muehllehner G. and Wetzel R.A. (1971), Section imaging by computer calculation J. Nucl. Med. 12 76-85

Muehllehner G, BuchinMP and Dudek J H 1976 Performance parameters of a positron imaging camera IEEE Trans. Nucl. Sci. 23 528-37

Myers R. (2001), Nucl. Med. Biol. 28585

Myers R., Hume S. (2002), Eur. Neuropsychopharmacology 12545

Pavlopoulos S. and Tzanakos G. (1996), Conf. Record, IEEE NSS and MIC, San Fransisco, CA, Oct 31 - Nov. 6, 1993, pp. 1697-1701; IEEE Trans. Nucl. Sci. NS-43, 3249

Pavlopoulos S. (1992), PhD Thesis, Rutgers University, (unpublished).

Phelps M.E., Hoffman E.J., Mullani N.A., Higgins C.S. and Ter-Pogossian M.M. (1976), Design Considerations for a positron emission transaxial tomograph (PETT III) IEEE Trans. Nucl. Sci. 23 516-22

Phelps M.E. (2000), Positron Emission Tomography provides molecular imaging of biological processes. Proc Natl Acad Sci U S A 97: 9226-9233

Reivich M. et al. (1979), The [F-18]fluorodeoxuglucose method for the measurement of local cerebral glucose utilization in man Circ. Res. 44 127-37

Senda M. et al. (1985), Performance characteristics of Positologica III-a whole-body positron emission tomograph J. Comp. Assist Tomogr. 9 940-6 
Siegel S. et al., IEEE Trans. Nucl. Sci. NS-46 (1999) 571

Stickel J.R., Cherry S.R. (2005), Phys. Med. Biol. 50179

Stoecklin G. (1995), Fluorine-18 compounds Principles of Nuclear Medicine (Philadelphia: Saunders) pp 178-94

Sossi V., Ruth TJ. (2005), J Neural Transm. 112319

Tai Y.C., Laforest R. (2005), Annu. Rev. Biomed. Eng. 7255

Ter-Pogossian M.M., Mullani N.A., Hood J.T., Higgins C.S. and Ficke D.C. (1978), Design considerations for a positron emission transverse tomograph (PETT V) J. Comput. Assist. Tomogr. 2 539-44

Thompson C J, Yamamoto Y L and Myer E 1979 Positome II: a high efficiency positron imaging device for dynamic brain studies IEEE Trans. Nucl. Sci. 26 583-9

Tomaszewski P.E. (2002), J. Cryst. Growth 236, 1

Toyama H. (2005), Eur. J Nucl. Med. Mol. Imaging. Vol. 32, pp. 593

Tzanakos G. and Pavlopoulos S. (1993), Conf. Record, IEEE NSS and MIC, pp. 18421846; San Fransisco, CA, Oct 31 - Nov. 6

Tzanakos G., et al. (2000), Proc. IEEE 2000 Nucl. Sci. Symposium and Med. Imag. Conference, Lyon, 15-20 Oct 2000; 21_53

Tzanakos G., et al. (2001), Proc. IEEE 2001 Nucl. Sci. Symposium and Med. Imag. Conference, Oct. 2001, San Diego, California, Poster M9A-2 
Wang J., Maurer L. (2005), Curr. Top. Med. Chem. 51053

Watanabe M.et al. (1992), IEEE Trans. Med. Imag. 11:4, 577-580

Weber S. et al. (1999), IEEE Trans. Nucl. Sci. NS-46 1177

Weber S., Christ D., Kurzeja M. et al. (2003), Comparison of LuYAP, LSO, and BGO as Scintillators for High Resolution PET Detectors. IEEE Trans Nucl Sci 50: 1370-1372

Wong W H, Mullani N A, Philippe E A, Hartz R K, Bristow D, Yerian K, Gaeta J M and Ketharnavaz N 1984 Performance characteristics of the University of Texas TOFPET-I PET camera J. Nucl. Med. 25 46-7

Young et al. (2000), IEEE Trans. Nucl. Sci. 47(4), 1676-1680 
